\title{
WING-WING INTERACTIONS IN ROTATING LOCUST WING MODELS
}

\author{
by
}

\author{
Jennifer J. Fauquier \\ B.Sc., Carleton University, 2008
}

A thesis submitted to the Faculty of Graduate Studies and Postdoctoral Affairs in partial fulfillment of the requirements for the degree of

Master of Science

In

Biology

Ottawa-Carleton Institute of Biology

Carleton University

Ottawa, Ontario

(C) 2011, Jennifer J. Fauquier 


$\begin{array}{ll}\begin{array}{l}\text { Library and Archives } \\ \text { Canada }\end{array} & \begin{array}{l}\text { Bibliotheque et } \\ \text { Archives Canada }\end{array} \\ \begin{array}{l}\text { Published Heritage } \\ \text { Branch }\end{array} & \begin{array}{l}\text { Direction du } \\ \text { Patrimoine de l'édition }\end{array} \\ 395 \text { Wellington Street } & 395, \text { rue Wellington } \\ \text { Ottawa ON K1A ON4 } & \text { Ottawa ON K1A ON4 } \\ \text { Canada } & \text { Canada }\end{array}$

Your file Votre référence
ISBN: $978-0-494-81694-3$
Our file Notre référence
ISBN: $978-0-494-81694-3$

NOTICE:

AVIS:

The author has granted a nonexclusive license allowing Library and Archives Canada to reproduce, publish, archive, preserve, conserve, communicate to the public by telecommunication or on the Internet, loan, distribute and sell theses worldwide, for commercial or noncommercial purposes, in microform, paper, electronic and/or any other formats.

The author retains copyright ownership and moral rights in this thesis. Neither the thesis nor substantial extracts from it may be printed or otherwise reproduced without the author's permission.

L'auteur a accordé une licence non exclusive permettant à la Bibliothèque et Archives Canada de reproduire, publier, archiver, sauvegarder, conserver, transmettre au public par télécommunication ou par l'Internet, prêter, distribuer et vendre des thèses partout dans le monde, à des fins commerciales ou autres, sur support microforme, papier, électronique et/ou autres formats.

L'auteur conserve la propriété du droit d'auteur et des droits moraux qui protège cette thèse. Ni la thèse ni des extraits substantiels de celle-ci ne doivent être imprimés ou autrement reproduits sans son autorisation.
In compliance with the Canadian Privacy Act some supporting forms may have been removed from this thesis.

While these forms may be included in the document page count, their removal does not represent any loss of content from the thesis.
Conformément à la loi canadienne sur la protection de la vie privée, quelques formulaires secondaires ont été enlevés de cette thèse.

Bien que ces formulaires aient inclus dans la pagination, il n'y aura aucun contenu manquant.

\section{Canadä}




\section{Abstract}

The complex, non-linear motions of flapping insect wings, coupled with their small size and mass, mean that insects do not generate lift via the same aerodynamic mechanisms used by large birds and man-made aircraft. The majority of studies into the aerodynamic mechanisms of lift production by insects have employed insect species that are functionally two winged and thus the complex nature of the interaction of wing couples, specifically forewing-hindwing interactions, has been largely unexplored. The objective of this thesis was to characterize, using high-speed digital cinematography, dye flow visualization of model wings and volumetric 3-component digital particle image velocimetry, the interaction between forewing-hindwing couples during straight flight and during steering in locusts; a functionally four-winged insect that flies with a steeply inclined stroke plane. Kinematic analyses determined biologically relevant values of key kinematic variables defining the position of forewings and hindwings during the downstroke. The fluid structure over the hindwing, including the presence of a hindwing leading edge vortex, was found to be very sensitive to the presence of a forewing at moderate and high angles of attack. The separation angle between forewing and hindwing was not as important as the difference in elevation angles of the forewing and hindwing during the downstroke. In general, wing-wing interactions result in the abolishment of a LEV on the hindwing by preventing separation at the leading edge. It is hypothesized that this results in a decrease in lift produced by the wing couple. Kinematic observations during steering attempts are consistent with this strategy to decrease lift produced by the wing couple on the inside of the turn path. 


\section{Acknowledgments}

First and foremost, I'd like to thank Dr. Jeff Dawson for his guidance and advice through my years of research in insect flight at Carleton University; it has been a privilege to work with you. I'd also like to thank Em Standen for her help and advice throughout this journey. A special thanks to all my lab mates, Amanda, Ryan and Sarah for their continuous support during my time in the Dawson lab. Thank you to Mike Jutting for all of his technical assistance with our custom made equipment.

I'd like to express my thanks and appreciation to the late Dr. James Fullard for the use of his equipment throughout this study and also to Dr. Steven Cooke for the use of his camera.

I'd like to express my appreciation to NSERC and CFI for providing grants and funding to our lab so that research like this is possible.

I'd also like to express my gratitude to the department of Biology for awarding me with an internal scholarship and bursaries that have allowed me to continue with my studies.

On a personal note, I'd like to thank my parents, brother, sister-in law and my husband's family for their continuous support throughout my education. Carol Anne Austin, thank you for being my best friend, confidant and partner in crime. Finally, I'd like to thank my husband, Mathew, you have been there through thick and thin and have supported me and I will always be grateful I have someone like you who will always be there for me. 


\section{Table of Contents}

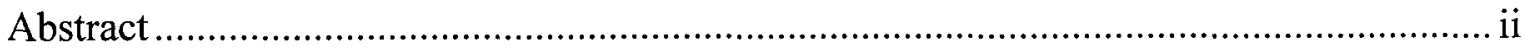

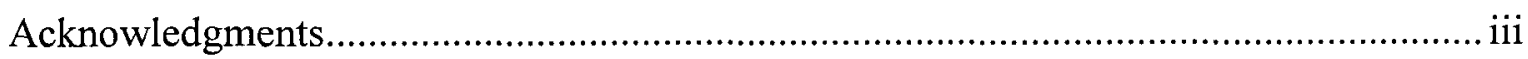

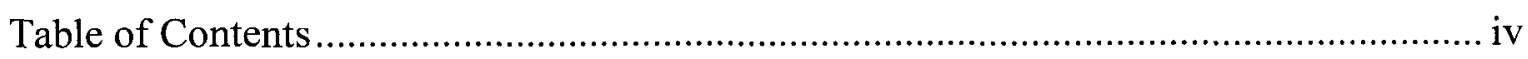

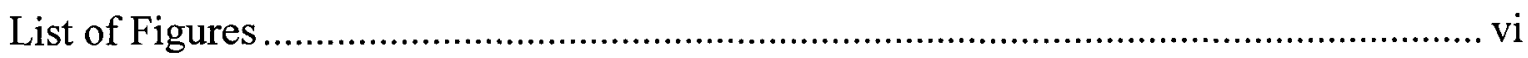

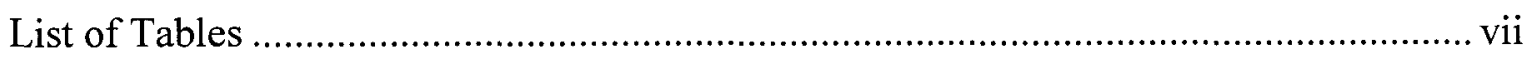

List of Abbreviations and Symbols........................................................................ viii

Chapter 1: General Introduction and Literature Review................................................ 1

1.1 Basic Kinematics and Aerodynamics ............................................................ 3

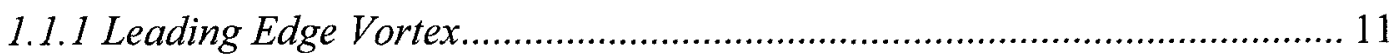

1.1.2 The Clap-Fling Mechanism ................................................................ 13

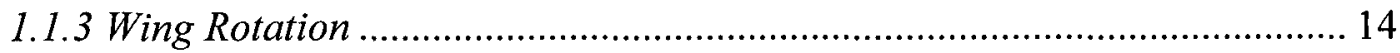

1.1.5 Wing-Wing Interactions ........................................................................ 15

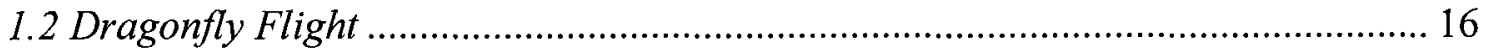

1.3 Methods for Studying Insect Flight ............................................................... 17

1.4 Flapping Models versus Rotating Models ......................................................... 19

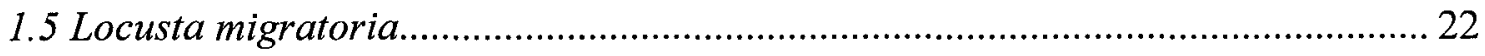

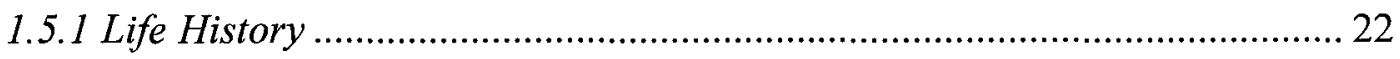

1.5.2 Using Locusts to Study Flight ............................................................... 22

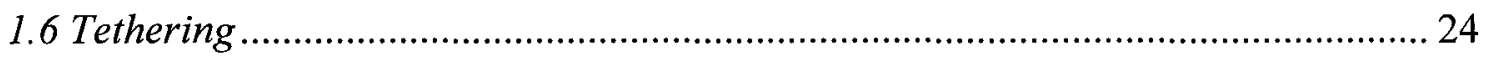

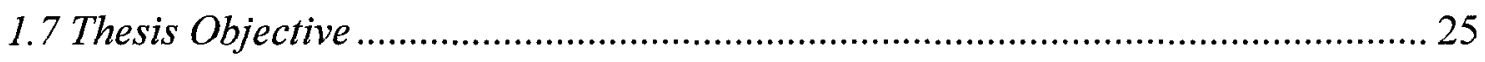

Chapter 2: Wing-Wing Interactions in Rotating Locust Wings....................................... 27

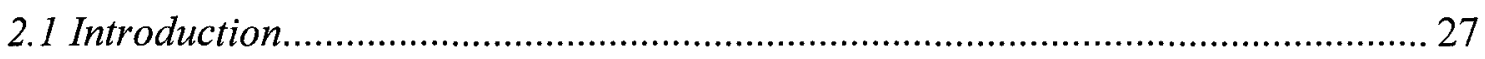

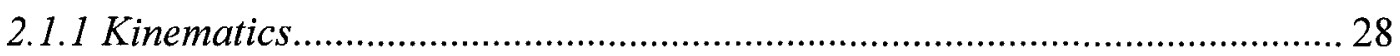

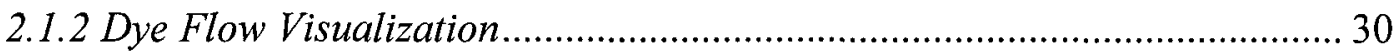

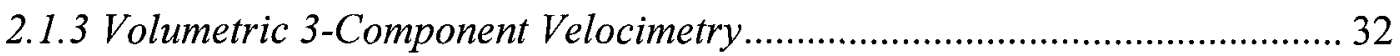

2.1.4 Rotating Wing Models............................................................................... 33

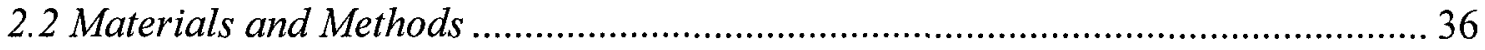

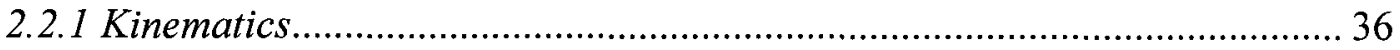

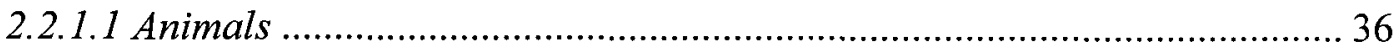

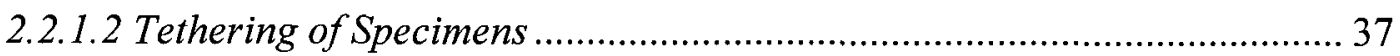

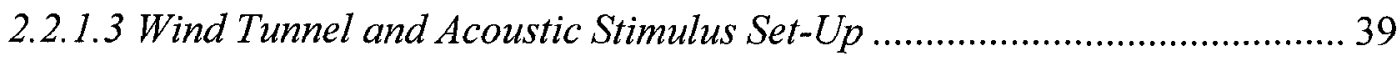

2.2.1.4 High-Speed Stereo Digital Cinematography ............................................. 43

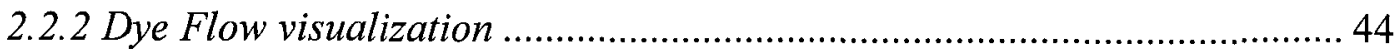


2.2.2.1 The Spinner Tank 44

2.2.2.2 Wing Models 48

2.2.3 Volumetric 3-Component Velocimetry. 51

2.2.3.1 System Calibration and Tank Set-Up. 51

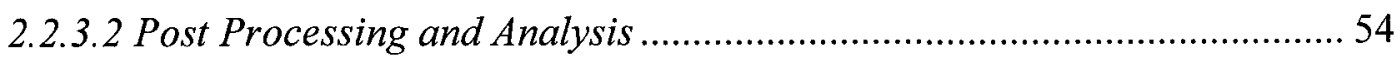

2.3 Results. .55

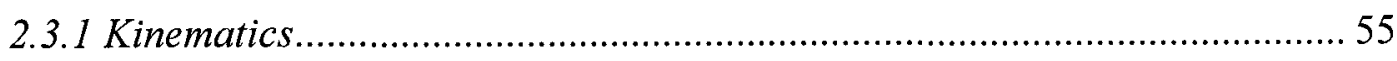

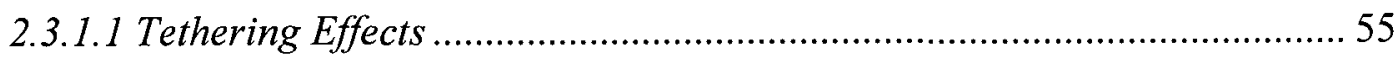

2.3.1.2 Steering (Pre vs. Post-Stimulus) .......................................................... 56

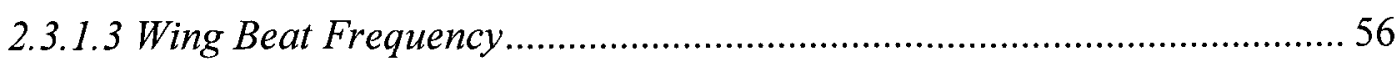

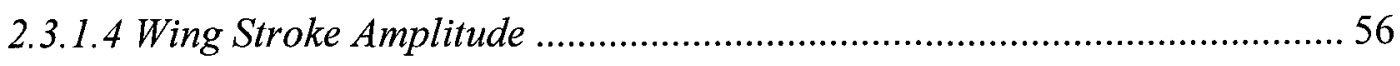

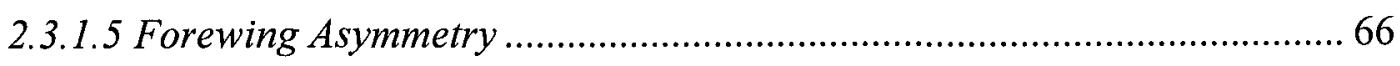

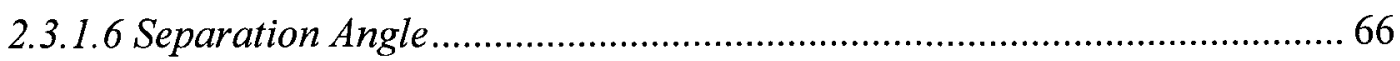

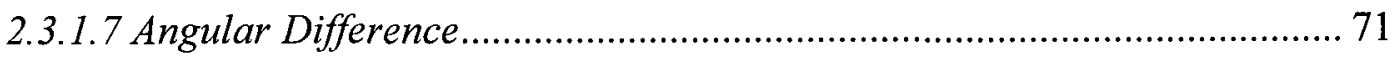

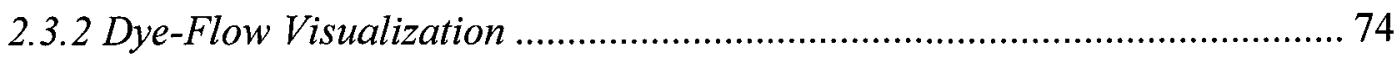

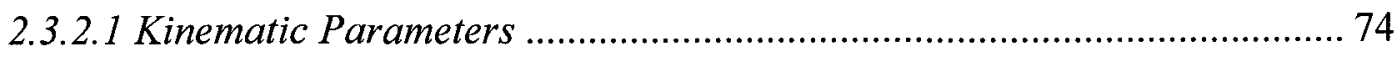

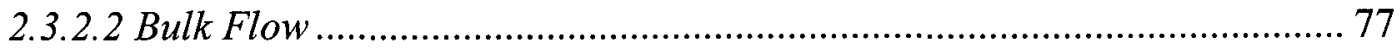

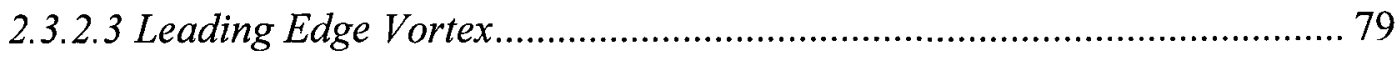

2.3.3 Volumetric 3-Component Velocimetry ......................................................... 91

2.3.3.1 Qualitative Analysis of Flow Structures ................................................... 91

2.3.3.2 Velocity Magnitude of the LEV Structures.............................................. 96

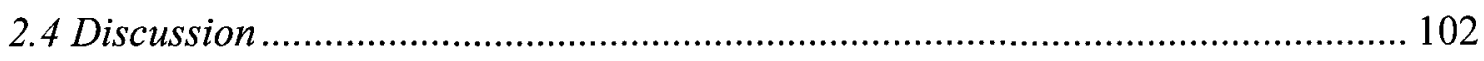

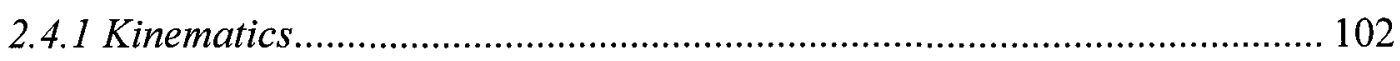

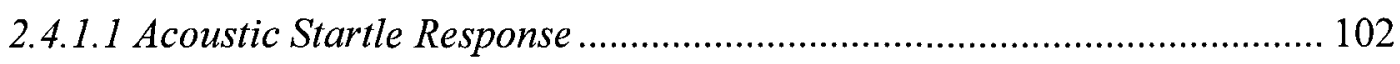

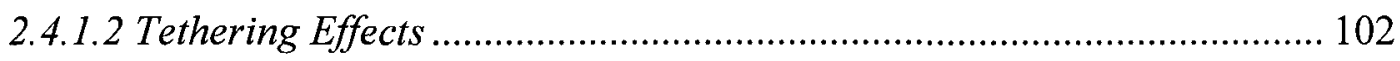

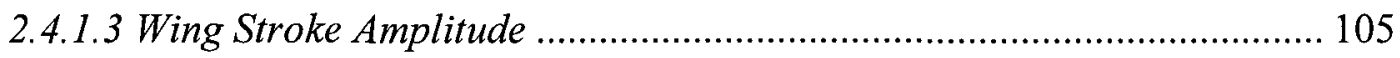

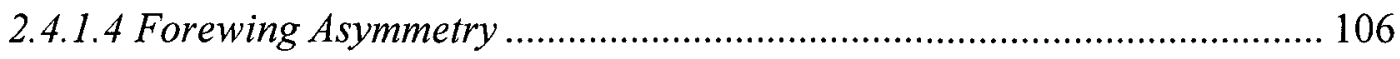

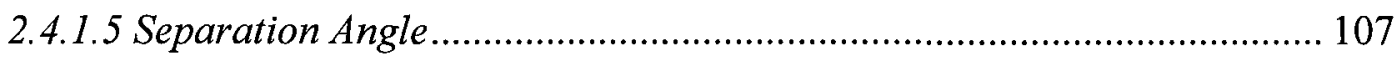

2.4.1.6 Angular Difference ............................................................................... 107

2.4.2 Wing-Wing Interactions in Rotating Wing Models .................................... 110

2.4.2.1 Overall Bulk Flow........................................................................... 110

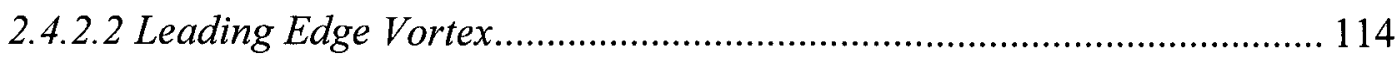

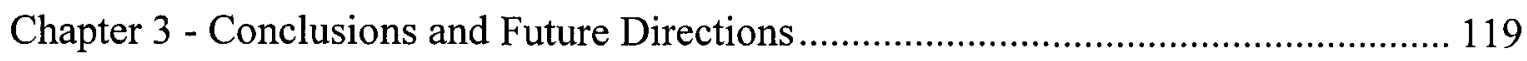

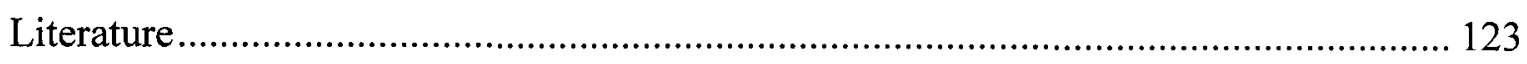




\section{List of Figures}

Figure 1.1

Figure 1.2

Figure 1.3

Figure 1.4

Figure 1.5

Figure 1.6

Figure 1.7

Figure 2.1

Figure 2.2

Figure 2.3

Figure 2.4

Figure 2.5

Figure 2.6

Figure 2.7

Figure 2.8

Figure 2.9

Figure 2.10

Figure 2.11

Figure 2.12

Figure 2.13

Figure 2.14

Figure 2.15

Figure 2.16

Figure 2.17

Figure 2.18

Figure 2.19

Figure 2.20

Figure 2.21

Figure 2.22

Figure 2.23

Figure 2.24

Figure 2.25

Figure 2.26

Figure 2.27

Figure 2.28

Figure 2.29

Figure 2.30

Figure 2.31

Figure 2.32

Figure 2.33

Figure 2.34

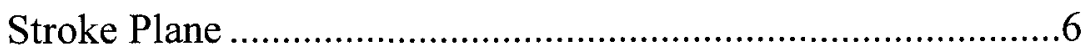

Angle of Attack .....................................................................

Wing Stroke Amplitude ..........................................................

Angular Difference ................................................................

Forewing Asymmetry ............................................................10

Separation Angle ……………………………………......12

Chord-Wise Flow Over Wings ………………………............21

Perspective Issue in Kinematic Analyses ................................29

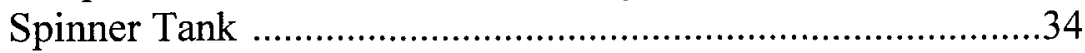

Tethering Methods .................................................................40

Wind Tunnel ........................................................................

Calibration Grid .....................................................................

Digitized Points on a Locust ......................................................46

Kinematic Results of a Real Flying Locust ..............................47

Wing Model Configuration Measurements ..............................50

Tank Used for V3V .................................................................

Wing Beat Frequency Results..................................................58

Wing Stroke Amplitude of the Ipsilateral Forewings ..............62

Wing Stroke Amplitude of the Contralateral Forewings .........63

Wing Stroke Amplitude of the Ipsilateral Hindwings .............64

Wing Stroke Amplitude of the Contralateral Hindwings ........65

Forewing Asymmetry of Left Turning Locusts ........................67

Forewing Asymmetry of Right Turning Locusts.......................68

Separation Angles of the Ipsilateral Wing Pairs ......................69

Separation Angles of the Contralateral Wing Pairs ...................70

Angular Differences of the Ipsilateral Wing Pairs....................75

Angular Differences of the Contralateral Wing Pairs...............76

Bulk Flow of a Locust Wing Pair ...........................................78

Dye-Flow Visualization of Locust Hindwings in Isolation .....80

Dye-Flow Visualization of a Rotating Wing Couple I ............87

Dye-Flow Visualization of a Rotating Wing Couple II ............89

Dye-Flow Visualization of a Rotating Wing Couple III...........90

Vorticity Isocontours of a Rotating Wing Couple I.................92

Vorticity Isocontours of a Rotating Wing Couple II ................93

Vorticity Isocontours of a Rotating Wing Couple III ..............94

Vorticity Contour of a Rotating Wing Couple...........................95

Flow Field Around a Rotating Wing Couple I..........................98

Flow Field Around a Rotating Wing Couple II ........................99

Flow Field Around a Rotating Wing Couple III .....................100

Flow Field Around a Rotating Wing Couple IV ......................101

Wingtip Path of a Locust .......................................................109 


\section{List of Tables}

Table 2.1 Locust Morphometrics, Stimulus and Wind Tunnel

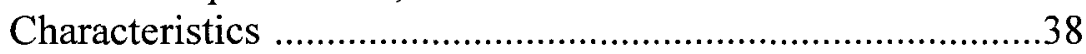

Table 2.2

Wing Beat Frequencies and Forewing Asymmetries

Table 2.3

Wing Stroke Amplitudes .60

Table 2.4 Separation Angles and Angular Differences ............................72

Table 2.5

Table 2.6

Detachment Points of Hindwings in Isolation

Table 2.7

Summary of Dye-Flow Visualization Results . .83

Summary of Velocity Magnitudes 


\section{List of Abbreviations and Symbols}

\begin{tabular}{ll} 
V3V & Volumetric 3-Component Velocimetry \\
dPIV & Digital Particle Image Velocimetry \\
WSA $(\Phi)$ & Wing Stroke Amplitude \\
AD $(\delta)$ & Angular Difference \\
SA $(\theta)$ & Separation Angle \\
FWA $(\gamma)$ & Forewing Asymmetry \\
ASR & Acoustic Startle Response \\
Ipsi & Ipsilateral Wing, the Wing on the Inside of the Turn Path \\
Contra & Contralateral Wing, the Wing on the Outside of the Turn Path \\
$\alpha$ & Geometric Angle of Attack \\
$\beta$ & Stroke Plane \\
$\chi$ & Body Angle \\
Re & Reynolds Number \\
LEV & Leading Edge Vortex \\
Dp & Detachment Point \\
$1^{\circ}$ & Primary LEV Structure \\
$A R$ & Aspect Ratio \\
$\mathrm{R}$ & Wing Length \\
$\mathrm{c}$ & Cord Length of the Wing \\
$v$ & Kinematic Viscosity of Air at $30^{\circ} \mathrm{C}$ \\
$\mathrm{v}$ & Velocity of Air (Depending on Equation) \\
$\mathrm{f}$ & Frequency \\
$\rho$ & Density of Air at $30^{\circ} \mathrm{C}$ \\
$\mu$ & Dynamic Viscosity of Air or Water at $30^{\circ} \mathrm{C}$ \\
$s$ & Span of the Airfoil \\
$\mathrm{v}$ & Velocity of the Object \\
$\Gamma$ & Circulation \\
\hline &
\end{tabular}




\section{Chapter 1: General Introduction and Literature Review}

“...diverse features of insect biology are united by the commonality of biomechanical performance during flight..."

'The Biomechanics of Insect Flight'

Robert Dudley, 2000

All insects need to overcome two challenges in order to fly: they need to produce enough lift to overcome their own weight and to produce sufficient thrust to compensate for the drag forces that result as a consequence of body shape and wing movement during forward flight.

Insect flight is arguably the most significant and beneficial adaptation in insect evolution. Until recently, flight in many insects could not be studied in great detail because the technology was not available to view and measure the complex motions of the wings and body. Research into insect flight has intensified greatly since 1997 owing to the use of high-speed cinematography, bio-inspired robotic models, improvements in flow visualization techniques and increased performance of microcomputers for computational models of fluid dynamics. These advances have clarified and directly led to the discovery of four unsteady mechanisms suggested to aid in the production of forces for flapping flight: the leading-edge vortex/dynamic stall (LEV), wake recapture, rotational lift and the clap -fling mechanism. These mechanisms are fundamentally different than those conventional (steady) mechanisms at play in large birds and airplanes. These four mechanisms along with other kinematic characteristics such as stroke plane, wing-wing interactions and overall body shape and wing camber are the focus of studies that attempt to establish the basis of aerodynamics in insect flight. 
To date, the majority of the research on insect flight has been conducted on relatively few model species, for example Drosophila (fruitflies), Manduca (hawkmoth), and a few species of hymenoptera (bees and wasps e.g. Apis, sp. and Encarsia sp.), all of which are functionally two winged, hovering species. More recently some attention has turned to dragonflies (Odonata), which are highly maneuverable, four-winged insects also capable of hovering. Much of these data have been assumed to be applicable to two and four-winged insects alike (Usherwood and Lehmann, 2008). Given the specialist nature of these species ecology, many of these insects possess unique wing and body morphologies and kinematic characteristics during flight emphasizing the importance of considering other insect species, especially those from insect orders other than the three noted above. Studying representatives from different insect orders would result in more accurate descriptions of the aerodynamics of insect flight in general, and will reveal the governing principles of flapping wing flight aerodynamics.

Locusts are functionally four-winged insects wherein the forewings are not mechanically coupled to the hindwings with structures such as jugal folds (Lepidoptera) or hamuli (hymenoptera). During forward flight, the phase relationship between forewings and hindwings suggests that significant wing-wing interactions may occur. Locusts beat their wings within a steeply inclined (almost vertical) stroke plane and behavioural observations of locusts show they are incapable of true hovering but are able to fly along steeply inclined trajectories. These observations are consistent with their wing morphology, wing beat frequency and wing kinematics, and given the differences in wing morphology and stroke plane characteristics seen in flies, bees, moths and dragonflies, these differences likely represent important, fundamental, differences in 
flight mechanics.

The purpose of this chapter is to provide a framework by reviewing the literature associated with insect flight, and more specifically, wing-wing interactions. In this review, I will start with a brief description of the kinematics and aerodynamics of a typical insect and discuss the principle high-lift generating aerodynamic mechanisms that insects use for generating lift and thrust. I will then discuss the limitations of current studies of insect flight. Functionally four-winged insects, such as locusts - the model chosen for this study, pose a particular challenge in understanding insect flight because of the unknown mechanisms governing wing-wing interactions; therefore, I will discuss the limited literature available on wing-wing interactions and the hypotheses associated with those studies. My review will then address dragonfly flight as they are one of the most widely studied functionally four-winged insects, and how their flight kinematics differ from locusts. Next, I will discuss methods used to study insect flight such as dye-flow techniques, volumetric 3-component velocimetry (V3V) system and mechanical models as well as flapping and rotating wing models. I will also discuss Locusta migratoria in terms of its life history and using locusts to study flight. Next, I will discuss tethering, a key method that is almost always employed when studying insect flight. I will conclude this chapter by stating my overall thesis objective.

\subsection{Basic Kinematics and Aerodynamics}

In order to achieve flight, insects and aircraft alike need to generate sufficient lift and thrust to overcome the forces resulting from its weight (gravity) and shape (drag). 
Induced drag is an unavoidable by-product of lift and runs parallel to the direction of flow. The overall drag of an object is called the dynamic drag and in insects and airplanes, is the result of two kinds of drag, pressure drag and viscous drag. Pressure drag is the result of the surface area and surface features of an object having to 'push' the air out of the way stream causing a disturbance in flow pattern and resulting in turbulent flow structures and a force impeding on the object in the opposite direction of flow. Insects with a greater surface area perpendicular to the flow, such as bees, would experience more pressure drag than a more streamlined insect with a relatively small surface area perpendicular to the flow (i.e. locusts). Viscous drag is the direct result of fluid particles encountering the surface of the object and moving at a different velocity and direction than the flow stream particles. In contrast, a fluid flowing past the surface of a body exerts a force on it perpendicular to the direction of flow, this is called lift. At low velocities, higher angles of attack are required to generate enough lift to overcome drag, where as in high velocities, lower angles of attack are sufficient to maintain steady lift forces (Vogel, 2003).

In aircrafts, everything remains in a fixed position; for example the wings are set in a fixed position relative to the body of the aircraft and to each other and also maintain a given angle of attack (aside from flaps that are released during take-off and landing to reduce stalling speeds so the aircraft can safely flying at lower velocities). Aircrafts exhibit a cambered wing in which air must travel faster over the top of the wing to meet the slower air flowing under the wing, this is known as the Kutta condition. This results in a low pressure area above the wing and a higher pressure under the wing producing lift. The higher the velocity of the aircraft, the greater the pressure differences will be 
thus resulting in greater lift production. Aircrafts follow what is termed conventional (steady state) aerodynamics (Vogel, 2003).

In contrast to conventional (steady state) aerodynamics, flapping wings produce unsteady flows and thus cannot employ the same aerodynamic principles that large aircrafts (and birds) rely upon. Wing strokes of insects are comprised of two phases: the translational phase (the downstroke and upstroke of the wings), and the rotational phase (occurring at the stroke reversal, where the wing rapidly pronates or supinates for the subsequent half-stroke). During these half strokes, the wings accelerate from an initially stationary position (at the moment of the stroke reversal) to a peak velocity, then decelerate rapidly until the next stroke reversal. The wing movement (flapping) occurs in a stroke plane, which is defined by the wing tip path through the air measured relative to the horizontal (or body angle) of the insect (Figure 1.1). At the stroke reversal, the wings must rotate to establish the correct angle of attack for the subsequent half-stroke. The angle of attack is not fixed during the half stroke (i.e. downstroke), but changes throughout the stroke presumably to maximize lift (or to effect a steering torque) (Figure 1.2). Wing stroke amplitude (WSA) (Figure 1.3) and wing beat frequency (WBF) are also modulated during flight in complex ways. Relatively recently, asymmetric forewing depression (FWA), has been shown to correlate with attempted steering direction in tethered, flying locusts (Dawson, et al, 1997) (Figure 1.5). Coincident with FWA is the production of angular differences (AD) and changes in the separation angle of forehindwing couples which may be important for steering torque production. Angular difference is defined roughly as the 'gap' observed between a locust forewing and hindwing during the downstroke when the insect if viewed from behind (Figure 1.4). 

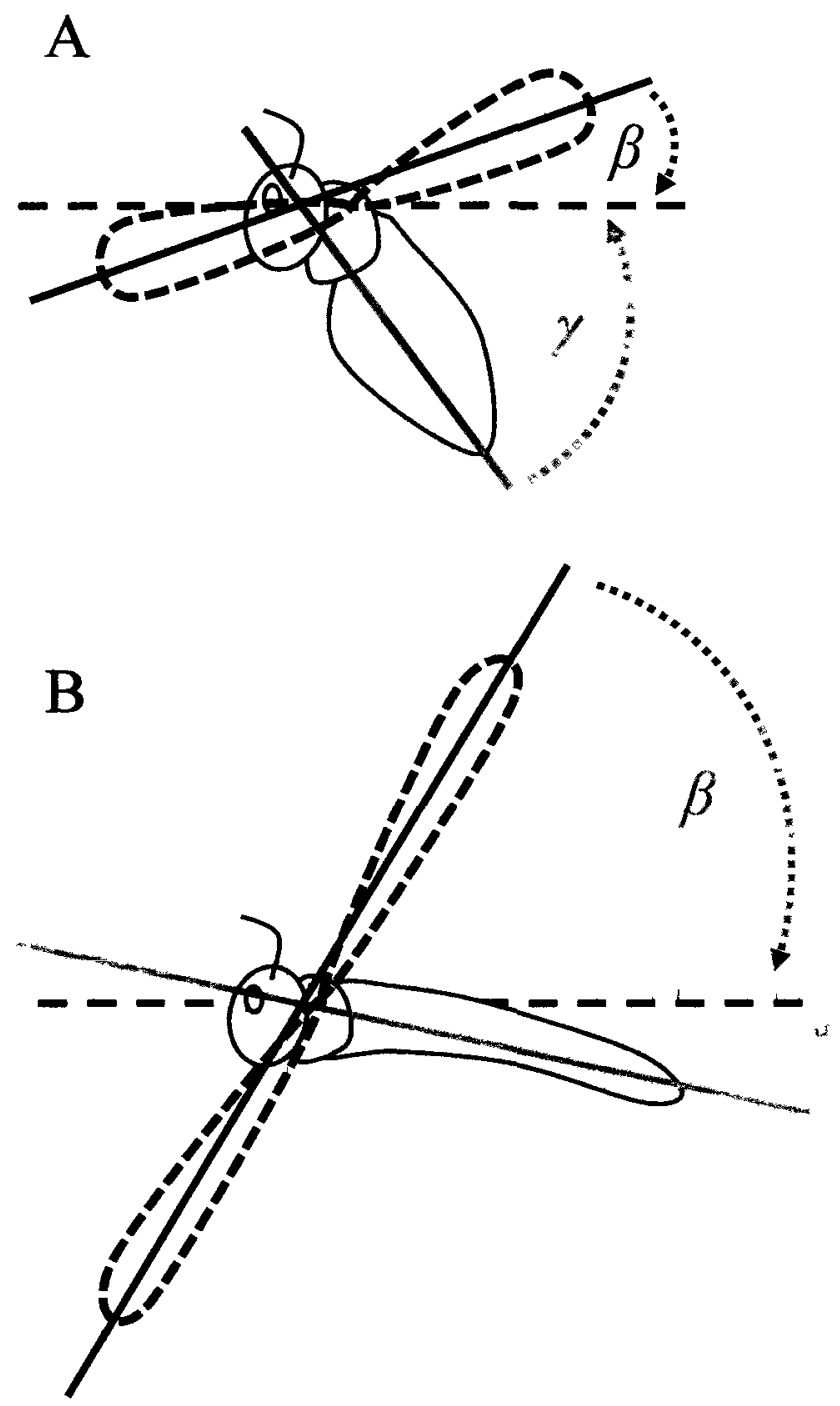

Figure 1.1. Schematic diagram illustrating the stroke plane $(\beta)$ and body angle $(\chi)$ for a fly (A) and a locust (B). The stroke plane is defined as the angle of the mean wing tip path relative to the horizontal. The fly typically exhibits an almost horizontal stroke plane during hover. In contrast, locusts fly with a steeply inclined, almost vertical stroke plane. The black dashed lines represent the path of the wing tip during forward flight. Note, only the forewings are shown for the locust for clarity. 
A

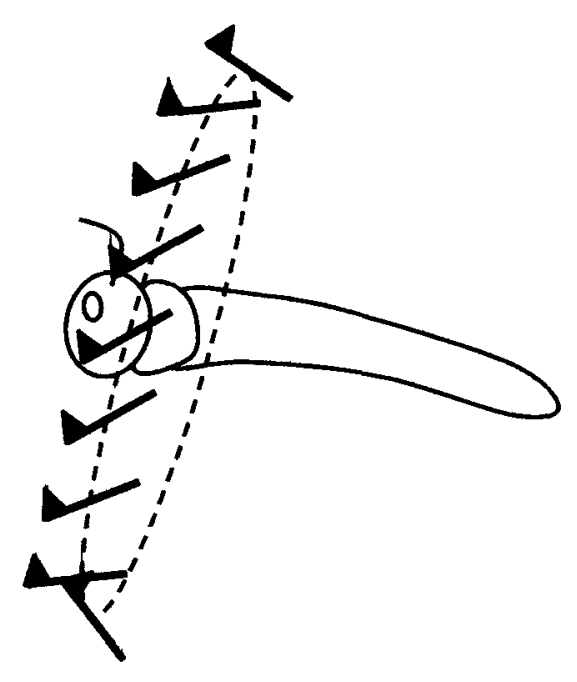

B

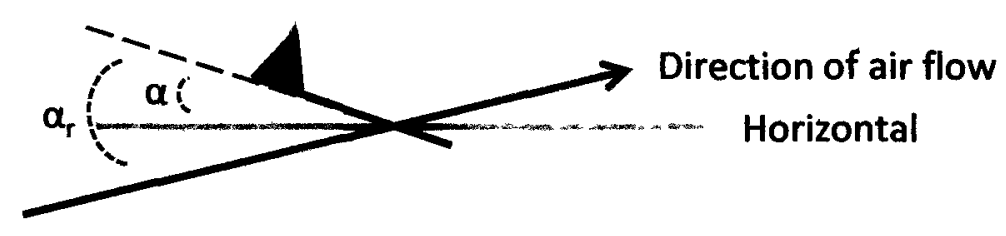

Figure 1.2. Schematic illustration of the forewing angles of attack during the downstroke (A). Dotted line is the wing tip path. The forewing is represented by a line (chord) with a triangle at the leading edge indicating the dorsal surface. The geometric angle of attack $(\alpha)$ of the wing is the angle formed by the wing chord relative to the horizontal. In contrast, the aerodynamic angle of attack $\left(\alpha_{\mathrm{r}}\right)$ is the angle of the wing chord relative to the air flow. 
A

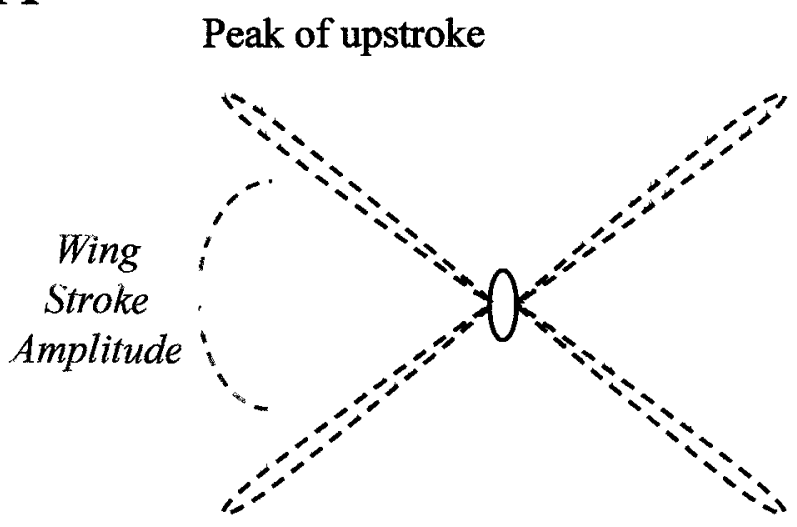

Peak of downstroke

Figure 1.3. Schematic illustration showing wing stroke amplitude (WSA) of a locust viewed from behind. Dashed outlines represent the wing in the upstroke and downstroke position. Amplitude is calculated as the difference is maximum upstroke angle minus maximum downstroke angle. 


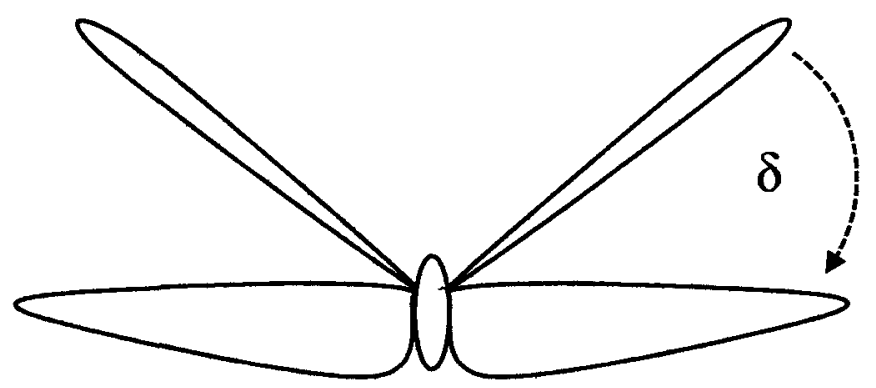

Figure 1.4. Schematic representation of a locust (posterior view) showing angular difference, also known as gap angle, $(\delta)$. Angular difference is defined as the difference in elevation angle of the forewing and hindwing at $1 / 3^{\text {rd }}$ the downstroke. 
A
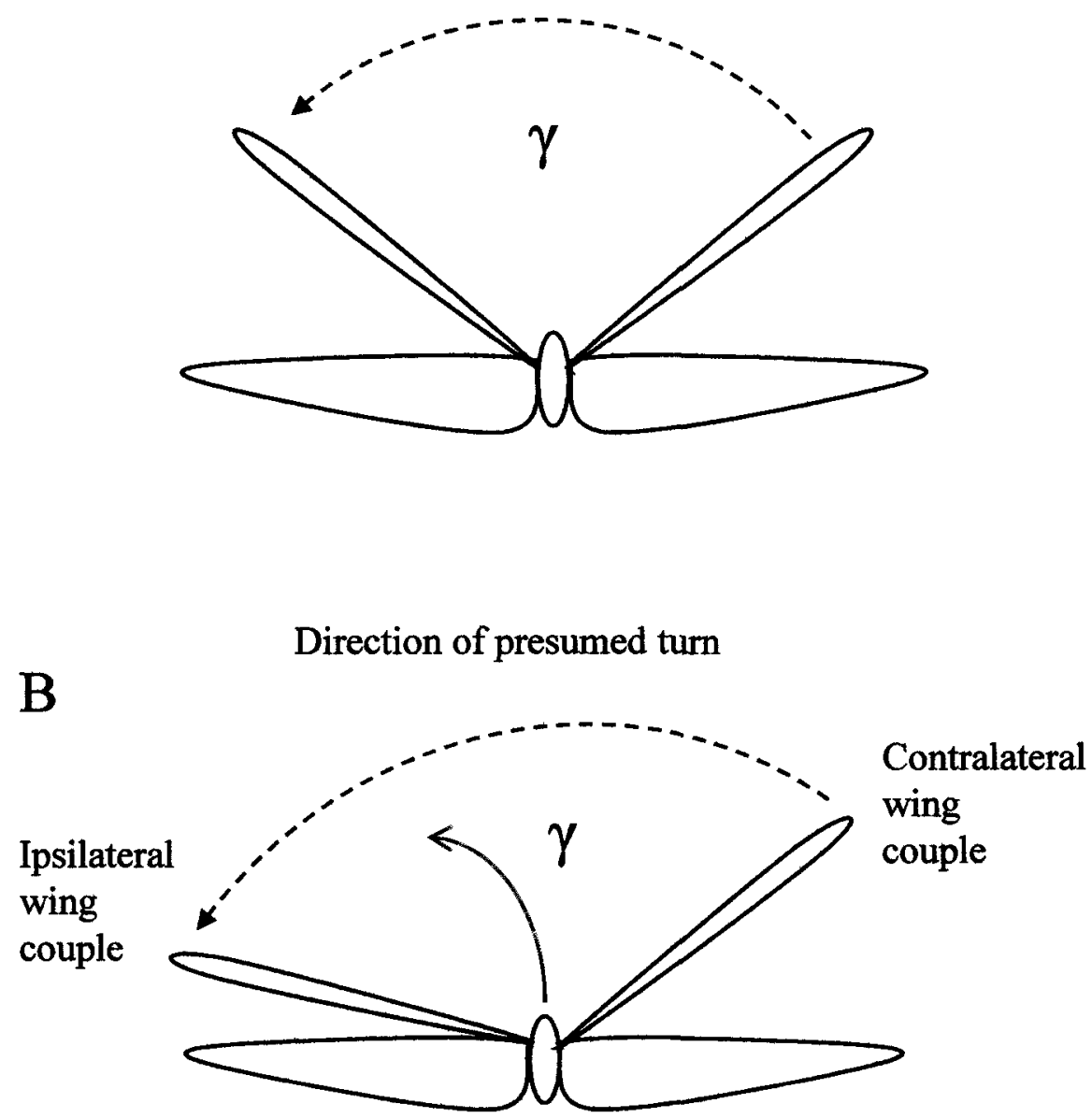

Figure 1.5. Diagram depicting forewing asymmetry $(\gamma)$ prior to a stimulus (A) and after a stimulus inducing the locust to turn to the left (B). After the stimulus, the FWA increases and the angular difference of the ipsilateral wing couple is less than angular difference of the contralateral wing couple. 
Separation angle (SA) is defined as the angle formed between forewing and hindwing when the insect is viewed from above (Figure 1.6).

Intensive research since the mid 1990's has resulted in the characterization of three high-lift generating mechanisms used by insects during flight: The leading edge vortex, rotational lift and wake capture. A fourth mechanism, called clap-fling (Weis-Fogh, 1973 ) is largely a special case of the LEV.

\subsubsection{Leading Edge Vortex}

Leading edge vortex (LEV) formation is the most important unsteady mechanism responsible in the generation of high-lift. It was first described by Ellington et. al. in 1996. A leading-edge vortex is formed at the base of the wings at the beginning of a downstroke. It is created when air meets the sharp leading edge of the insect wing and separates creating a vortex or separation bubble. The vortex remains attached to the surface of the wing where it spirals out to the wing tip via spanwise flow at a velocity relative to the wing tip velocity and forward flight velocity. In some cases, the vortex is shed at the stroke reversal when the wing has a velocity of zero (the transition of the downstroke to the upstroke and vice-versa), in other cases, it is shed continuously at the wing tip as a tip vortex throughout the stroke. Insects that fly with wings beating in a horizontal stroke plane and that are able to rotate the wing at the stroke reversal (flies, bees, and dragonflies for example) are able to create LEV's during both the downstroke and the upstroke (van den Berg \& Ellington, 1997). The angle of attack of the wing determines the size and magnitude of the LEV; as the angle of attack increases the 


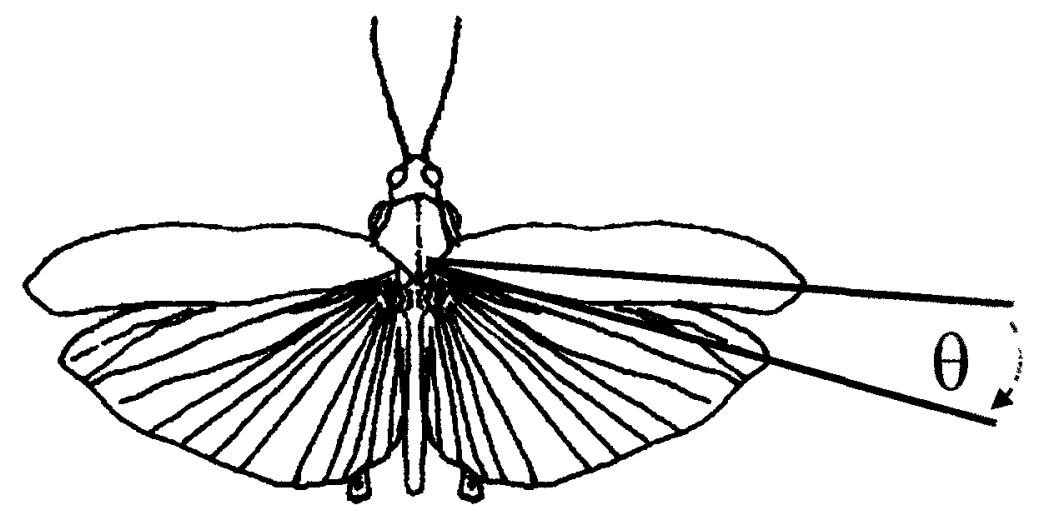

Figure 1.6. Drawing of a locust (dorsal view) illustrating separation angle $(\theta)$ of the forewing and hindwing. Separation angle is measured from the wing tips to the wing base for each wing. 
distance from the wing base to where the vortex re-attaches to the surface of the wing increases, which in turn delays stall (prevents the wing from stalling at low angles of attack), allowing for high lift generation (Ellington et. al., 1996). This delayed stall is a mechanism that allows for lift generation beyond the capacities of conventional wings in steady motion (Ellington, 1984b; Ellington, 1999). This is to say, airplanes have high lift coefficients $\left(\mathrm{C}_{\mathrm{L}}\right)$ at smaller angles of attack but are limited due to the fact that they stall at relatively small angles of attack compared to insects. It is speculated that wing camber (i.e. the flexion of the wing) is associated with the ability of producing greater angles of attack without stalling (Wooton et. al., 2000, Smith et. al., 2000). Schnackenburg et. al., (unpublished, 2007), showed that isolated locust wings in rotation at angles of attack up to $100^{\circ}$ are still able to produce lift due to the flexibility and camber of the wing; however, flying locusts don't typically exhibit angles that extreme.

\subsubsection{The Clap-Fling Mechanism}

The clap-and-fling (CF) was first described by Weis-Fogh (1973). It's a phenomena observed during take-off by moths, butterflies, locusts and large birds (i.e. pigeons). During take-off, the wings are elevated to an extreme dorsal position then rapidly 'flung' open. The sudden in-rush of air into the opening gap between left and right wings rotates into a vortex that produces lift force normal to the wing surface (Ellington, 1984a). This is therefore a special case of the LEV. Other animals (insects) vary this pattern into a 'peeling apart' of the wings and are thus able to direct the lift force in a particular direction. There is significant cost to lift production via this mechanism - as wings touch 
(and collide), damage can occur to the wings.

\subsubsection{Wing Rotation}

Wing rotation is a mechanism that produces lift at the stroke reversal, where the wings rapidly rotate to establish the correct angle of attack for the downstroke. Pronation (occurring at the start of a downstroke) or supination (occurring at the start of an upstroke) is very rapid and is thus sometimes referred to as the 'wing-flip' (Dickinson et. al., 1999, Goth, 2010). The timing of the wing-flip and angle of attack of the wing is crucial in determining the associated lift forces and has been shown in Drosophila that flipping the wing slightly prior to the stroke reversal is the most effective in producing additional lift. Note that the lift enhancement is brief and occurs at the moment of the stroke reversal. This is why we speculate it is important for generating steering torques. Goth (2010), has shown that it is not a phenomena limited to fruit flies, locusts also use this mechanism during steering.

\subsubsection{Wake Capture}

Wake capture (Dickinson et. al., 1999) is the third-high-lift generating mechanism and occurs only in insects with very high wing beat frequencies that flap their wings in mostly horizontal stroke planes. This mechanism therefore is unlikely to be occurring in locusts. During wake capture, the shed vorticity caused by one wing stroke lingers in space behind the insect and is actually 're-captured' by the rapidly beating wings. The 
reaction forces generated by the wing passing through this lingering vorticity can be used to enhance lift production during the current stroke. This is analogous to 'treading water' in a swimming pool.

\subsubsection{Wing-Wing Interactions}

Wing-wing interactions, specifically aerodynamic interactions between ipsilateral wing couples, the topic of this thesis, are poorly understood to date. To this author's knowledge, there are no publications to date on such wing-wing interactions in flying locusts. It's a new area of focus because it couldn't really be studied until sophisticated PIV techniques were widely available. The literature three principle lift generating mechanisms (as previously mentioned), but how are those mechanisms affect by wingwing interactions, and are the interactions between the forewings and hindwings beneficial or detrimental in the production of lift forces? Dawson, Murphy and Ellington were the first to hypothesize that that angular difference between the wing pair might affect the development of a separation bubble (LEV) on the hindwing (Murphy, 2005). This hypothesis was followed by some preliminary data collection by Schackenburg et. al. (unpublished, 2007), which showed that LEV's are being formed on both the fore- and hind wings when in isolation and at certain angular differences when paired together. It also supported the idea that LEV formations were dependent upon the angular difference as well as the angle of attack of the wings. They observed that with large angular differences, as the angles of attack were increased, the amplitude of the LEV's also increased They also observed that LEV's from the forewing did not interact with the 
LEV's of the hindwing until the angular difference was sufficiently low to allow them to interfere $\left(>\sim 20^{\circ}\right)$.

Wang and Sun (2005) looked at the impact of phase relationship on wing-wing interactions in dragonflies using computational analyses. They found that the interactions were detrimental to vertical force and hence, resultant force production in hovering dragonflies and when advance ratio's were very small. They found that lift production increases as advance ratio increases.

A re-occurring theme has developed among studies focusing on wing-wing interactions, and that is that the interactions are dependent on the phase relationship of the wings and are impacted by wing stroke kinematics (Wang \& Russell, 2007, Lehmann \& Pick, 2007, Lehmann et. al., 2005, Deng and Hu, 2008).

\subsection{Dragonfly Flight}

Dragonflies have been used as a model species to study the aerodynamics of fourwinged insects because they have a long history of wing kinematics, they show various flight capabilities such as hovering, forward flight and incredible maneuverability. They can modulate the phase lag between the fore and hind wings allowing for the variations in flight. Hovering is easier to study mathematically because the velocity is equal to zero; therefore, all the equations involved in calculating parameters involved in flight become simplified, once again making dragonflies an ideal specimen to study aerodynamics.

Wang and Russell (2007), showed that dragon-flies can alter the phase relationship (which essentially translates to angular difference) between the fore and hind wings 
which results in different flight behaviours. A phase $(\varnothing)$ of $0^{\circ}$ occurs when both the foreand hindwings are beating together simultaneously resulting in the maximum force production and power output needed for takeoffs for example. When the fore- and hindwings beat in phase, a single LEV is formed and is much larger than would be individually on each wing due to the increase in surface area of the combined wings (Thomas et. al, 2004). A phase of $\varnothing 180^{\circ}$ results in the wings beating opposite one another so the net force will balance the weight of the animal resulting in a hovering state.

Dragonflies will fly with a relatively horizontal stroke plane which allows them to hover due to the fact that they can produce lift on both the upstroke and the downstroke. This means that wing-wing interactions may be occurring constantly in a dragonfly. In contrast, locusts fly with a steeply inclined, almost vertical stroke plane (Figure 1.1) and only produce lift throughout the downstroke. They rotate their wings to a steep angle of attack during the upstroke to minimize drag forces. These differences need to be taken into consideration when studying four-winged insects alike as they may have varying aerodynamic consequences.

Somps and Luttges (1985) were the first to consider that large force production in dragonflies during flight may be due to the effect of forewing-hindwing interactions.

\subsection{Methods for Studying Insect Flight}

Many of the studies conducted on the aerodynamics of insect flight employ flow 
visualization techniques to observe the fluid movement around the insect during flight. The flow fields in water are easier to visualize that flow fields in air, therefore; mechanical models are developed to reproduce the wing kinematics of an insect that can be operated under water. Additionally, flight is studied in liquids so that models can be scaled. As long as the Reynolds number $(\mathrm{Re})$ of the model in water matches the Re of the insect in air, then the fluid structures will be the same. To produce an accurate mechanical model for flow visualization techniques, kinematic data collected and morphological measurements from real locusts are applied. Reynold's number, grossly speaking, is the ratio of inertial forces to viscous forces acting on a body. A low Re indicates a dominance of viscous effects of the fluid flow (air or water) on an animal, whereas a high $R e$ is indicative of a dominance of inertial effects of the animal over fluid structure. To be sure a model is operating as the real locust, it is critical that the Re of the model equal the Re of the real locust. Re is calculated for a locust by methods based on the following standard equation for Reynolds Number (Re):

$$
R e=\frac{\rho v c}{\mu}
$$

Where $\rho$ is the density of air, $v$ is the velocity of the object moving through stationary air (i.e. a flying locust), or the velocity of air moving across a stationary object (i.e. a wing tunnel), $c$ is the cord length of the wing and $\mu$ is the dynamic viscosity of air (Vogel, 2003). The Reynolds number of the body of a migratory locust is Re 12,300 with the forewing operating at an $\operatorname{Re}$ of $\sim 2800$, and the hindwing at an $\operatorname{Re}$ of $\sim 4300$ (Dudley, 2000).

Dye-flow visualization and V3V are two techniques used that allow for the visualization of flow fields. The majority of these techniques are conducted in either a 
spinner tank or a tow tank. Dye-flow visualization techniques involve placing an array of dye sources at the leading edge of a model wing. When the dye is released, the flow field can be visualized by the streams of colour in the fluid. V3V is a technique that involves seeding the fluid in the tank with reflective, neutrally buoyant particles and illuminating a volume of particles in the fluid using a laser, which is focused into a cone of light centered in front of a camera. Both techniques allow the gross structure to be observed; however, the V3V technique allows researchers to obtain quantitative data in comparison to dye-flow, which is a better technique to obtain qualitative observations. When using the dye-flow technique, dye streams linger, so qualitative observations can be made of changes occurring as the model rotates or is towed and some quantitative observations such as the location of vortex formation and the sense of the vortices can be determined. The dye-flow technique is relatively easy, inexpensive and accessible in comparison to the V3V system (costly, requires technical expertise). The V3V system also has its advantages, it is a 3-dimensional system, therefore; quantitative analysis of vectors can be calculated allowing for predictions of force output using the following equation:

$$
F \propto \rho s \nu \Gamma
$$

Where $\rho$ is the density of air, $s$ is the span of the airfoil, $v$ is the velocity of the object and $\Gamma$ is the circulation defined as the circumference of the isovelocity contour in the flow field multiplied by the velocity along the closed loop of the circumference (Alexander, 2003).

\subsection{Flapping Models versus Rotating Models}


In flapping flight, many of the kinematics constantly change during forward translation (mainly the downstroke and upstroke). For example, the angle of attack is maximized during the downstroke to produce lift, and minimized in the upstroke to reduce drag (Vogel, 2003). In four-winged insects, the phase relationship (i.e. angular difference) is constantly changing as well. As a result, many of the mechanisms involved with lift production, such as the clap-fling mechanism and wake capture, are particular to flapping flight and cannot be observed in rotating wing models. In fixed, rotating wing models, there is no translation and no stroke reversals, so the wings are then mimicking a constant downstroke phase. Although this is not representative of forward flapping translation, it does depict an insect that is hovering and an insect that exhibits a vertical stroke plane (such as a locust) that is ascending in a steeply inclined direction. It benefits in the observation of structures such as the leading edge vortex due to the fact that there is no shedding of the vorticies as a result of stroke reversal, although shedding is still occurring at the tip vortex.

In flapping flight, two velocities are taken in to account, wingtip velocity (UT) and forward velocity of the wing ( $\left.U_{R}\right)$. The base of the wing would ideally have a UT of zero $\mathrm{m} / \mathrm{s}$ as it remains in a fixed position on an apex; therefore, only forward velocity would affect the base of the wing. In contrast, the wingtip would have a velocity (UT) as a result of the flapping motion (upstroke and downstroke) and the forward velocity of the wing (UR) (Figure 1.7).

Rotating wings remain in a fixed position as they rotate along a central shaft. This simulates a constant downstroke where the neither the wing tip nor the wing base experience a tip velocity (UT), and the forward velocity ( $\left.U_{R}\right)$ is increasing along the 
A

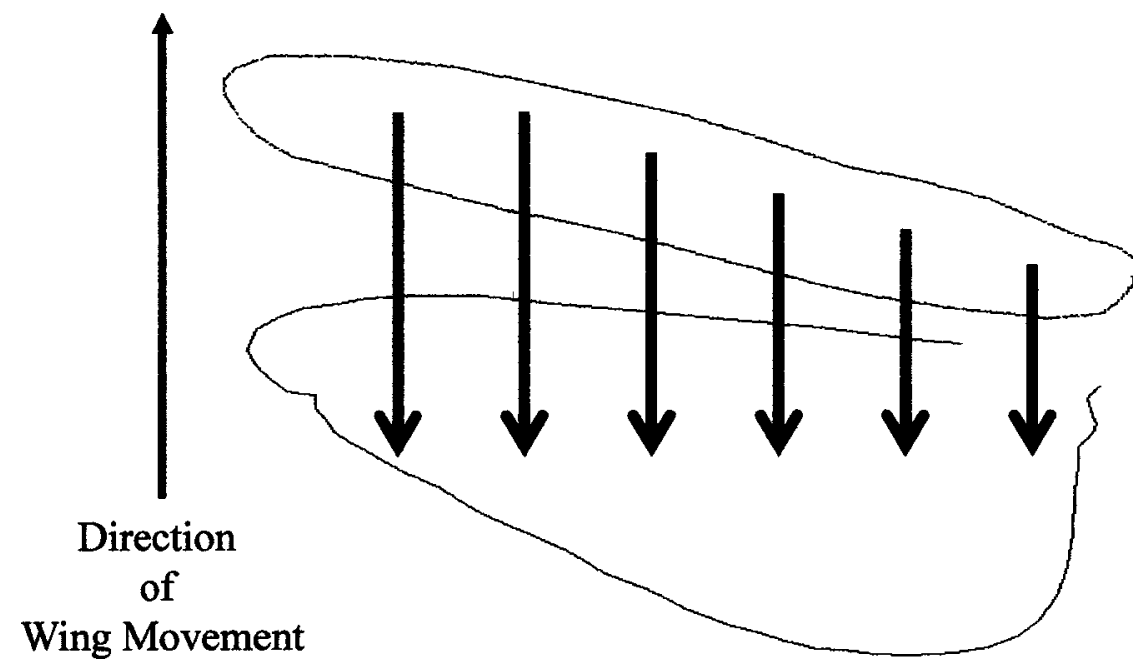

B

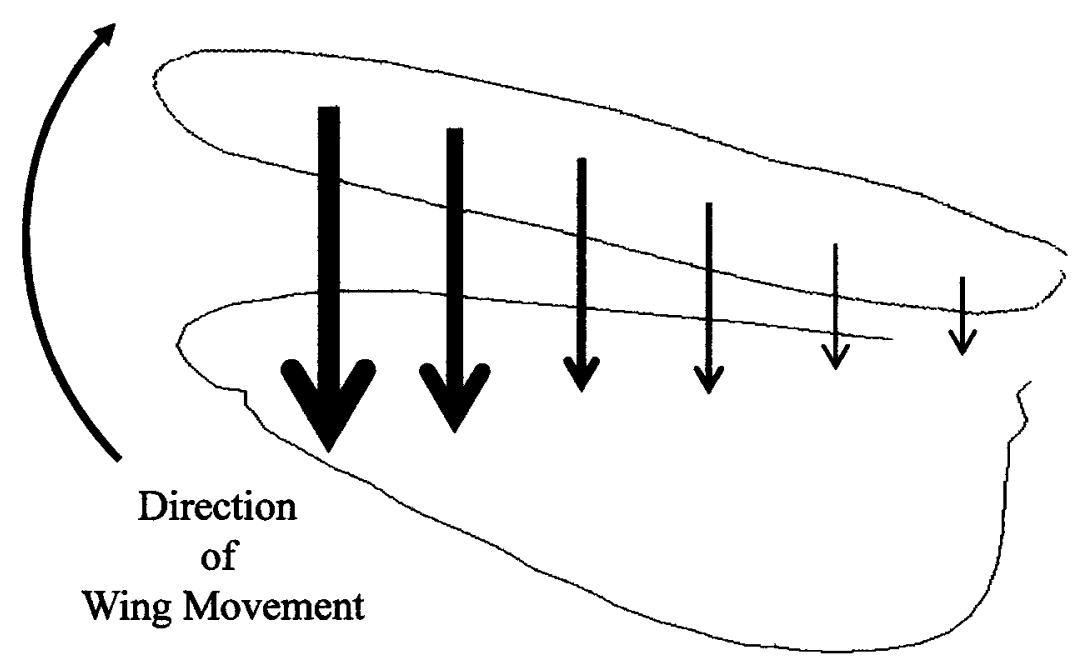

Figure 1.7. Schematic representation of chord wise flow of a flapping wing in forward translation (A) and one in rotation (B). The velocity gradient from base to tip in rotating wing models is larger than in forward translating wings. 
length of the wing from base to tip, resulting in a velocity gradient along the wing (Figure $1.7)$.

\subsection{Locusta migratoria}

\subsubsection{Life History}

The African migratory locust (Locusta migratoria L.) is found natively in equatorial regions of Africa, Asia, India, and Australia (Chapman, 1982). It is a member of the order Orthoptera, family, Acrididae, subfamily Oedipodinae. There are two different phases for locusts, gregarious phase and solitary phase. Their fate is decided upon their aggregation behavior and influences such as physical contact and visual and olfactory cues from other locusts will trigger the phase formation (Lester et. al., 2005). Gregarious phase locusts consist of large swarms of the insect, where as solitary phase locusts can live independently and be widely dispersed (Chapman 1982). Locusts have four wings, two forewings and two hindwings. The centre of body mass of a locust is located posterior to the thoraco-abdominal junction (Dudley, 2000).

\subsubsection{Using Locusts to Study Flight}

Locusts are very robust insects and in combination with their relative size and ease of maintaining a colony (Barass, 1974). They make an ideal specimen to use for insect flight experiments. Gregarious phase locusts fly readily which aids in collecting 
data obtaining to wing kinematics and mechanics of flight. The solitary phase locusts fly at night and do not naturally have the drive to fly for long periods of time; therefore, do not exhibit the same flight capabilities and behaviours as gregarious locusts who fly during the day.

Locusts have been a relatively common model used for studying insect flight due to their relatively ease of handling, colony maintenance and the fact that they have a comparatively simple nervous system. Their simple nervous system make it easier to identify muscles and their associated neurons which make them an ideal insect to study neuro-musculature systems; however, the aerodynamics involved in locust flight are still not well understood. This is likely due to challenges of understanding wing-wing interactions. In two-winged insects, such as flies, bees, etc., kinematics are easier to obtain as there are no wing-wing interactions involved.

Locusts make for an interesting model insect to study because they respond to 'bat-like' ultrasound acoustic stimuli by performing an evasive maneuvre called acoustic startle response (ASR) (Robert, 1989), which allows researchers to readily study the aerodynamics involved in steering. Locusts also respond to visual stimuli (Robertson \& Reye, 1992, Robertson \& Johnson, 1993) and thermal stimuli (Robertson et. al., 1996, Dawson et. al., 1997, Robertson \& Shoemaker 1998); however, an acoustic stimulus is easily controlled by maintaining a constant intensity, frequency and envelope, as well it has biological relevance making it an ideal stimulus to employ when studying locusts' ASR. 


\subsection{Tethering}

Two types of tethers are typically employed when studying locusts, rigid tethers and loose tethers; more commonly used throughout the literature are rigid tethers. Ideally, locusts should be studied under free flight conditions where they are not bound or restricted in their flight maneuverability (Kutsch \& Fuchs, 2000); however, at the present time, this ideal condition is near to impossible to record and analyze for accurate kinematic data because locusts will not remain in the same position during flight and therefore the world coordinates needed for analysis would need to be calculated and adjusted for each captured frame during flight. A calibration apparatus to allow for such a technique does not currently exist. At best, qualitative observations can be made from an experimenter at a distance during free flight of a locust.

A rigid tether is easier to work with and makes it easier for the animal to stabilize itself in the laminar flow of the wind tunnel during flight, allowing it to enter a tucked flight position more readily; however, the animal is restricted on its mobility to react to an acoustic stimulus. A rigid tether does not allow for any pitch or yaw movement of the locust and it is therefore fixed in a forward flight position. Recordings of abdomen deflection and dorsiflexion as well as wing stroke amplitude, wing beat frequency and forewing asymmetry allow researchers to evaluate the intended steering direction and escape maneuvers of a locust that is rigidly tethered.

A loose tether allows for the animal to pitch and yaw during flight and some loose tethers even allow for rotation to permit the locust to turn in a chosen direction during an evasive maneuver. The loose tether allows the animal more variability in its flight 
patterns, especially during the acoustic startle response; however, it is more difficult for the animal to stabilize itself in the laminar flow and assume a tucked flight posture and remain steady in the flight posture therefore at times rendering the animal fatigued before the trial even beings. Typically, straight forward flight is assumed prior to any acoustic stimulus, and using a loose tether can lead to animals flying in a bias direction prior to a stimulus being applied making it difficult to evaluate the steering direction and maneuvers during an acoustic startle response. Using any type or tether, rigid or loose, invariably leads to biases in the flight pattern. This is an unavoidable consequence of the necessity to restrain an animal for observation.

Tethering effects have been shown to affect certain flight kinematics during an acoustic startle response (Fauquier, 2008). Kinematics such as wing stroke amplitude, forewing asymmetry and wing beat frequency are among some of the characteristics that show statistically significant differences among several different rigid and loose tethering methods (Fauquier, 2008).

\subsection{Thesis Objective}

The overall objective of my thesis is to document and characterize the aerodynamic interactions between the locust forewing-hindwing pair during flight by specifically focusing on leading-edge vortex (LEV) formation on both wings. The LEV is primarily responsible for the lift production in flying locusts (Ellington, 1996), and since the hindwing is the major contributor to lift in locusts, three predictions can be inferred:

1) The LEV on the hindwing will be completely destroyed due to the interactions of flow 
from the forewing, 2) the LEV on the hindwing will be augmented due to the flow interactions from the forewing, and 3) the LEV on the hindwing will be impeded or delayed in development along the length of the hindwing as a result of the wing-wing interactions. I hypothesize that the third prediction will likely be the case.

I believe that studying the aerodynamics of locusts will provide new insights into insect flight, and more specifically, into wing-wing interactions. Wing-wing interactions have been shown to effect lift production in flying insects, for example aiding in hovering and maneuverability in dragonfly flight; however, it is unknown how these interactions contribute to locust flight.

In this study, I will use high-speed cinematography, dye flow visualization and volumetric 3-component digital particle image velocimetry (V3V-dPIV) to thoroughly describe wing-wing interactions between the fore- and hindwings of the locust (Locusta migratoria Linneaus). This research is important because it will help in our understanding of the aerodynamics of all insects and why insects are such a successful group of animals. My findings are also important to other fields like engineering for future applications such as the development of bio-inspired micro-air vehicles. 


\section{Chapter 2: Wing-Wing Interactions in Rotating Locust Wings}

\subsection{Introduction}

Despite locusts being a model for insect flight studies since the 1950's, a comprehensive understanding of locust flight is yet to emerge. This is surprising given the advances seen for fruitflies (Dickinson et. al., 1999), hawkmoths (Ellington, 1997; van den Berg \& Ellington, 1997), and certain other insects (Ellington, 1984). The problem lies in the fact that locusts represent a more challenging model in two key factors: first, locusts are functionally four winged, and second, the interactions between forewings and hindwings in locusts, given their steeply inclined wing stroke plane during forward flight, has until now been largely intractable.

The first steps in solving these problems are to visualize the aerodynamic interactions between locust wing couples. This is the primary goal of this chapter. I will use model wing couples rotating in water to simulate the locust wing couple during mid downstroke translation. Both dye and digital PIV will be used to visualize and quantify the fluid interactions between the wing couples. A wing couple 'frozen' in time at this moment is definable via four variables: forewing angle of attack, hindwing angle of attack, forewing downstroke angle relative to hindwing downstroke angle (angular difference), and the separation of the forewing from the hindwing between respective stroke planes (separation angle). In these experiments, these variables will be manipulated; however, to ensure that I am using biological relevant values, 3-D 
kinematic reconstructions of the complete wing stroke of semi-rigidly tethered flying locusts in a wind tunnel will be completed.

\subsubsection{Kinematics}

Unfortunately, despite the vast literature on insect flight, and indeed locust flight spanning from the 1950's to present, studies are sadly lacking in controlled observations of key kinematic variables (Dawson, 2001) necessary for a comprehensive model (mathematical or conceptual) of forward flight to be developed. For example, the relationship between stroke plane angle and wing stroke amplitude is not known for locusts in ascending and forward flight as are how these variables change as a function of forward flight velocity. A detailed set of observations relating the angle of attack of the forewings and hindwings as a function of stroke angle is also lacking. Much of these limitations are due to the absence of a controlled environment for free, unrestrained flight, which would allow for more detailed and relevant observations. Wang and Sun (2005) were the first to provide kinematic analysis of dragonflies during flight in a 3dimensional system; however, detailed quantitative kinematic analysis of locusts in forward flight or steering has not yet been done in 3-dimensional space. Analyses of locust kinematics in a 2-dimensional system may be misconstrued as a result of perspective issues (Figure 2.1). It is therefore important to obtain kinematic values in a 3dimensional system to eliminate the possible errors that could occur in a 2-dimensional system.

The purpose of the kinematic portion of my thesis is twofold - first to collect 


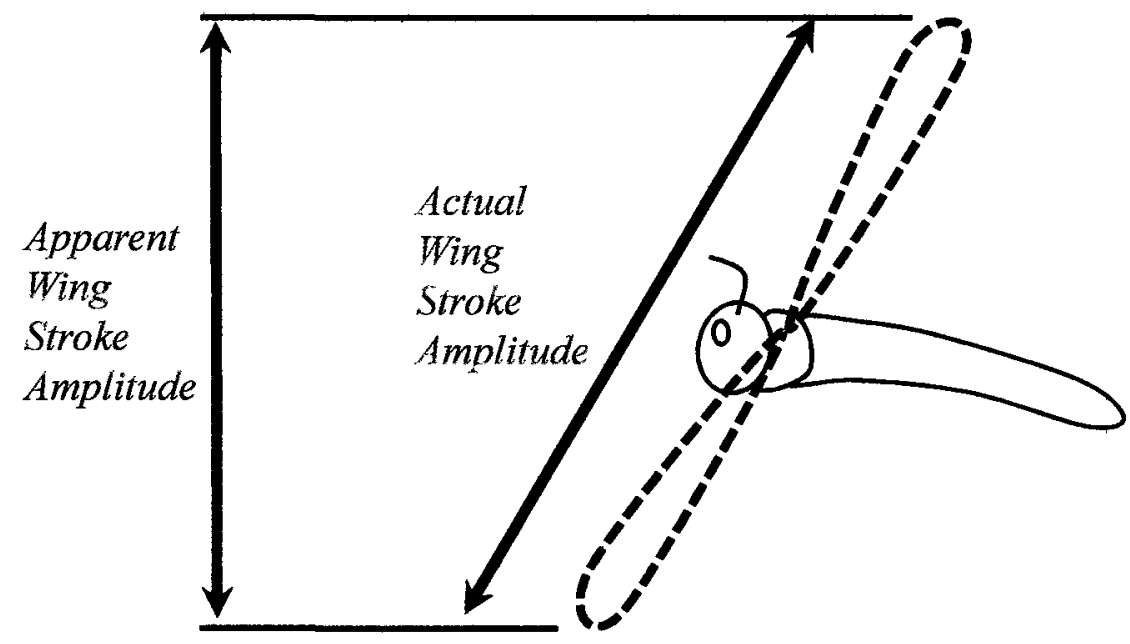

Figure 2.1. Perspective issues with observing wing stroke amplitude from a single camera positioned behind the locust. Using two cameras and 3-D kinematic reconstruction techniques eliminates perspective issues. Note, only the forewings are shown for clarity. 
accurate kinematics of real locusts flying (tethered) in forward flight and while steering (in response to an acoustic stimulus) in 3-dimensions, and second, to obtain kinematic data for the accurate construction of an articulated model for further flow visualization studies.

Specifically for my thesis objective of describing wing-wing interactions, I will measure the angular difference (fore- hindwing gap angle), the separation angle and the forewing asymmetry of locusts in straight flight and while steering in response to an acoustic stimulus. These measurements will need to be made at $1 / 3$ the downstroke where it is said that locusts produce the greatest amount of lift (Dawson et. al., 1997). Wing stroke amplitudes and wing beat frequency will also be measured at the peak of the upstroke to the peak of the downstroke during forward flight and while steering.

\subsubsection{Dye Flow Visualization}

Dye flow visualization allows for the observation of the gross flow structure surrounding a moving object by entraining streaks of dye in areas of low pressure. Placement of dye crystals along the surface of the object is crucial for visualizing flow structures. For example, in the case of insect wings, in order to view LEV structures forming along the leading edge of the wing, dye should be placed at the base of the wing where the dye can be entrained along the low-pressure zone of the LEV formation. There are limitations to this technique, one of them being that it is difficult to extract quantitative data from dye-flow visualization. The majority of observations are strictly qualitative. Another limitation is that the visualization of flow structures are dependent 
on the location of the dye placement; therefore, improper or lack of placement could potentially lead to structures not being viewed to their entirety or not being viewed at all.

Dye-flow is ideal for obtaining preliminary observations of flow structures to be applied to systems that are more easily capable of achieving quantitative results such as the volumetric 3-component velocimetry (V3V) system. It allows you to specify an area of interest, in this case, LEV formation on the fore- and hindwing pair, to be measured in further experimental studies.

Lu et. al. (2006), were the first to clarify the existence of two LEV structures forming along the leading edge of a wing in motion. They termed them the minor LEV and the primary LEV. As with this study, Lu et. al. used dye-flow visualization to confirm the presence and observe the LEV structures, and then they used digital particle image velocimetry (dPIV) to reveal substructures and quantitative measurements of the LEV's. They determined, for the first time that the minor LEV runs parallel to the leading edge of the wing at an angle of $0^{\circ}$ and although is said to always be present, may not always be visible due to the weakness in vorticty and inability to entrain the dye as a result of lower angles of attack. The primary LEV runs at an angle relative to the angle of attack of the wing and forms independently from the minor LEV at the base of the wing and that both LEV structures are insensitive to aspect ratios $(A R)$.

Lu et. al confirmed the presence of the dual LEV structures on flapping wings in isolation. The purpose of the dye-flow visualization experiments is to evaluate how those LEV structures are influenced with the presence of the paired wing like that of a fourwinged locust. In other words, how the presence of the forewing affects the development of the structures on the hindwings and 'vice versa' while manipulating variables such as 
separation angle, angular difference and wing angle of attack. The results will give a better understanding of the overall flow structures being affected by wing-wing interactions and this knowledge can be applied to the V3V section of this thesis for further, in depth analysis of specific areas of interest.

\subsubsection{Volumetric 3-Component Velocimetry}

Volumetric 3-component velocimetry (V3V) is a system capable of quantifying flow around a moving object, in this case, a locust forewing and hindwing rotating along a stationary shaft. This imaging technology is relatively new to the science community and uses similar concepts as a more familiar technique called digital particle image velocimetry (dPIV). The key difference lies in the area of the object captured where if one could imagine a loaf of bread, using the dPIV technique, only one slice of that loaf could be captured at a time and eventually all the slices could be compiled together to complete the loaf, but the V3V system would be able to capture the entire loaf in one instance. In other words, $\mathrm{dPIV}$ is a 2-dimensional particle tracking technique and V3V is a 3-dimensional particle tracking technique where the V3V system measures an instantaneous velocity field within a volumetric region.

The V3V system works by tracking particles illuminated by a laser that are disturbed by the flow structures of the rotating wings. Using algorithms in a software program specifically designed for this technology, the size, magnitude and direction of each flow structure involved in the wing-wing interactions can be measured. This advanced technology should complement the qualitative observations seen using the dye flow visualization technique and provide quantifiable data to the observations. 
The purpose of this section of the study is to provide velocity magnitude measurements of the LEV structures on the hindwing, which may or may not have been affected due to presence of the forewing and the manipulation of the multiple variables discussed in the dye-flow chapter.

\subsubsection{Rotating Wing Models}

A spinner tank involves a mechanical model rotating in a fluid (Figure 2.2). The rotation of the model produces a velocity gradient along the wing, which includes: 1) a chord-wise velocity because the cord of the wing cuts through the water as it rotates, and 2) a spanwise velocity gradient which results from the wing base having a different velocity than the wingtip. It is easier to study wing kinematics using a rotating model because a rotating wing model is effectively a wing in translation only - there are no stroke reversals. There are differences - the model is at a constant velocity (a real wing accelerates and decelerates during translation), the model is at a constant angle of attack (a real wing changes angle of attack during translation), and, as noted above, the flows are different over the wing. Ideally rotating wing models would be used to study translation of a wing on a hovering insect, and although locusts lack the ability to hover; therefore, this technique would not accurately depict a locust in straight forward flight, locusts do exhibit these characteristics in steering and steep ascent (such as take-off).

Flapping motions are not taken into consideration with a fixed, rotating wing model; therefore the results are not necessarily representative of forward flight. With a rotating model, there are no span ratios (the ratio of the wing span on the upstroke to that 

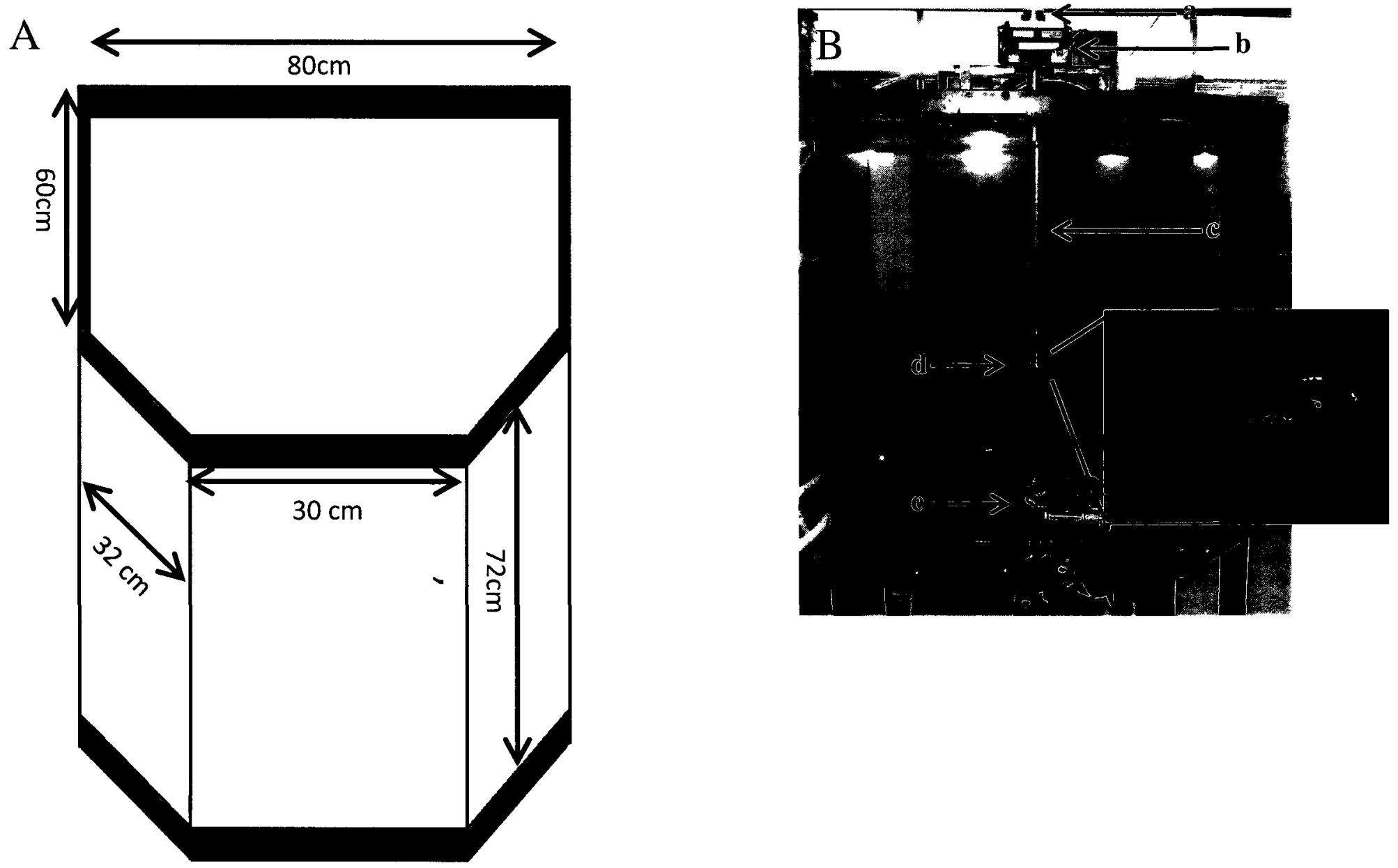

Figure 2.2. (A) Diagram illustrating the dimensions of the spinner tank (capacity c. 500L). (B) Set-up of the spinner tank showing the DC motor (a), digital motor sensor display (b), shaft of the spinner (c), mounted wing pairs (expanded to show wing pair models) (d), and finally the tripod placement for the high-definition camera (e). 
on the downstroke), and no shedding of the vortices at the stroke reversal (Usherwood \& Lehmann, 2008). The vorticies are still shed at the tip vortex, but the shedding is occurring constantly as the rotation speed is constant. Rotating wing models therefore represent a quasi-steady state of aerodynamics. 


\subsection{Materials and Methods}

\subsubsection{Kinematics}

\subsubsection{Animals}

African migratory locusts (Locusta migratoria L.) used throughout this experiment were obtained from a colony maintained in the Biology Department at Carleton University. Locusts are reared in wood cages measuring $43 \times 41 \times 46 \mathrm{~cm}$ with lights (16h:8h light:dark cycle) that maintain a temperature between $28^{\circ} \mathrm{C}$ and $35^{\circ} \mathrm{C}$; the preferred temperature for the locusts in their natural environment (Barrass, 1974). They are fed a diet of wheat grass, carrots and a mixture of rolled oats, yeast and skim milk powder ad libitum. Locusts are reared under crowded conditions (more than 30 individuals per cage), which maintain the gregarious phase due to frequent physical contact and visual and olfactory cues from numerous other locusts in the environment (Lester et. al., 2005). I used gregarious phase locusts throughout this experiment as they are more likely to fly for longer periods of time as per their natural behaviour of flying in large swarms (Barass, 1974).

Only adult locusts were chosen that were approximately at least 2 weeks past imaginal ecdysis. Further, only locusts with both forewings and hindwings intact with no damage, as well as possessing all legs and both antennae were selected for inclusion in the study. No preference was given to the sex when selecting animals. Although there are 
some morphometric differences between males and females and sometimes differences in flight duration capabilities, due to the fact that the locusts will be flown for short durations in the wind tunnel, these differences are irrelevant for the purposes of this study.

After selection from the colony, locusts were kept in clear plastic containers measuring $12 \times 12 \times 10 \mathrm{~cm}$ with an aluminum screen top. To prevent any hindrance of the flight muscles from being too cold from room temperatures and potentially affecting their flight capability, locusts were pre-warmed for flight tests using a halogen light, which kept temperatures between $27^{\circ} \mathrm{C}$ and $33^{\circ} \mathrm{C}$ within the container.

Locusts were used for only one flight trial and were stimulated with brief physical contact on the head after each acoustic stimulus to prevent habituation of the stimulus. After each trial, the sex, weight, pronotum length, head capsule length, femur length and forewing length were recorded (Table 2.1).

\subsubsection{Tethering of Specimens}

Two types of tethering methods were employed for kinematic data collection: a rigid sternal tether and a semi-rigid ventral tether. Both tethers are fixed at the centre of mass of the locust. The rigid sternal tether consists of a sculpted aluminum rod with a flat tip ventrally affixed to the locust at its abdomen-thorax junction using a drugstore brand of hair removal wax (Parissa ${ }^{\circledR}$ strip-free hot wax) This is suitable as it has a low melting 
Table 2.1. Summary of locust morphometrics, acoustic stimulus characteristics and wind tunnel characteristics.

\begin{tabular}{|c|c|c|c|c|c|c|c|c|c|c|c|}
\hline Locust & Sex & $\begin{array}{l}\text { Weight } \\
\text { (g) }\end{array}$ & $\begin{array}{l}\text { Pronotum } \\
\text { Length } \\
\text { (mm) }\end{array}$ & $\begin{array}{l}\text { Head } \\
\text { Capsule } \\
\text { Width } \\
(\mathrm{mm})\end{array}$ & $\begin{array}{l}\text { Forewing } \\
\text { Length } \\
\text { (mm) }\end{array}$ & $\begin{array}{c}\text { Femur } \\
\text { Length } \\
(\mathrm{mm})\end{array}$ & $\begin{array}{l}\text { Tethering } \\
\text { Method }\end{array}$ & $\begin{array}{l}\text { Loudness } \\
\text { (dB-SPL) }\end{array}$ & $\begin{array}{l}\text { Wind } \\
\text { Speed } \\
(\mathrm{m} / \mathrm{s})\end{array}$ & $\begin{array}{l}\text { Stimulus } \\
\text { Frequency } \\
(\mathrm{kHz})\end{array}$ & $\begin{array}{l}\text { Recording } \\
\text { Rate (fps) }\end{array}$ \\
\hline $09 / 24-1$ & $\bar{F}$ & 1.13 & 7.62 & 6.05 & 40.09 & 19.92 & Ventral & 82.31 & 3.9 & 30 & 1000 \\
\hline $09 / 24-2$ & M & 0.72 & 6.72 & 5.99 & 38.91 & 18.62 & Ventral & 82.31 & 3.9 & 30 & 1000 \\
\hline $12 / 11-1$ & M & 1.05 & 6.22 & 5.08 & 33.43 & 17.51 & Ventral & 83.29 & 4.0 & 30 & 1000 \\
\hline $12 / 11-2$ & $\mathrm{~F}$ & 1.40 & 7.23 & 6.16 & 41.42 & 19.13 & Ventral & 84.19 & 4.1 & 30 & 1000 \\
\hline $01 / 11-1$ & F & 0.90 & 6.94 & 6.46 & 36.92 & 18.66 & Sternal & 80.34 & 3.9 & 30 & 1000 \\
\hline $01 / 11-2$ & $\mathrm{~F}$ & 1.32 & 7.16 & 6.34 & 39.41 & 18.63 & Sternal & 80.34 & 4.2 & 30 & 1000 \\
\hline $01 / 11-3$ & M & 1.25 & 7.50 & 6.26 & 40.79 & 20.61 & Sternal & 80.34 & 4.2 & 30 & 1000 \\
\hline $01 / 11-4$ & M & 0.96 & 6.36 & 5.62 & 36.62 & 17.92 & Sternal & 80.34 & 4.3 & 30 & 1000 \\
\hline $01 / 23-1$ & M & 0.61 & 6.52 & 4.80 & 38.69 & 18.50 & Sternal & 84.71 & 4.0 & 30 & 500 \\
\hline $01 / 23-2$ & M & 0.60 & 6.31 & 4.65 & 37.72 & 17.86 & Sternal & 84.71 & 4.0 & 30 & 500 \\
\hline $10 / 11-1$ & M & 0.90 & 7.55 & 5.86 & 36.13 & 20.45 & Sternal & 84.71 & 4.0 & 30 & 500 \\
\hline $10 / 11-2$ & M & 1.00 & 7.57 & 6.35 & 42.29 & 20.21 & Sternal & 84.71 & 4.0 & 30 & 500 \\
\hline $10 / 11-3$ & M & 1.12 & 7.55 & 6.12 & 41.15 & 19.86 & Sternal & 84.71 & 4.1 & 30 & 500 \\
\hline $10 / 11-4$ & $\mathrm{M}$ & 0.58 & 6.48 & 5.66 & 36.22 & 18.45 & Sternal & 84.71 & 4.2 & 30 & 500 \\
\hline Average & & 0.97 & 6.98 & 5.81 & 38.56 & 19.02 & & 82.98 & 4.06 & & \\
\hline St. Dev. & & 0.268 & 0.535 & 0.59 & 2.51 & 1.01 & & 1.927 & 0.13 & & \\
\hline
\end{tabular}


temperature and a high adhesion to locust cuticle. Careful attention was paid to the area of wax application to prevent any from adhering to the wing hinges or neck region, which would prevent or impede their ability to fly. The locust was then positioned in the centre of the working section of the wind tunnel so that the locust's body was parallel to the wind stream (Figure 2.3a).

The semi-rigid ventral tether consists of a piece of galvanized steel wire that had been manually shaped into a hook. The tether was positioned on the side of the locust ventrally with a piece extending above the centre of the dorsal area of the locust. The tether was affixed to the locust by once again using the hair removal wax (Parissa ${ }^{\circledR}$ stripfree hot wax). The wire was inserted in to a plastic piece of tubing and attached to a metal rod (Figure 2.3b) supporting the locust in the wind tunnel.

To eliminate the possibility of visual stimuli interfering with the flight patterns and responses of the locust, opaque white paint (Wite Out ${ }^{\mathrm{TM}}$, BIC Corporation) was applied to the compound eyes and ocelli. Careful attention was paid not to get any paint on their antennae as they use their antennae to evaluate wind speeds and environmental conditions, which can influence their steering direction and flight path.

\subsubsection{Wind Tunnel and Acoustic Stimulus Set-Up}

A custom built low-speed (subsonic) laminar flow wind tunnel (fan blades are behind the working section of the wind tunnel) was used for kinematic data collection. The working section is $60 \mathrm{~cm}$ in length by $30 \mathrm{~cm}^{2}$ in cross section (Figure 2.4). The wind 

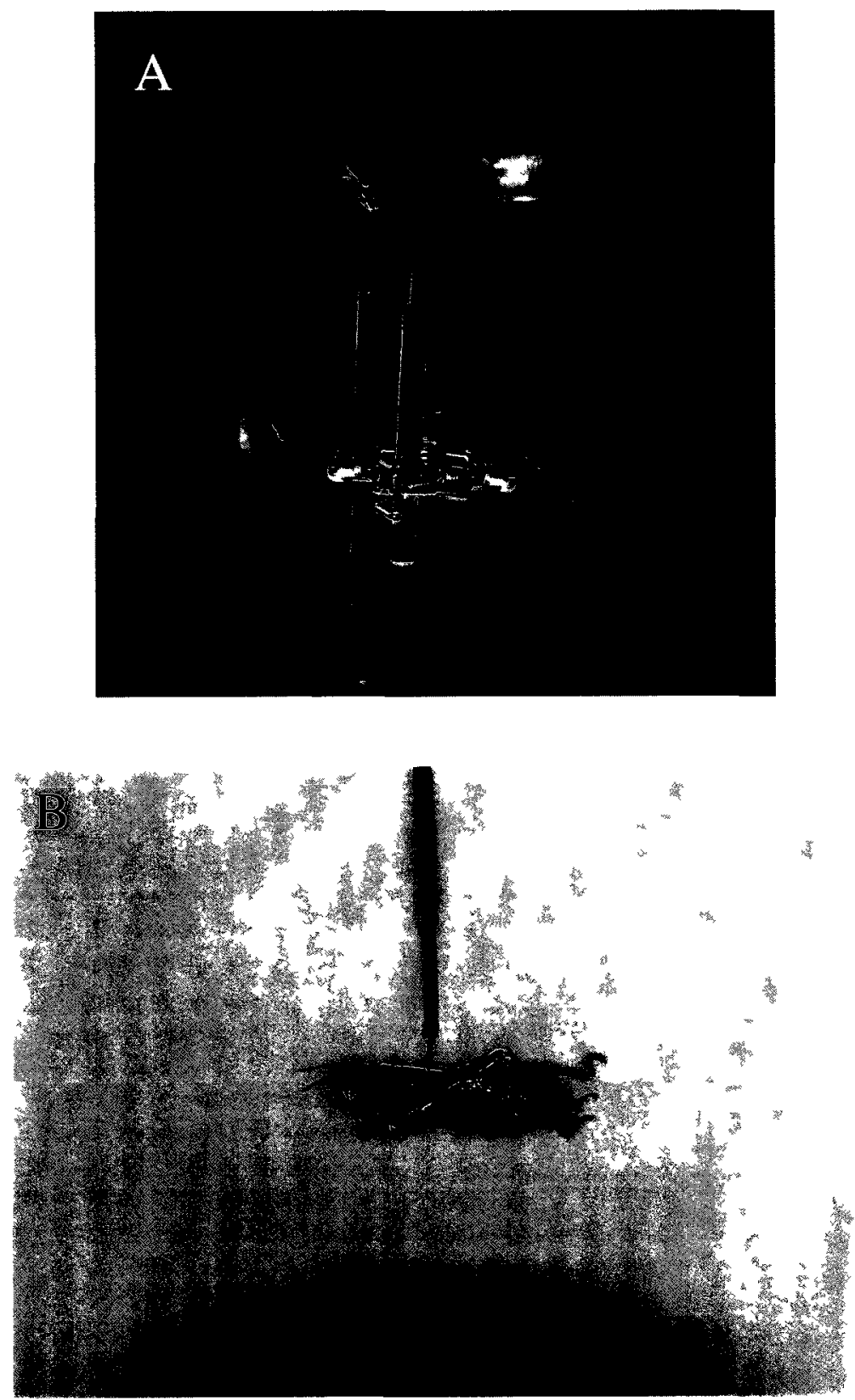

Figure 2.3. Photographs of the two types of tethering methods used in this study. The rigid sternal tether $(\mathrm{A})$. The semi-rigid ventral tether (B). 


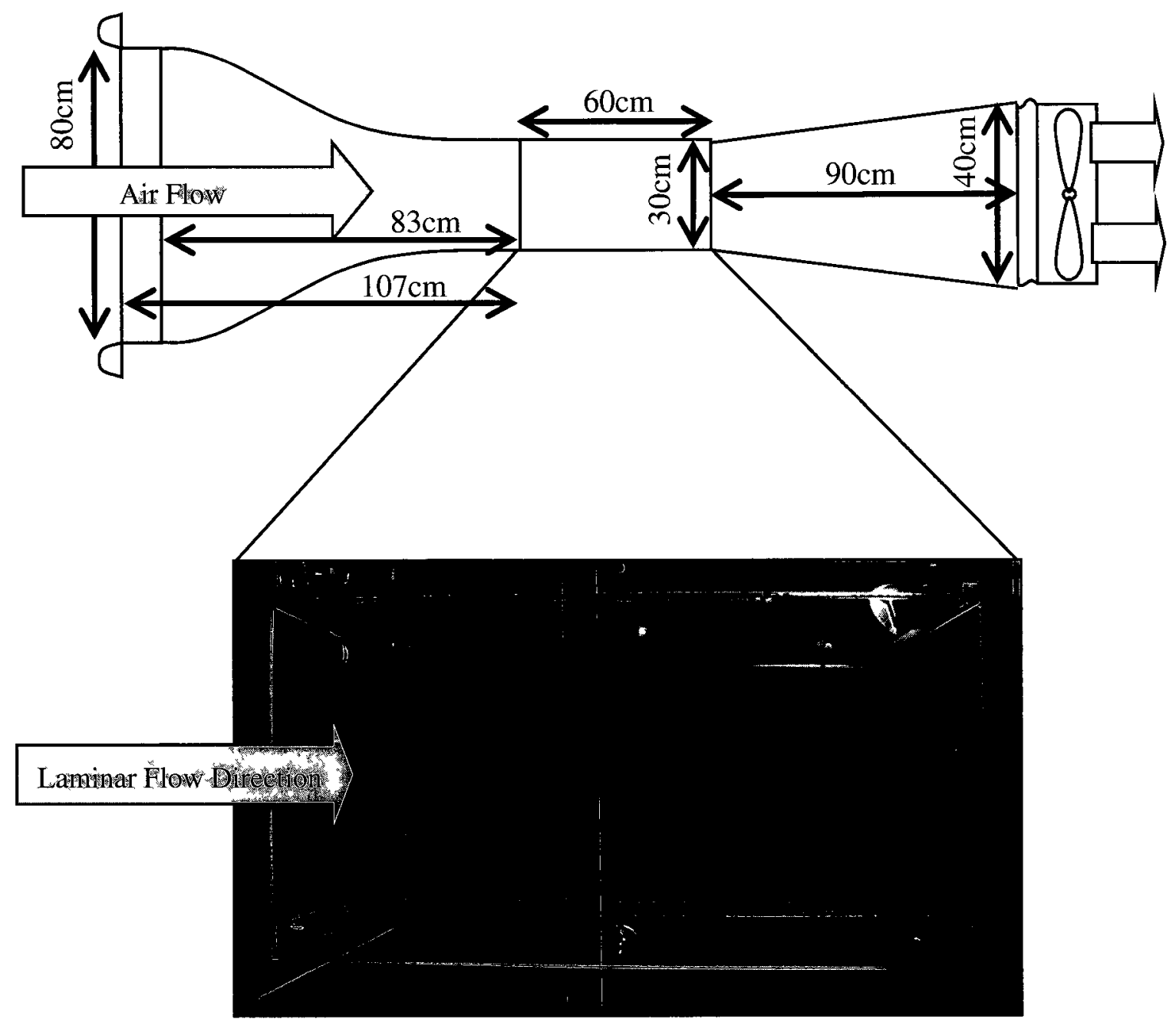

Figure 2.4. Schematic diagram of the custom built low-speed (subsonic) wind tunnel with a close up of the working section. Smoke in the working section illustrates the laminar flow of the wind tunnel. 
speed used throughout the trials for the locusts during flight was an average of $4.2 \mathrm{~m} / \mathrm{sec}$ (maximum $4.7 \mathrm{~m} / \mathrm{sec}$, minimum $3.7 \mathrm{~m} / \mathrm{sec}$ ). The wind tunnel speed and temperature were measured using hot-wire anemometer (VWR model 21800-024).

The acoustic stimulus was a train of $10 \mathrm{~ms}$ shaped pulses presented with a $30 \mathrm{~ms}$ period. The pulse trains were generated by synthesizing a sinewave of $30 \mathrm{kHz}$ carrier frequency (Tabor Wonder Wave, model 5061), then shaping the sine wave into individual $10 \mathrm{~ms}$ pulses each with a 1-ms rise/fall time (Coulbourn Selectable Envelope Shaped Rise/Fall Gate, model S84-04). Pulse trains were amplified by a custom built amplifier employing a Hi-Fi amplifier integrated circuit (NTE1380, NTE Electronics Inc.). The pulse trains were c.500ms which was triggered manually. The sound pulses were monitored by an oscilloscope (Tektronix, model TDS 2014) to confirm stimulus characteristics and to set the amplitude of the stimulus pulses. Stimuli were presented from a 2-inch cone tweeter (Motorola, model KSN1078A). The speaker was positioned $33 \mathrm{~cm}$ posterior from the locust and to the right. The sound intensity was set using a previously determined calibration of the tweeter speaker. Sound intensity calibration curves were constructed by measuring the intensity of pure tones of equivalent amplitude to the sound pulses used during experiments (Stapells et.al., 1982). Sound intensity was measured by placing a Brüel and Kjær 1/4" microphone (model 4135 without protective grid) at the same speaker distances used during experiments and measuring the sound intensity of the tones (in dB SPL re: $20 \mu \mathrm{Pa}$ RMS) on a Brüel and Kjær measuring amplifier (model 2209) corrected with a Brüel and Kjær pistonphone (model 4220). Intensities varied by trial and ranged between $80-85 \mathrm{~dB}$ (Table 2.1 ). 


\subsubsection{High-Speed Stereo Digital Cinematography}

Two camera (stereo) high-speed cinematography was used to capture the wing kinematics of all locusts during flight. Locusts were filmed at either 500 or 1000frames $\cdot \mathrm{s}^{-1}$ using paired Motion Scope high-speed digital cameras (model PCI1000S) each with a $6-\mathrm{mm}$ 1:1.2 TV lens shuttered at $1 / 1000$ or $1 / 2000^{\text {th }}$ of a second (Table 2.1 ). Two halogen lights (500 Watt), were placed outside of the wind tunnel, above the locust to facilitate image acquisition. Ambient temperature in the lab varies between $21^{\circ} \mathrm{C}$ to $26^{\circ} \mathrm{C}$ and the halogen lights increased the temperature of the working section of the wind tunnel an additional $10^{\circ} \mathrm{C}$. This is not a problem as the optimal temperature for Locusta migratoria (free) flight is between $28^{\circ} \mathrm{C}$ and $35^{\circ} \mathrm{C}$ (Barrass, 1974). High-speed camera footage was saved as uncompressed (RAW) AVI video format files.

For this study, the $\mathrm{x}$-axis is defined along the wingspan, the y-axis is the dorsalventral axis of the locust, and the z-axis is the anterior-posterior axis of the locust. It was important to not have the cameras facing the locust parallel to an $x, y$ or $z$ plane, they needed to be in multiple planes facing the locust to accurately acquire a 3-dimensional space. One camera was placed above the locust so it had a dorsal view, and the other below the locust for a ventral view at an angles of approximately $45^{\circ}$ to the y-axis. Both cameras were placed behind the locust and therefore each had a posterior view. The cameras were synchronized together by a manual trigger that would release the acoustic stimulus and record the same number of frames pre- and post-stimulus.

A spatial calibration of overlapping video fields of the two cameras was performed by using a custom-built calibration grid that had pre-measured visual markers 
on the surface of each layer of the grid. The grid had four layers measuring $1 \mathrm{~cm}$ apart so that measured distances were known in all three planes, $x, y$ and $z$. This calibration method allowed for the analysis of 3-dimensional points for measuring accurate kinematic variables.

Once the calibration grid was positioned in place, .TIFF images were captured from each of the cameras that showed the 40 point calibration grid from both cameras (Figure 2.5). Each of the 40 points of known displacement were used to establish the 3dimensional world coordinates by way of direct linear transformation.

Each trial was cut in length so that five to nine wing strokes pre-stimulus and five to nine wing strokes post-stimulus were analyzed to minimize the digitization process. The videos were imported into custom MatLab software (Hendrick, 2008), which employed a direct linear transform (DLT) to convert kinematic landmarks from each video into coordinates relative to the calibration (=locust flight axes). Forewing tips and bases as well as the hindwing tips and bases on both the left and right side of the locust were digitized frame by frame (Figure 2.6). Separate MatLab software (Dawson, unpublished) was used to translate the kinematic coordinates in to wing stroke amplitudes for each of the wings, separation angles and angular differences (Figure 2.7).

\subsubsection{Dye Flow visualization}

\subsubsection{The Spinner Tank}



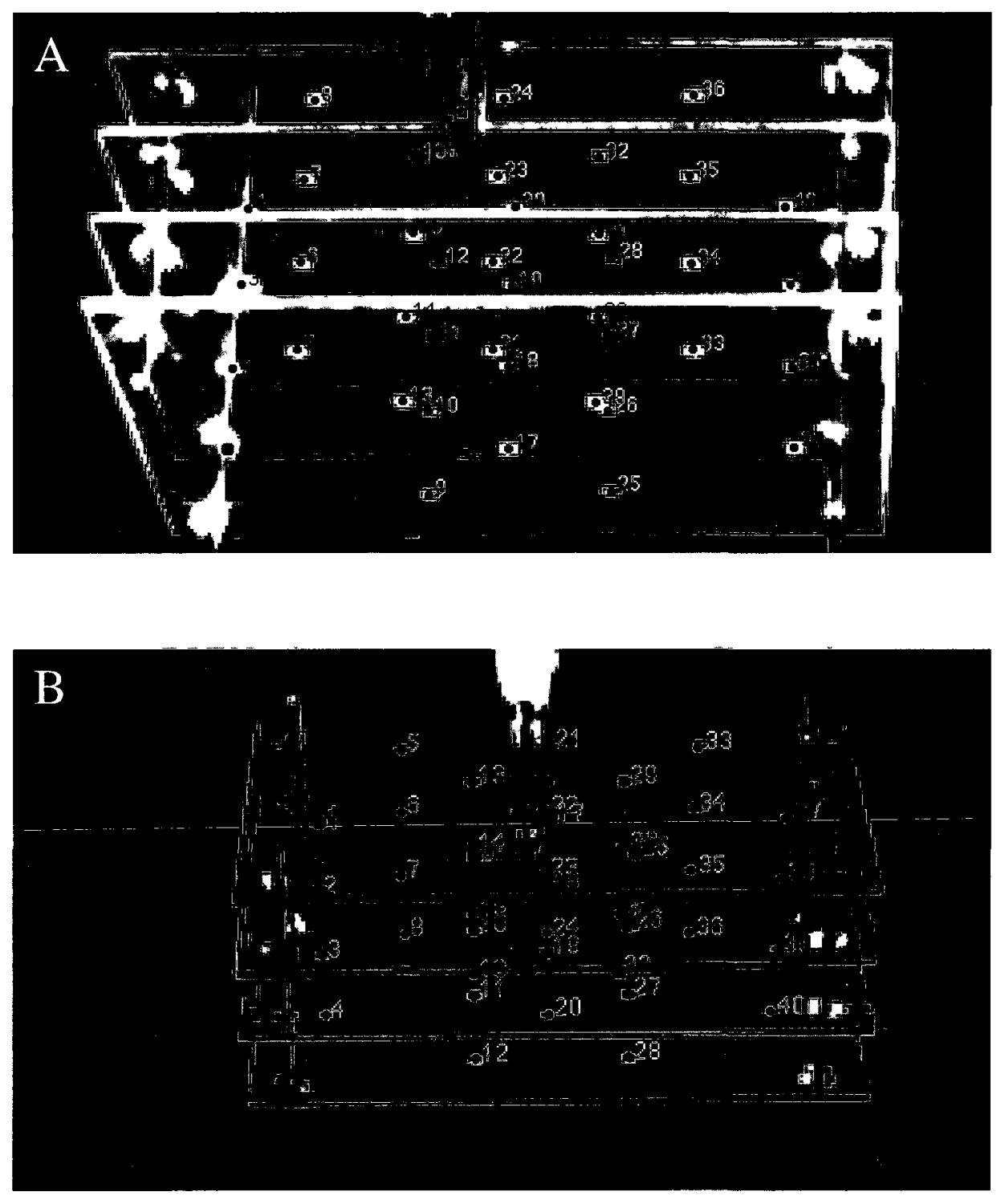

Figure 2.5. A 40-point calibration grid showing the view from the high-speed camera above (A) and from the other high-speed camera below (B) the working section of the wind tunnel. The calibration grid is placed in the same location as the locust would be during kinematic recordings. The grid coordinates determine the reference frame for the locust kinematics. The x-axis is the wing span of the locust, the y-axis is the dorsal-ventral axis of the locust and the z-axis is the anterior-posterior axis of the locust. 


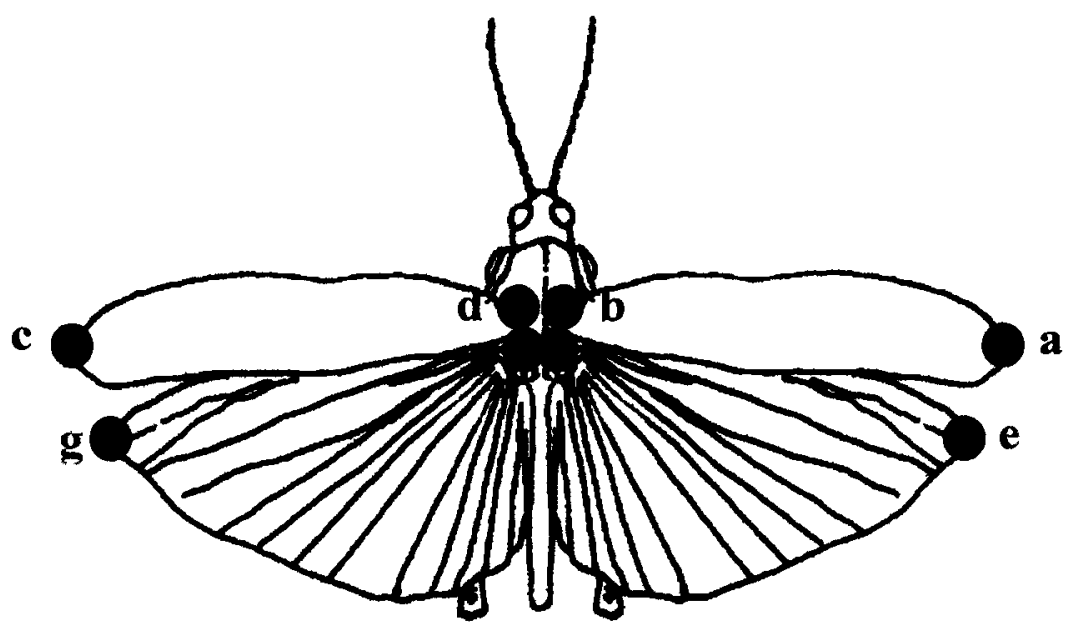

Figure 2.6. Schematic diagram of a tethered locust (dorsal view) illustrating the eight points digitized for kinematic reconstruction. The eight points consist of the right forewing tip (a) and base (b), the left forewing tip (c) and base (d), the right hindwing tip (e) and base (f), and finally the left hindwing tip (g) and base (h). 

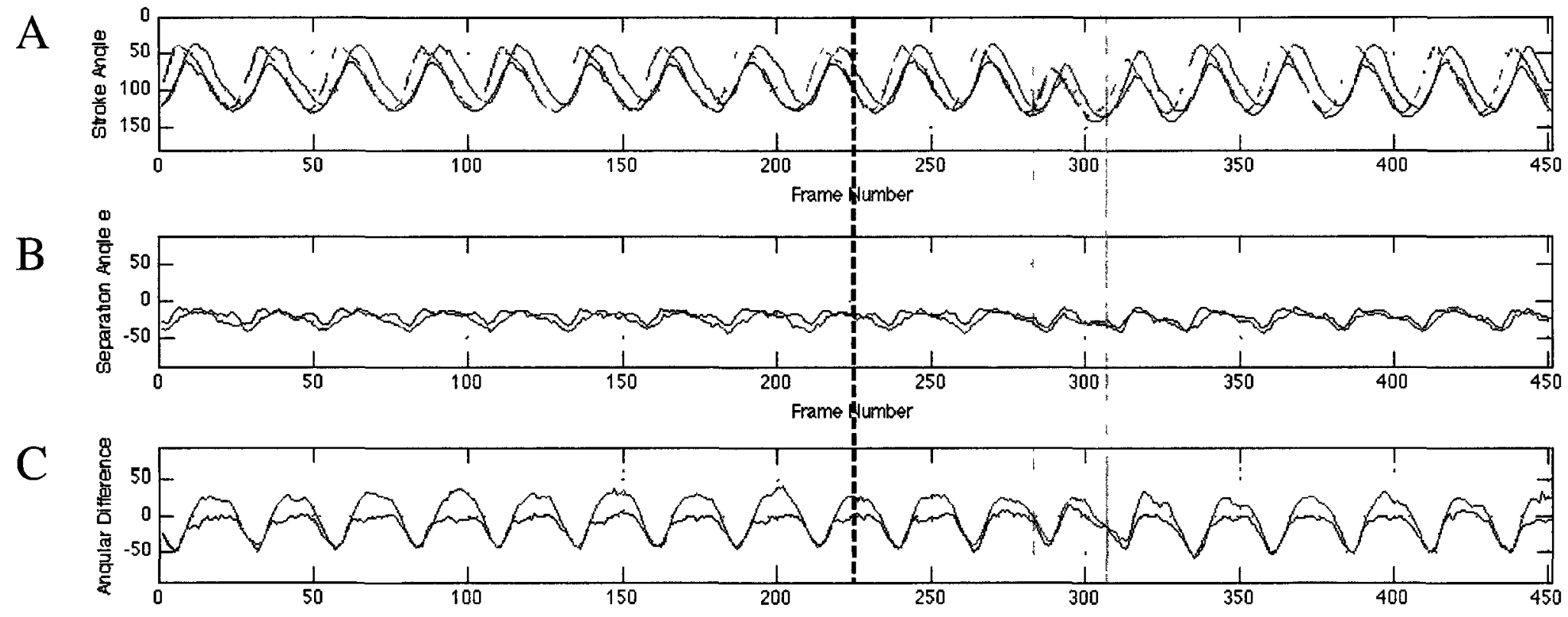

Figure 2.7. Kinematic results of a locust (09/24-2) in flight showing the wing stroke amplitude (A), separation angle (B) and angular difference $(\mathrm{C})$ of the left forewing (blue), right forewing (red), left hindwing (green) and right hindwing (yellow). The dashed red line indicates when the acoustic stimulus was introduced. The blue highlighted area shows the response of the locust to the acoustic stimulus (on the third stroke post-stimulus). The grey highlighted areas describe the downstroke from peak to peak of the right forewing and the corresponding separation angles and angular differences during that downstroke pre-stimulus and poststimulus respectively. 
The spinner tank places models in rotation about a central shaft and is useful for studying the downstroke phase of the wing beat. The spinner tank holds a capacity of 500 L of water (dimensions are indicated in Figure 2.2). Rotating models produce a gradient of chord-wise velocity from wing base to wingtip.

The model wings are rotated via a shaft attached to a motor unit system that uses a DC motor controlled by a variable speed controller. The speed can be adjusted to achieve the desired $\mathrm{Re}$ for the test of interest. An optical sensor was used to detect the rotation speed of the shaft. Variability in the speed of the motor occurred between trials. Given the different sizes and morphometric variability of individual locusts, a range of Reynolds numbers was established (termed biologically relevant range). Attention was paid to the variability of the speed of the motor so that it did not increase or decrease to a value that resulted in a Reynolds number beyond the biologically relevant range.

\subsubsection{Wing Models}

The length of the model wings was selected so that the wings rotating in water would match the Reynolds number of real locust wings operating in air using the following formulae:

$$
R e=\frac{4 \pi R^{2} f}{v A R}
$$

For a wing rotating in water, 


$$
R e=\frac{4 \varphi R^{2} f}{v A R}
$$

For a wing flapping in air with a stroke amplitude of $\varphi$

Where $\mathrm{R}$ is the wing length, $\mathrm{f}$ is the wing beat frequency, $v$ is the kinematic viscosity of air at $30^{\circ} \mathrm{C}$ or water at $25^{\circ} \mathrm{C}$ and $A R$ is the aspect ratio of the wing (Ellington, 1999). Matching the Reynolds number for wings under water yielded a rotation speed of $11.8 \mathrm{~s} \pm$ $0.7 \mathrm{~s}$ was selected for use in the spinner tank resulting in a forewing length of $15 \mathrm{~cm}$ and a hindwing length of $13.5 \mathrm{~cm}$.

Wing models were constructed using $1.6 \mathrm{~mm}$ thick polystyrene plastic. A outline of real locust fore- and hindwings were proportionally scaled, traced on to the plastic, cut out using a dremmel tool (Mastercraft, model 54-4703-6) and hand filed so that the edges of the wings met at a $45^{\circ}$ angle. Wings were attached to the spinner shaft using custom designed and fabricated chucks that allow for the adjustment of angle of attack, separation angle and angular difference along the shaft of the rotating motor. The angle of attack, angular difference and separation angles of the wings were adjusted using a protractor to achieve the desired angle (Figure 2.8).

Malachite green dye crystals were placed at the base of both the fore- and hindwing model to reveal any leading-edge vortex (LEV) formation. The dye becomes entrained in areas of low pressure formed by the rotating wings. The LEV structures are areas of low pressure. A high-definition digital still camera (Casio Ex-F1) was used to capture the flow patterns of the model in the spinner tank. A trial consisted between 5 to 

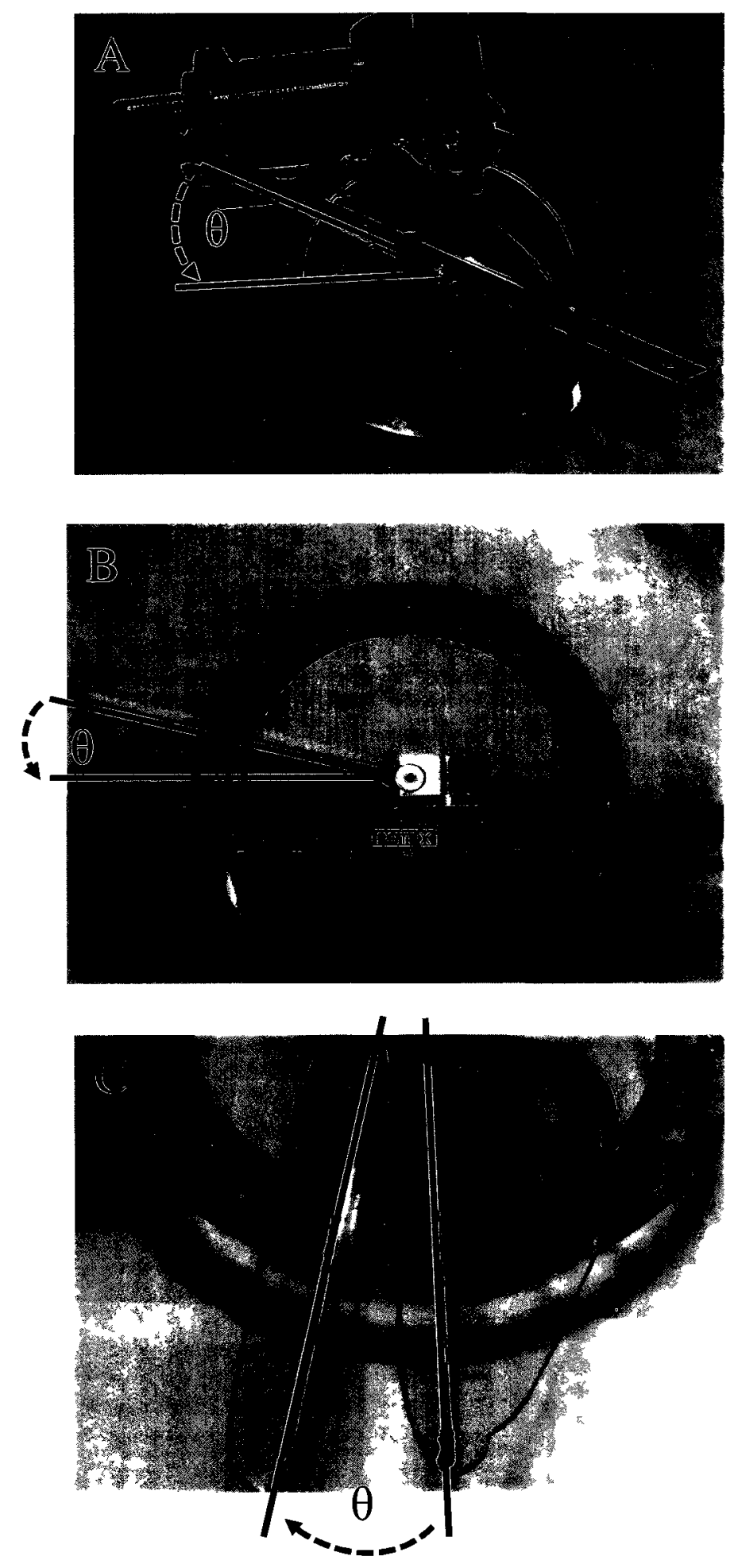

Figure 2.8. Photographs showing how the angle of attack (A), angular difference (B) and separation angle $(\mathrm{C})$ was set for the wing models. 
10 rotations, where each rotation was filmed. From the videos, .JPEG still images were taken from the videos of several wing configurations illustrating the LEV formations, detachment points and flow structures as a result of wing-wing interactions.

\subsubsection{Volumetric 3-Component Velocimetry}

\subsubsection{System Calibration and Tank Set-Up}

The V3V system measures the displacement of particles that are seeded in a fluid, in this case water. The system includes a 3-dimensional camera (model V3V-8000), a TSI LaserPulse (model YAG 200-NW), hyperstreaming image transfer and computer system, and INSIGHT V3V software (C TSI Inc., 2008).

A volume of fluid measuring $140 \times 140 \times 100 \mathrm{~mm}$ is illuminated by a cone of laser light. The camera is first calibrated by placing a calibration grid in the cone of laser light and the coordinates are then translated as a quantifiable 3-dimensional volumetric space using the INSIGHT V3V software. The spinner shaft is placed so that the wings directly enter this volumetric space, which is centered in front of the camera during calibration. The volumetric area captured in the $\mathrm{V} 3 \mathrm{~V}$ analyses was not large enough to capture the entire wing structures from base to tip, it was only able to cover approximately $70 \%$ of the wing length from the tip, lacking the additional $30 \%$ to the base of the wings. As the wings rotate in front of the camera, a manual trigger signals the laser to emit two pulses of high intensity laser light that are separated by a short duration called delta t. This laser light is reflected off the particles so that the camera can distinguish the particles from the 
fluid and capture their position. The delta $t$ is adjustable depending on the rotation speed of the wings. The camera captures the two images, one for each pulse and using a 3aperture imaging technique, the position of all the particles for each laser pulse is determined. Once the images are transferred to the computer system, the INSIGHT V3V software processes the image to track the movement of each particle between the delta $t$ to an accuracy of 10 microns in the $\mathrm{x}, \mathrm{y}$ plane, and 100 microns in the $\mathrm{z}$ plane (TSI Inc.). Once the images are processed, advanced software tools allow for the quantifiable measurements such as particle velocity, direction, vorticity magnitude, and rotational vorticity direction involved in flow structures, etc. Options such as plots, graphics and movie generation of flow are also available in the advanced software tools, which allows for integrated 3-dimensional graphics and representation of flow over the gross structure of the wings.

Specifically for this experiment, a glass tank measuring $400 \times 80 \times 80 \mathrm{~cm}$ was filled with water and was seeded with particles measuring approximately 65 microns (Figure 2.9). This tank was used in lieu of the spinner tank as this part of the experiment needed to be conducted in a laser safe room. The same spinner shaft used with varying wingwing configurations for the dye-flow visualization experiment was placed with the wings in the centre of the field of view from all three apertures; however, the Malachite green dye crystals were not required for visualization. In order to achieve a Reynolds number of 2800 for the forewing, a rotation speed of $11.8 \mathrm{~s}$ was used.

A manual trigger was used to signal the laser to fire, which in turn signaled the camera to capture the images. A trial consisted of 40 rotations and any number of trials would be completed for every wing-wing configuration until yields of 18-20 usable 


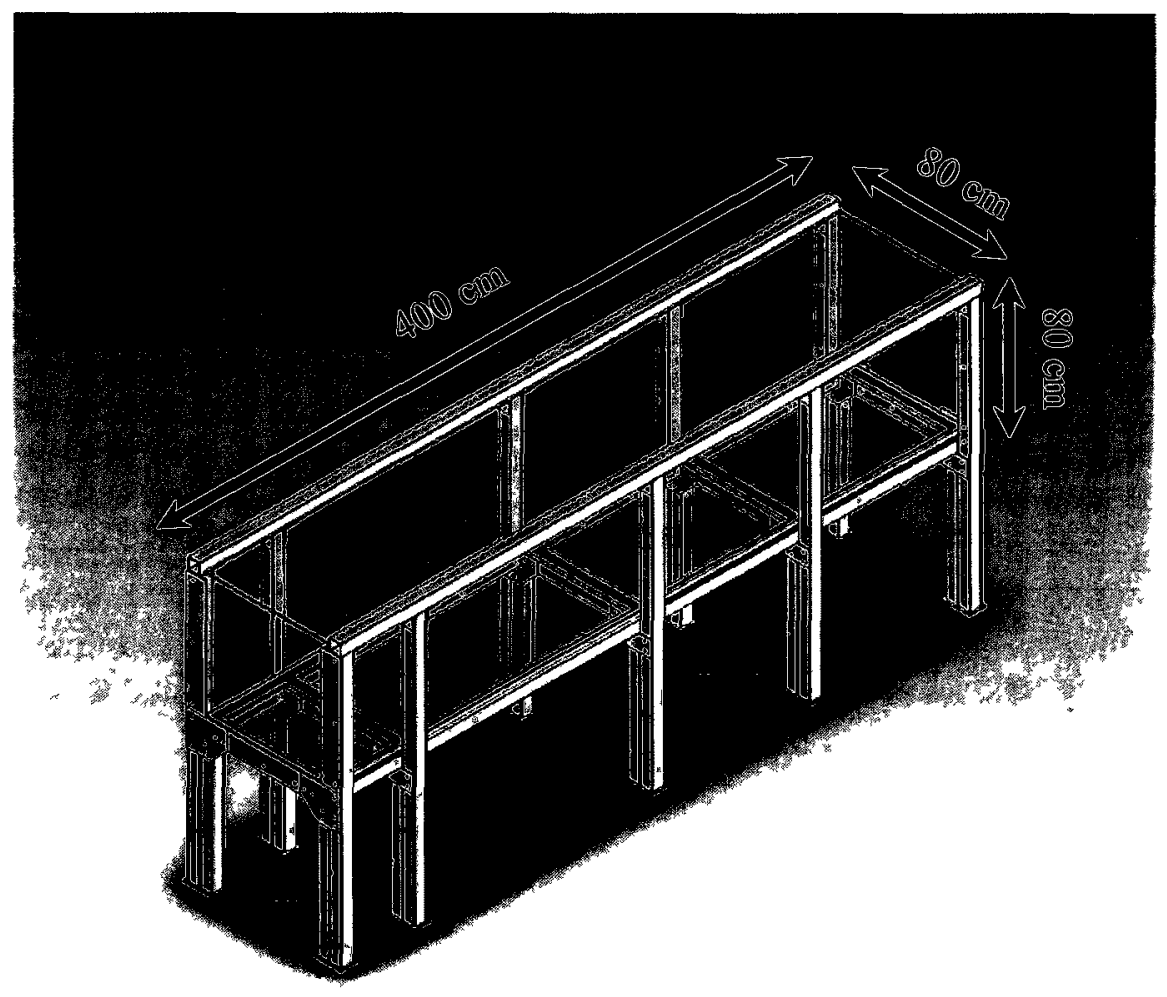

Figure 2.9. Diagram illustrating the dimensions of the tank used for V3V measurements. The spinner was placed at approximately $1 / 3^{\text {rd }}$ the length of the tank for V3V measurements. 
(meaning wings were facing end-on with the camera and located at the same position as the other rotations) images were produced. Only the usable images were then processed and analyzed using the INSIGHT V3V software.

\subsubsection{Post Processing and Analysis}

After the images have been processed using the INSIGHT V3V software, they were imported into a visualization folder for post-processing and analysis. Variables and thresholds were added and adjusted to see the desired flow structures surrounding the wing configurations. The variables included vector direction and magnitude as well as vorticity direction and magnitude, which in a 3-dimensional plane, was represented by isotructures varying in size depending on the magnitude, and when viewed in a 2dimensional plane was represented by a colour gradient showing velocity magnitude. Sheets could be inserted along any of the three planes, $\mathrm{x}, \mathrm{y}$ or $\mathrm{z}$ to isolate that area in a 2dimensional way for further analysis. For example, a sheet was inserted at the approximate detachment point of the LEV structure on the hindwing to examine the vorticity magnitude at that specific location. When the desired images and thresholds were obtained for each configuration, .JPEG images were saved to represent the data collected. 


\subsection{Results}

\subsubsection{Kinematics}

\subsubsection{Tethering Effects}

The two different tethering methods, the semi-rigid ventral tether and rigid sternal tether, were compared to see if there were any differences in the flight kinematics. Wing beat frequency is a good indicator of tethering effects as it has previously been stated the tethers interfere with the lift production of a locust (Kutsch, 1999). Since wing beat frequency is directly related to in the production of lift, then significant differences in wing beat frequencies across trials may indicate tethering artefacts. A previous study conducted (Fauquier, 2008), showed statistical differences among several tethering methods across varying kinematic parameters, wing beat frequency being one variable sensitive to the tethering methods. I compared wing beat frequency of tethered flying locusts before and after a stimulus was applied between the locusts secured with the semi-rigid ventral tether $(\mathrm{N}=4)$ and locusts tethered with the rigid sternal tether $(\mathrm{N}=10)$ (Table 2.2). Wing beat frequency was not significantly different between locusts tethered with the two tethering methods ( $\mathrm{t}$-test, $\mathrm{t}=1.156, \mathrm{n}=4,10, \mathrm{p}=0.2703$ ) (Figure 2.10).

Due to the fact that there are no significant differences between the two different tethering methods, the data sets were combined for the remainder of the kinematic analyses 


\subsubsection{Steering (Pre vs. Post-Stimulus)}

The locust acoustic startle response (ASR) (Robert, 1989) is an evasive maneuver that is hypothesized to function in predator avoidance, specifically the avoidance of echolocating insectivorous bats. Robert (1989) showed that the ASR is comprised of changes in WBF (including flight cessation), abdomen deflection and dorsiflection, head rolling and hind leg extension. These changes presumably allow the insect to steer, dive or even possibly climb rapidly away from the predator. Two-tailed paired t-tests were conducted on wing stroke amplitude, forewing asymmetry, separation angle and angular difference to determine if any significant differences existed pre-stimulus relative to post-stimulus.

\subsubsection{Wing Beat Frequency}

Although locusts did not show a significant difference in wing beat frequency (WBF) between the two tethering methods, there was a significant change in WBF from pre- to post-stimulus $(\mathrm{t}=-2.725, \mathrm{n}=14, \mathrm{p}=0.0173)$. The majority of locusts showed an increase in WBF, but three locusts showed a decrease in WBF (Figure 2.10, Table 2.2).

\subsubsection{Wing Stroke Amplitude}

No significant differences were found in wing stroke amplitude when the ipsilateral and contralateral forewings (relative to the inferred steering direction - Figure 
Table 2.2. Summary of calculated change in wing beat frequencies and forewing asymmetries post-stimulus. Results are averaged over 5-10 wing strokes and forewing asymmetries are determined at $1 / 3$ the downstroke.

\begin{tabular}{|c|c|c|c|c|}
\hline \multirow[b]{2}{*}{ Locust } & \multicolumn{2}{|c|}{ Wing Beat Frequency (WBF) (Hz) } & \multicolumn{2}{|c|}{ Forewing Asymmetry (FWA) } \\
\hline & Change in WBF (Hz) & Increase or Decrease & Change in FWA $\left(^{\circ}\right)$ & $\begin{array}{l}\text { Inferred Direction } \\
\text { of Turn* }\end{array}$ \\
\hline $09 / 24-1$ & 1.2494 & INCREASE & -1.63 & LEFT \\
\hline $09 / 24-2$ & 0.4329 & INCREASE & -3.92 & LEFT \\
\hline $12 / 11-1$ & 0.8735 & INCREASE & 1.28 & RIGHT \\
\hline $12 / 11-2$ & 1.4380 & INCREASE & -0.05 & LEFT \\
\hline $01 / 11-1$ & 1.1704 & INCREASE & -9.18 & LEFT \\
\hline $01 / 11-2$ & 0.0320 & INCREASE & -3.27 & LEFT \\
\hline $01 / 11-3$ & 1.3495 & INCREASE & 1.67 & RIGHT \\
\hline $01 / 11-4$ & -0.2083 & DECREASE & 2.97 & RIGHT \\
\hline $01 / 23-1$ & 0.6808 & INCREASE & -2.21 & LEFT \\
\hline $01 / 23-2$ & 1.0113 & INCREASE & -0.48 & LEFT \\
\hline $10 / 11-1$ & 1.6359 & INCREASE & -7.62 & LEFT \\
\hline $10 / 11-2$ & -0.4252 & DECREASE & -3.71 & LEFT \\
\hline $10 / 11-3$ & -1.2499 & DECREASE & 3.06 & RIGHT \\
\hline $10 / 11-4$ & 0.4202 & INCREASE & 2.37 & RIGHT \\
\hline Average & 0.6008 & & -1.48 & \\
\hline St. dev. & 0.824810903 & & 3.796590205 & \\
\hline
\end{tabular}

*See Dawson et. al., 1997, Dawson et. al., 2001 


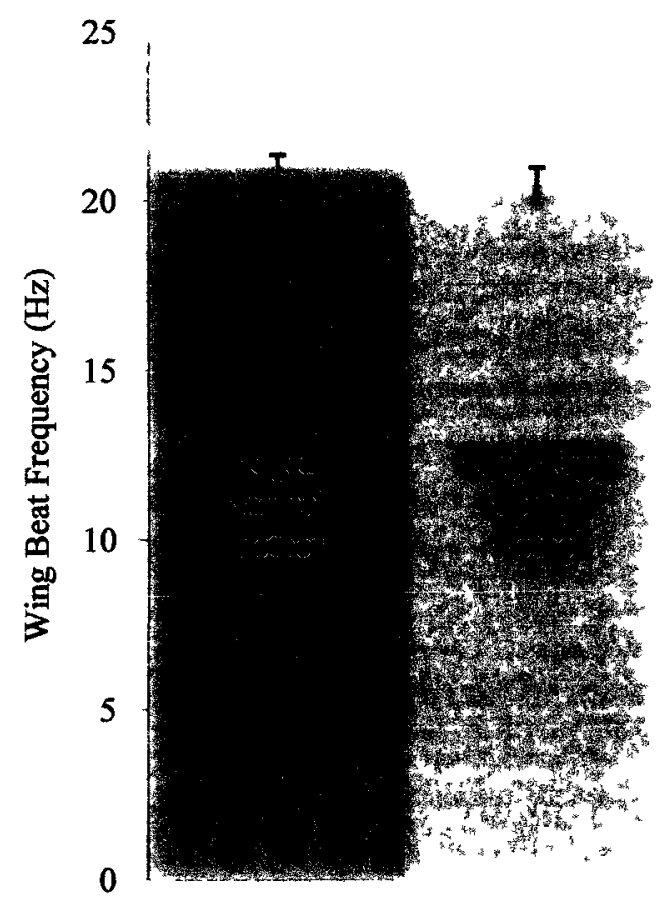

Pre-Stimulus

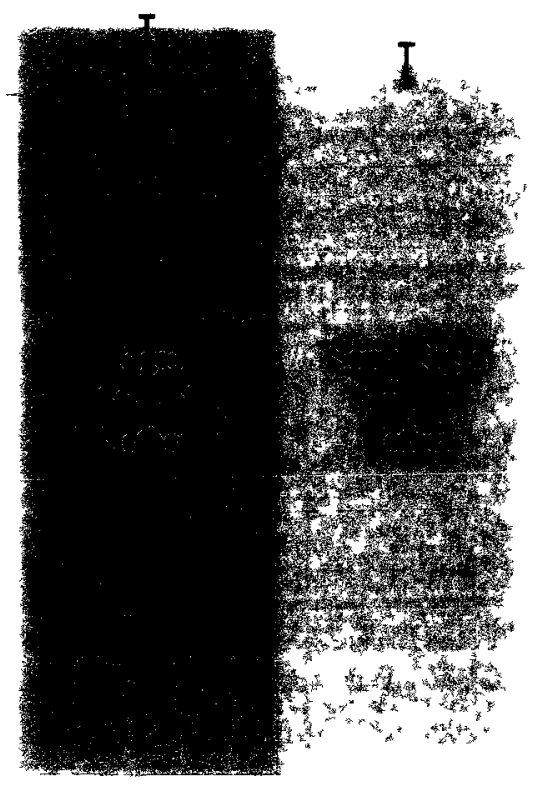

Post-Stimulus

Figure 2.10. The average wing beat frequencies of all locusts tethered with the rigid sternal tether pre- and post-stimulus, and the semi-rigid ventral tether pre- and post-stimulus. There were no significant differences in wing beat frequencies between the two tethering methods ( $t$-test $t=1.156, n=4,10, p=0.2703$ ); however, there was a significant difference in wing beat frequency for all locust trials from pre- to post-stimulus $(-2.725, \mathrm{~N}=14, \mathrm{P}=0.0173)$. Standard error is represented by error bars. 
1.5) were compared before and after the stimulus was applied $(t=0.287, n=14, p=0.7784)$. Wing stroke amplitude was an average of $70.49^{\circ} \pm 11.01^{\circ}\left(\max : 90.82^{\circ}, \min : 62.49^{\circ}\right)$. A significant difference was found when the pre-stimulus ipslateral and contralateral hindwings were compared $(t=2.296, n=14, p=0.0390)$ with an average of $81.81^{\circ} \pm 9.50^{\circ}\left(\max : 118.85^{\circ}, \min : 62.49^{\circ}\right)$; however, when a single value (Locust $01 / 11-$ 4) was removed, the maximum became $92.93^{\circ}$ and there was no significant difference, indicating that the difference was most likely a spurious result due to an outlier (Figure 2.13, Table 2.3). There were no significant differences between the post-stimulus ipsilateral and contralateral hindwings were compared $(t=1.991, n=14, p=0.0679)$ with an average of $83.92^{\circ} \pm 9.12^{\circ}\left(\max : 119.88^{\circ}\right.$, $\left.\min : 67.90^{\circ}\right)$.

Next, I compared the ipsilateral and contralateral forewings pre- to post-stimulus to see if changes occurred independently from each other. Both showed a significant increase from pre-to post-stimulus, ipsi $(t=-3.133, n=14, p=0.0079)$ with an average of $74.60^{\circ} \pm 11.26^{\circ}\left(\max : 99.50^{\circ}, \min : 60.62^{\circ}\right)$ post stimulus, and contra $(t=-4.461, n=14$, $\mathrm{p}=0.0006)$ with an average of $74.49^{\circ} \pm 11.54^{\circ},\left(\max : 91.90^{\circ}\right.$, min: $\left.56.31^{\circ}\right)$ post stimulus. Although both ipsilateral and contralateral forewings increased in wing stroke amplitude after the stimulus was applied, they were not significantly different from each other $(\mathrm{t}=0.038, \mathrm{n}=14, \mathrm{p}=0.9702)$ (Figures 2.11 and 2.12).

Neither the ipsilateral nor the contralateral hindwings showed any significant differences in WSA pre- to post-stimulus, ipsi $(t=-1.936, n=14, p=0.0749)$ with an average of $86.70^{\circ} \pm 11.40^{\circ},\left(\max : 119.88^{\circ}, \min : 74.27^{\circ}\right)$ post-stimulus, and contra $(t=1.985, n=14, p=0.0686)$ with an average of $80.06^{\circ} \pm 7.21^{\circ},\left(\max : 90.87^{\circ}, \min : 67.90^{\circ}\right)$ post-stimulus (Figures 2.13 and 2.14). 
Table 2.3. Summary of wing stroke amplitude measurements during kinematic trials

\begin{tabular}{|c|c|c|c|c|c|c|c|c|}
\hline \multirow[b]{2}{*}{ Locust } & \multirow[b]{2}{*}{ Wing } & \multicolumn{7}{|c|}{ Wing Stroke Amplitude - WSA $\left(^{\circ}\right)$} \\
\hline & & Mean & Median & $\begin{array}{c}\text { Standard } \\
\text { Deviation }\end{array}$ & $\begin{array}{c}\text { MAX Pre- } \\
\text { Stimulus }\end{array}$ & $\begin{array}{c}\text { MAX Post- } \\
\text { Stimulus }\end{array}$ & $\begin{array}{l}\text { MIN Pre- } \\
\text { Stimulus }\end{array}$ & $\begin{array}{c}\text { MIN Post- } \\
\text { Stimulus }\end{array}$ \\
\hline $09 / 24-1$ & \multirow{14}{*}{ LFW } & 81.39 & 80.68 & \multirow{14}{*}{11.79159} & 83.30 & 88.73 & 75.96 & 75.69 \\
\hline $09 / 24-2$ & & 94.69 & 94.30 & & 100.53 & 111.79 & 63.35 & 90.34 \\
\hline $12 / 11-1$ & & 91.36 & 91.29 & & 95.30 & 94.10 & 86.99 & 90.20 \\
\hline $12 / 11-2$ & & 65.94 & 66.15 & & 66.89 & 73.67 & 61.63 & 59.90 \\
\hline $01 / 11-1$ & & 67.01 & 66.86 & & 66.08 & 78.46 & 56.32 & 67.63 \\
\hline $01 / 11-2$ & & 64.47 & 64.11 & & 68.72 & 69.78 & 60.32 & 58.24 \\
\hline $01 / 11-3$ & & 70.71 & 68.08 & & 68.51 & 83.39 & 58.82 & 67.19 \\
\hline $01 / 11-4$ & & 61.03 & 60.74 & & 62.75 & 64.52 & 57.70 & 59.34 \\
\hline $01 / 23-1$ & & 67.06 & 67.10 & & 74.12 & 73.31 & 62.84 & 58.11 \\
\hline $01 / 23-2$ & & 64.97 & 62.87 & & 70.22 & 71.85 & 59.77 & 62.05 \\
\hline $10 / 11-1$ & & 61.07 & 59.64 & & 78.71 & 64.69 & 57.41 & 57.10 \\
\hline $10 / 11-2$ & & 73.68 & 73.57 & & 74.91 & 76.45 & 70.94 & 72.97 \\
\hline $10 / 11-3$ & & 56.67 & 57.83 & & 57.94 & 63.40 & 47.45 & 57.83 \\
\hline $10 / 11-4$ & & 56.94 & 55.21 & & 61.36 & 66.37 & 51.48 & 52.22 \\
\hline $09 / 24-1$ & \multirow{14}{*}{ RFW } & 84.81 & 84.72 & \multirow{14}{*}{9.713368} & 87.29 & 91.84 & 77.19 & 82.85 \\
\hline $09 / 24-2$ & & 79.12 & 80.53 & & 85.58 & 90.22 & 35.90 & 76.03 \\
\hline $12 / 11-1$ & & 84.90 & 84.02 & & 93.61 & 92.69 & 71.56 & 83.72 \\
\hline $12 / 11-2$ & & 62.66 & 61.80 & & 61.80 & 69.12 & 56.63 & 64.37 \\
\hline $01 / 11-1$ & & 79.01 & 76.61 & & 79.82 & 94.99 & 66.93 & 72.83 \\
\hline $01 / 11-2$ & & 80.73 & 82.59 & & 86.02 & 86.77 & 74.06 & 71.66 \\
\hline $01 / 11-3$ & & 86.30 & 84.34 & & 85.50 & 98.07 & 79.98 & 83.44 \\
\hline $01 / 11-4$ & & 73.17 & 73.45 & & 77.72 & 78.12 & 70.42 & 68.60 \\
\hline $01 / 23-1$ & & 80.61 & 80.85 & & 89.83 & 85.80 & 73.85 & 71.07 \\
\hline $01 / 23-2$ & & 69.81 & 70.79 & & 82.16 & 77.09 & 61.14 & 69.91 \\
\hline $10 / 11-1$ & & 54.65 & 52.52 & & 71.50 & 60.89 & 48.36 & 51.58 \\
\hline $10 / 11-2$ & & 82.72 & 82.45 & & 83.63 & 89.56 & 79.20 & 80.91 \\
\hline $10 / 11-3$ & & 69.21 & 65.86 & & 65.86 & 76.83 & 61.02 & 73.00 \\
\hline $10 / 11-4$ & & 64.16 & 63.51 & & 69.86 & 70.05 & 62.19 & 60.61 \\
\hline
\end{tabular}




\begin{tabular}{|c|c|c|c|c|c|c|c|c|}
\hline $09 / 24-1$ & & 82.83 & 82.85 & & 84.89 & 87.85 & 79.69 & 82.14 \\
\hline $09 / 24-2$ & & 98.01 & 97.63 & & 102.92 & 109.86 & 75.32 & 96.19 \\
\hline $12 / 11-1$ & & 73.40 & 73.86 & & 78.77 & 76.45 & 71.20 & 67.21 \\
\hline $12 / 11-2$ & & 81.51 & 81.64 & & 81.64 & 90.90 & 75.78 & 81.68 \\
\hline $01 / 11-1$ & & 80.58 & 80.40 & & 79.81 & 85.85 & 74.87 & 80.99 \\
\hline $01 / 11-2$ & & 88.02 & 89.37 & & 95.95 & 93.17 & 87.49 & 70.48 \\
\hline $01 / 11-3$ & \multirow{8}{*}{ LHW } & 80.22 & 78.13 & 6.909986 & 78.23 & 86.52 & 74.36 & 78.03 \\
\hline $01 / 11-4^{*}$ & & 91.00 & 91.03 & & 94.53 & 94.47 & 87.91 & 88.28 \\
\hline $01 / 23-1$ & & 84.23 & 86.48 & & 90.90 & 92.38 & 82.84 & 63.67 \\
\hline $01 / 23-2$ & & 82.37 & 81.80 & & 86.84 & 88.42 & 78.23 & 79.25 \\
\hline $10 / 11-1$ & & 73.60 & 73.17 & & 77.39 & 77.02 & 70.77 & 71.47 \\
\hline $10 / 11-2$ & & 81.77 & 81.73 & & 84.80 & 85.21 & 77.40 & 79.59 \\
\hline $10 / 11-3$ & & 73.28 & 74.45 & & 75.68 & 78.19 & 67.63 & 74.45 \\
\hline $10 / 11-4$ & & 78.08 & 78.40 & & 80.95 & 81.45 & 76.95 & 68.73 \\
\hline $09 / 24-1$ & \multirow{14}{*}{ RHW } & 87.97 & 88.20 & \multirow{14}{*}{11.9336} & 90.06 & 94.19 & 83.58 & 84.30 \\
\hline $09 / 24-2$ & & 81.88 & 83.87 & & 88.00 & 89.01 & 46.05 & 83.65 \\
\hline $12 / 11-1$ & & 87.01 & 87.05 & & 91.63 & 90.67 & 83.06 & 80.94 \\
\hline $12 / 11-2$ & & 64.95 & 65.46 & & 71.93 & 73.91 & 57.98 & 65.46 \\
\hline $01 / 11-1$ & & 77.29 & 77.47 & & 78.17 & 88.96 & 69.60 & 77.32 \\
\hline $01 / 11-2$ & & 87.43 & 89.22 & & 94.02 & 93.63 & 84.61 & 72.14 \\
\hline $01 / 11-3$ & & 78.11 & 77.69 & & 78.53 & 81.82 & 73.87 & 77.25 \\
\hline $01 / 11-4^{*}$ & & 119.37 & 119.28 & & 120.50 & 121.98 & 117.67 & 118.06 \\
\hline $01 / 23-1$ & & 82.74 & 84.06 & & 87.47 & 89.55 & 80.98 & 66.90 \\
\hline $01 / 23-2$ & & 79.31 & 78.98 & & 83.89 & 82.62 & 75.16 & 77.51 \\
\hline $10 / 11-1$ & & 76.09 & 75.75 & & 78.95 & 81.11 & 71.85 & 73.99 \\
\hline $10 / 11-2$ & & 86.46 & 85.79 & & 85.94 & 93.45 & 82.28 & 85.63 \\
\hline $10 / 11-3$ & & 82.02 & 83.19 & & 83.21 & 89.88 & 75.47 & 79.59 \\
\hline $10 / 11-4$ & & 79.15 & 78.97 & & 83.44 & 83.77 & 77.98 & 73.31 \\
\hline Average & & 77.63 & 77.47 & \multirow[t]{2}{*}{10.08714} & 81.40 & 84.37 & 70.54 & 73.89 \\
\hline St. dev. & & 11.46 & 11.80 & & 11.45 & 11.93 & 13.21 & 11.69 \\
\hline
\end{tabular}

*outlier which suggests a spurious result 
A

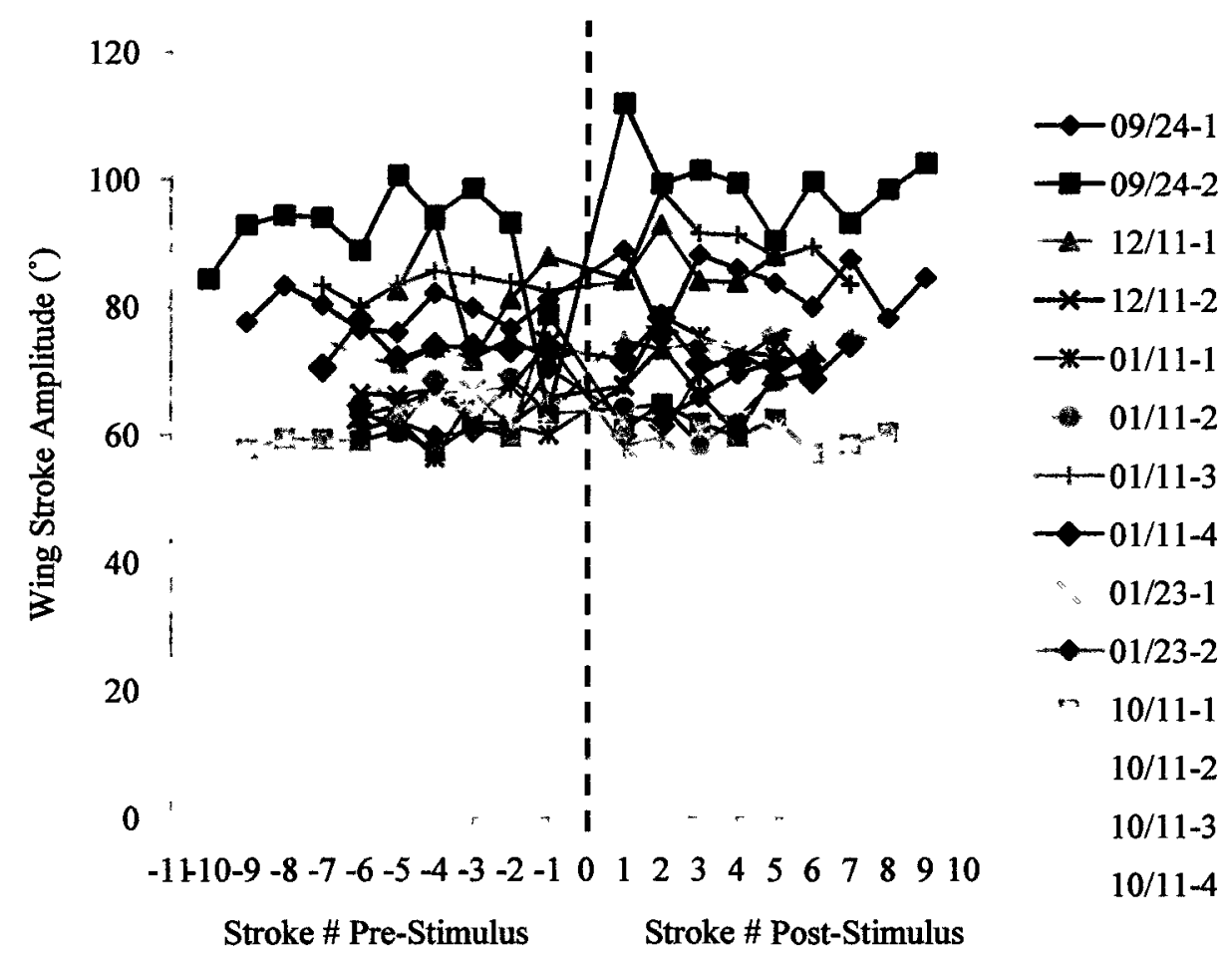

B

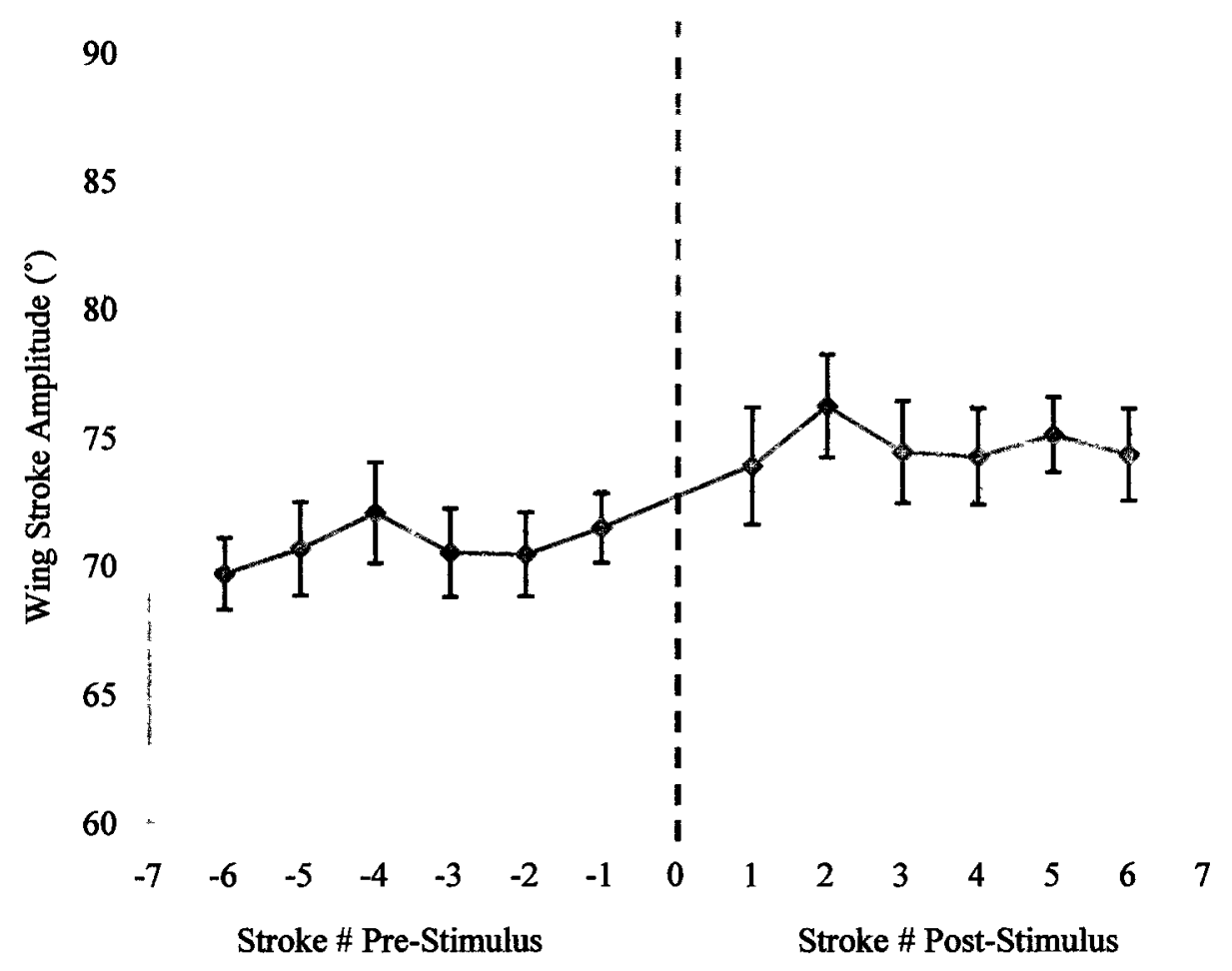

Figure 2.11. Wing stroke amplitudes of the ipsilateral forewings of all locusts (A) and the average (B). Dashed red line indicates when the acoustic stimulus was introduced. There was a significant increase in wing stroke amplitude from pre- to post-stimulus $(t=-3.133, n=14, p=0.0079)$. Standard error is represented by error bars. 
A

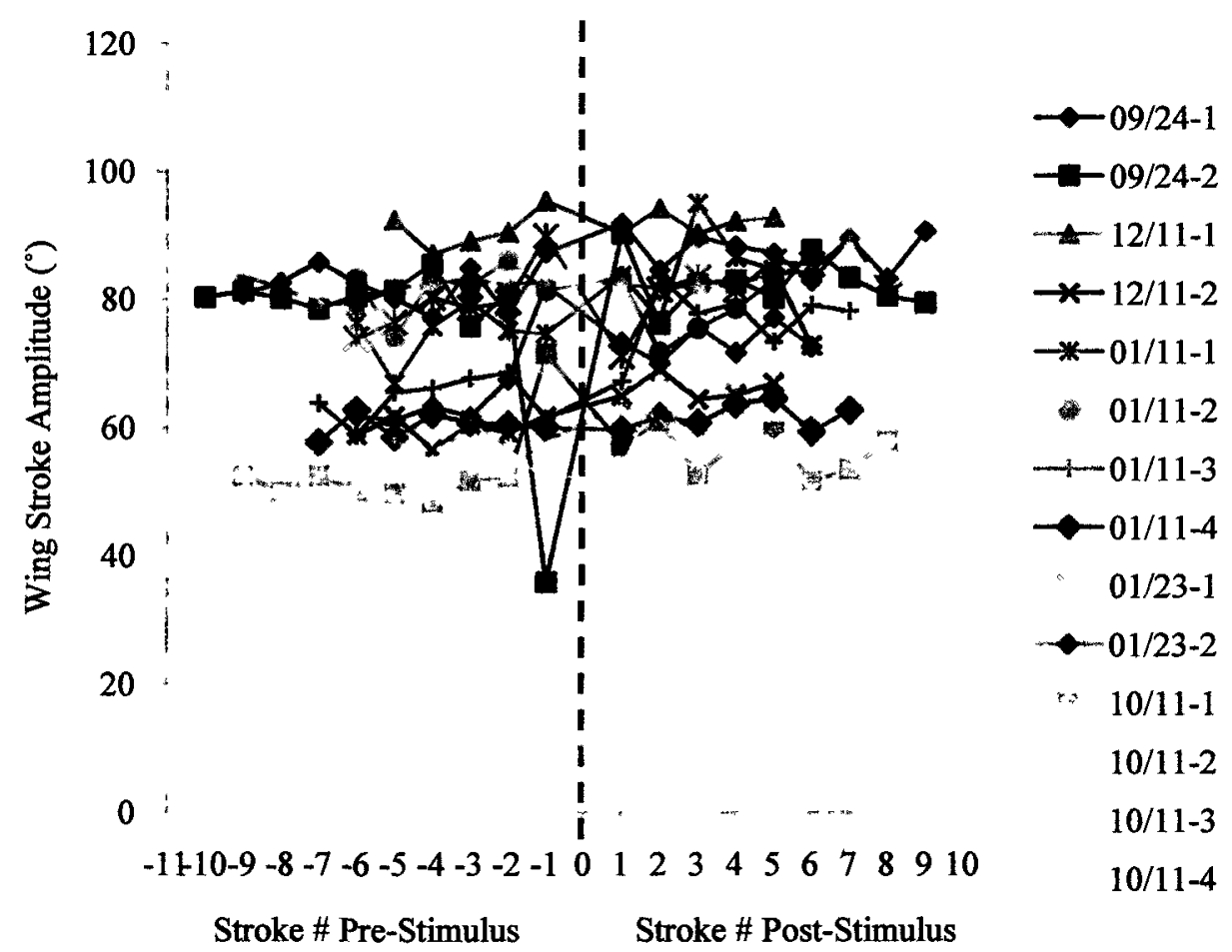

B

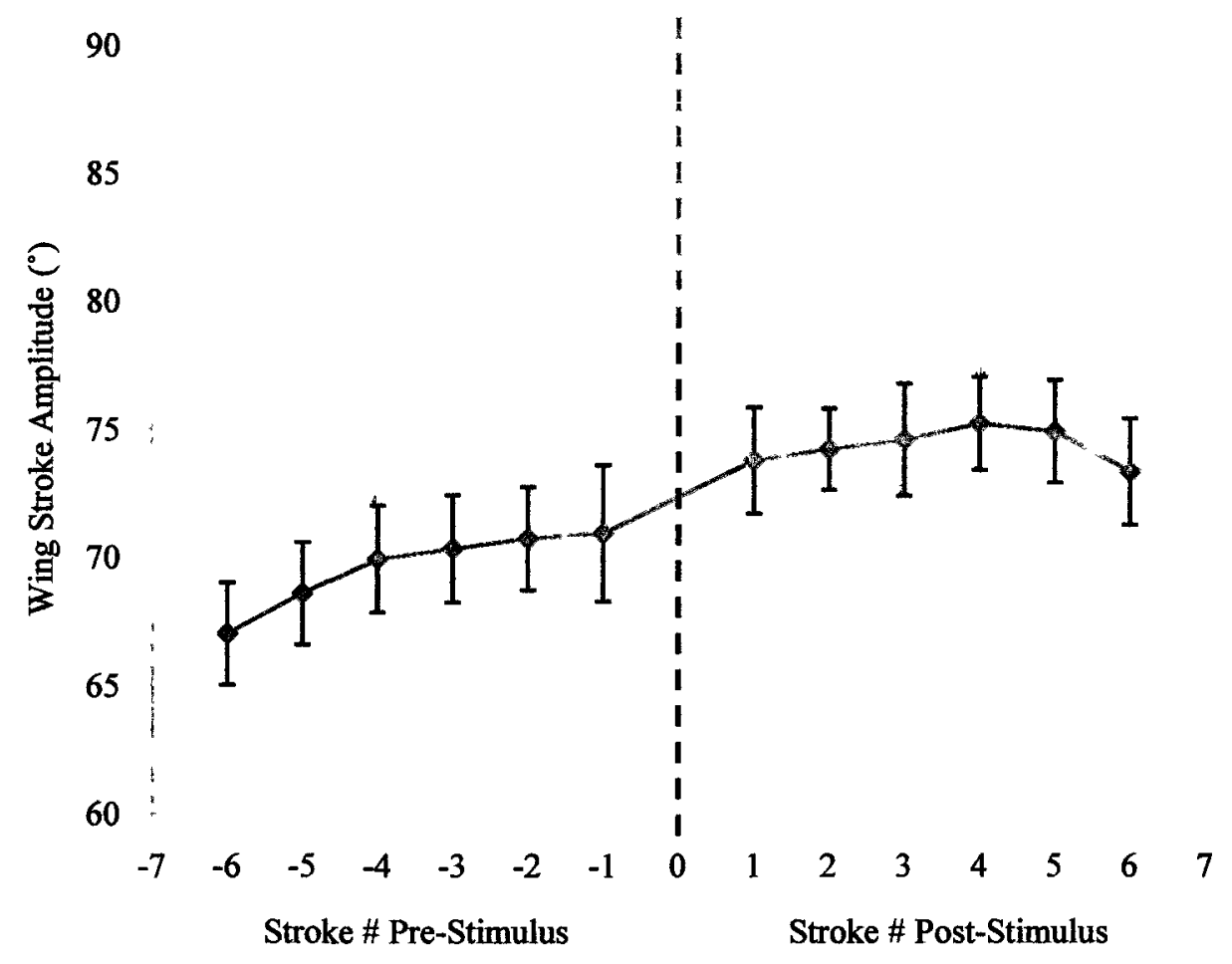

Figure 2.12. Wing stroke amplitudes of the contralateral forewings of all locusts (A) and the average (B). Dashed red line indicates when the acoustic stimulus was introduced. There was a significant increase in wing stroke amplitude from pre- to post-stimulus $(\mathrm{t}=-4.461, \mathrm{n}=14, \mathrm{p}=0.0006)$. Standard error is represented by error bars. 
A

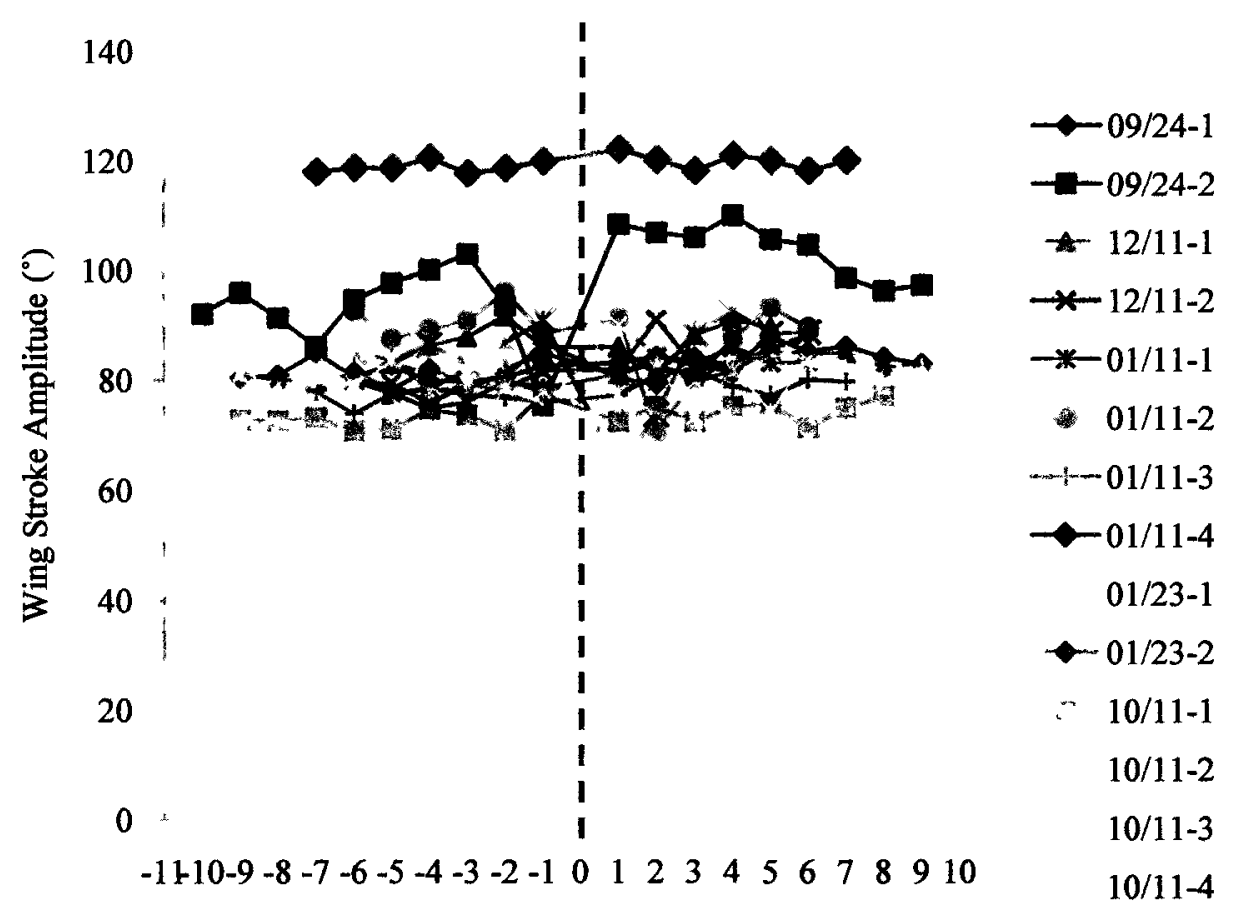

Stroke \# Pre-Stimulus Stroke \# Post-Stimulus

B

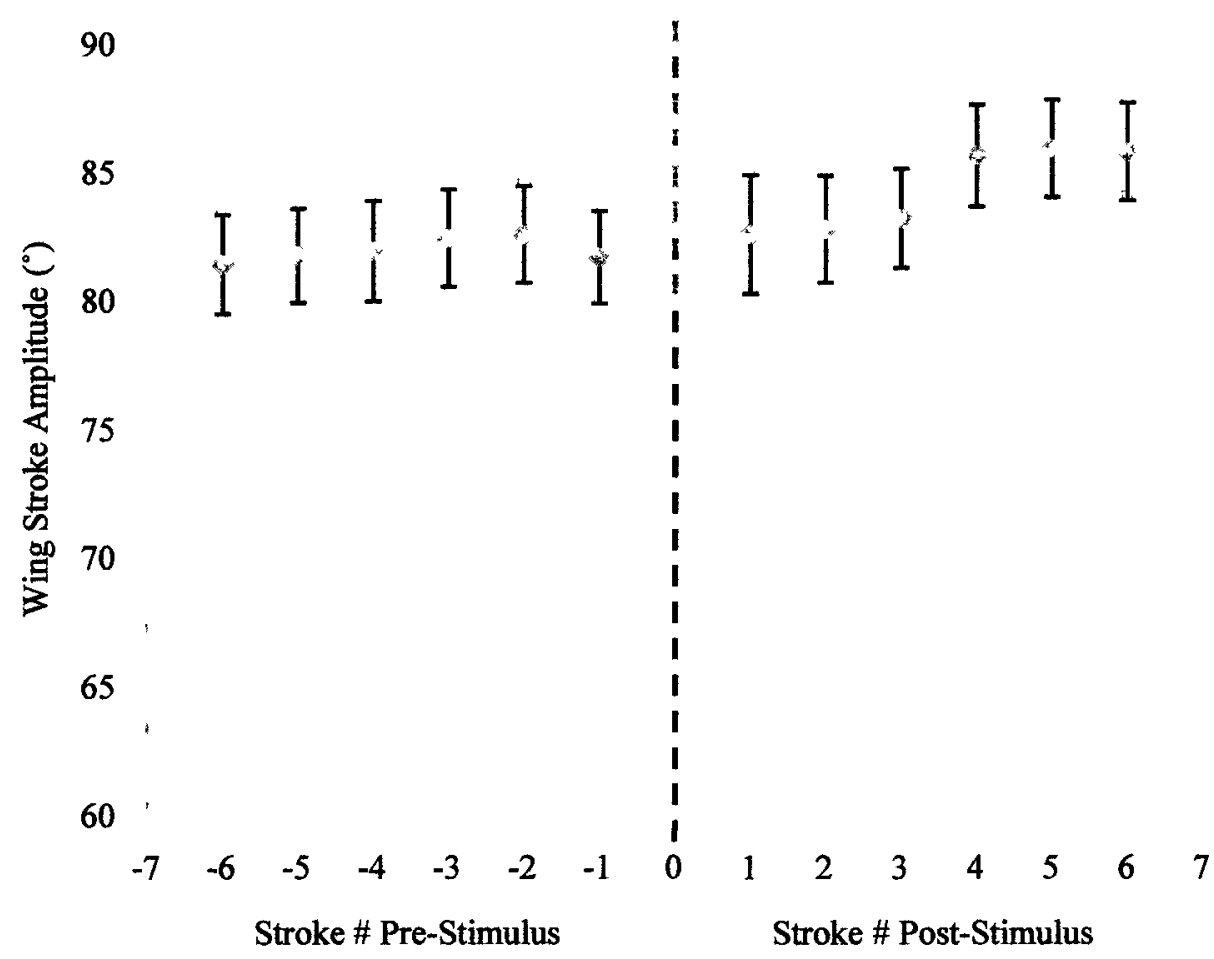

Figure 2.13. Wing stroke amplitudes of the ipsilateral hindwings of all locusts (A) and the average (B). Dashed red line indicates when the acoustic stimulus was introduced. There were no significant differences from pre- to post-stimulus. Note locust 01/11-4 (A) appears to be an outlier resulting in a significant difference between the ipsilateral and contralateral hindwings pre-stimulus. When this trial is removed, no significant difference occurs indicating this is a spurious result. Standard error is represented by error bars. 
A

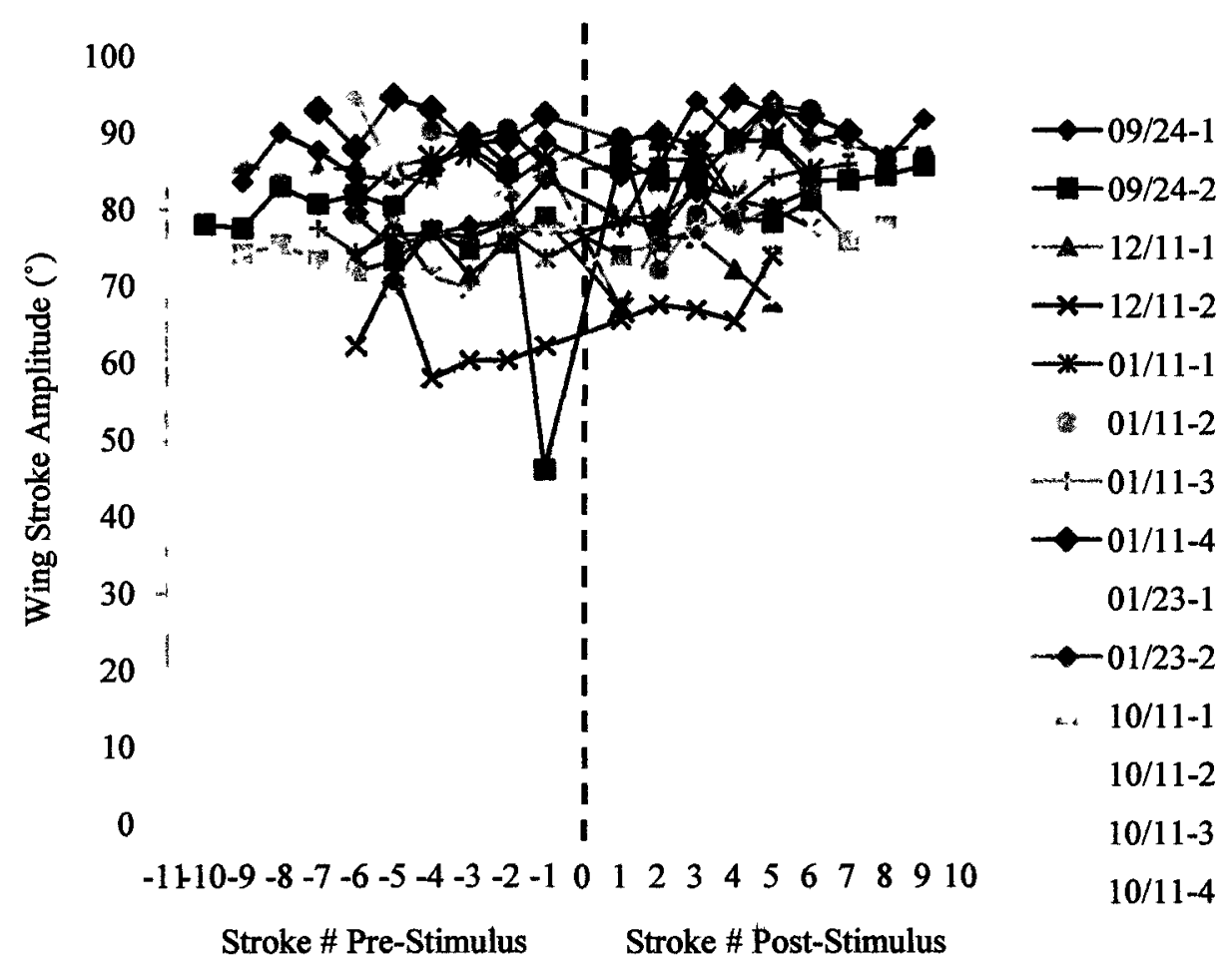

B

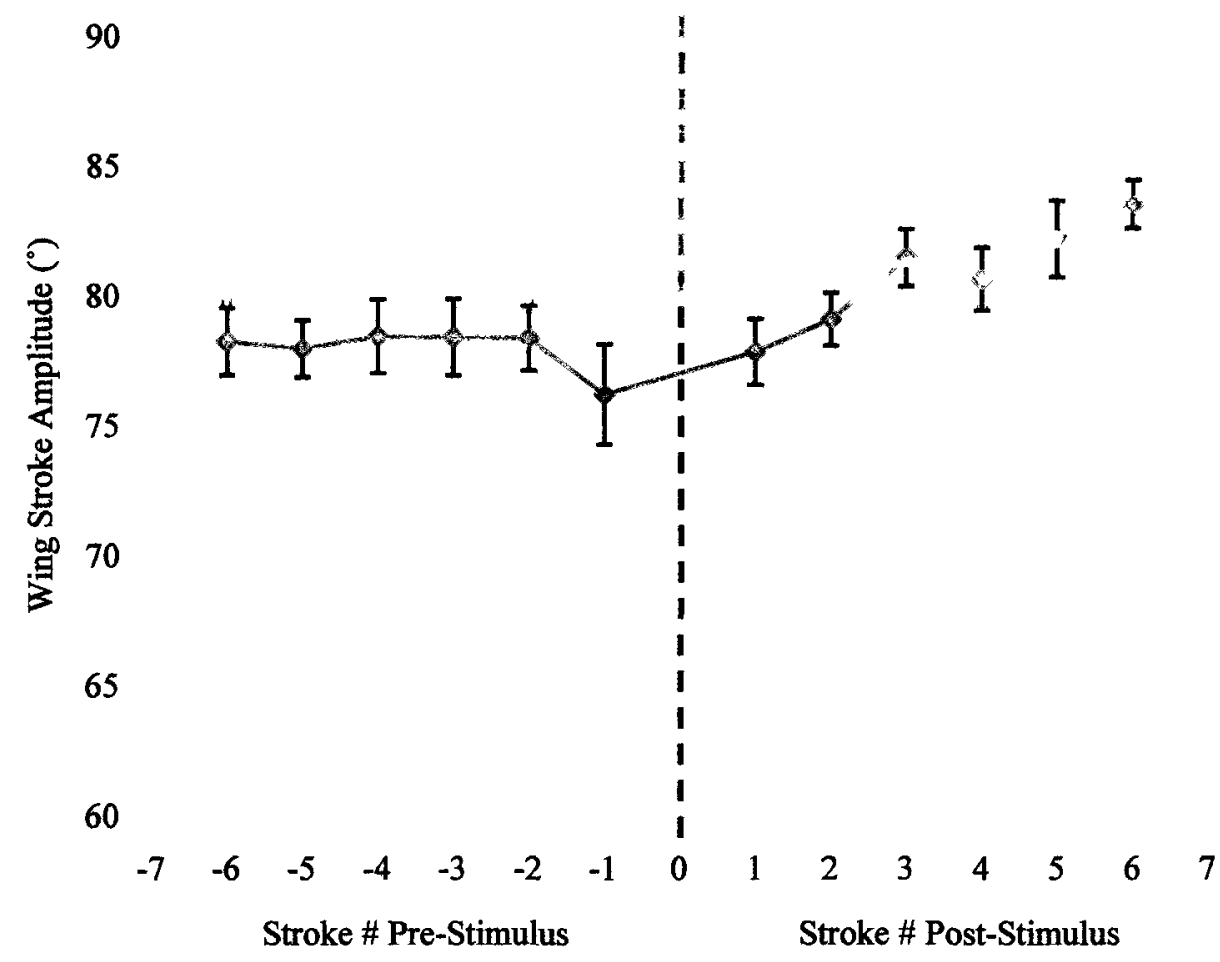

Figure 2.14. Wing stroke amplitudes of the contralateral hindwings of all locusts (A) and the average (B). Dashed red line indicates when the acoustic stimulus was introduced. There were no significant differences from pre- to post-stimulus. Standard error is represented by error bars. 


\subsubsection{Forewing Asymmetry}

Forewing asymmetry, measured at $1 / 3^{\text {rd }}$ the downstroke, was measured for all wing stroke cycles for all locusts tested. For each locust, the pre-stimulus forewing asymmetry was normalized to a mean pre-stimulus of 0 degrees before post-stimulus magnitudes were compared. Of the total number of trials $(\mathrm{N}=14), 9$ locusts exhibited a presumed left turn direction and 5 locusts exhibited a presumed right turn direction (Table 2.2). In both cases of turn direction, there were significant differences in the changes in forewing asymmetry after the stimulus was applied: left $(t=3.479, n=9$, $\mathrm{p}=0.008)$ with an average of $3.56^{\circ} \pm 1.02^{\circ}\left(\max : 9.18^{\circ}, \mathrm{min}: 0.05^{\circ}\right)$, and right $(\mathrm{t}=6.458$, $\mathrm{n}=5, \mathrm{p}=0.003)$ with an average of $2.27^{\circ} \pm 0.35^{\circ}\left(\max : 3.06^{\circ}\right.$, $\left.\min : 1.28^{\circ}\right)$ (Figures 2.15 and 2.16).

\subsubsection{Separation Angle}

When comparing the wing couple on the inside of the turn path, there was no significant difference in separation angle after a stimulus was applied $(t=-0.303, n=14$, $p=0.7670)$ with an average of $15.99^{\circ} \pm 3.58^{\circ}\left(\max : 22.98^{\circ}, \min : 2.77^{\circ}\right)($ Figure 2.17$)$. The wing couple on the outside of the turn path also showed no significant difference after the stimulus was applied $(\mathrm{t}=-0.617, \mathrm{n}=14, \mathrm{p}=0.5478)$ with an average of $15.62^{\circ} \pm$ $4.75^{\circ}\left(\max : 20.88^{\circ}, \min : 2.77^{\circ}\right)($ Figure 2.18$)$.

I also evaluated whether there were any significant differences in separation angle between the ipsilateral and contralateral wing couples pre-stimulus and post- 
A

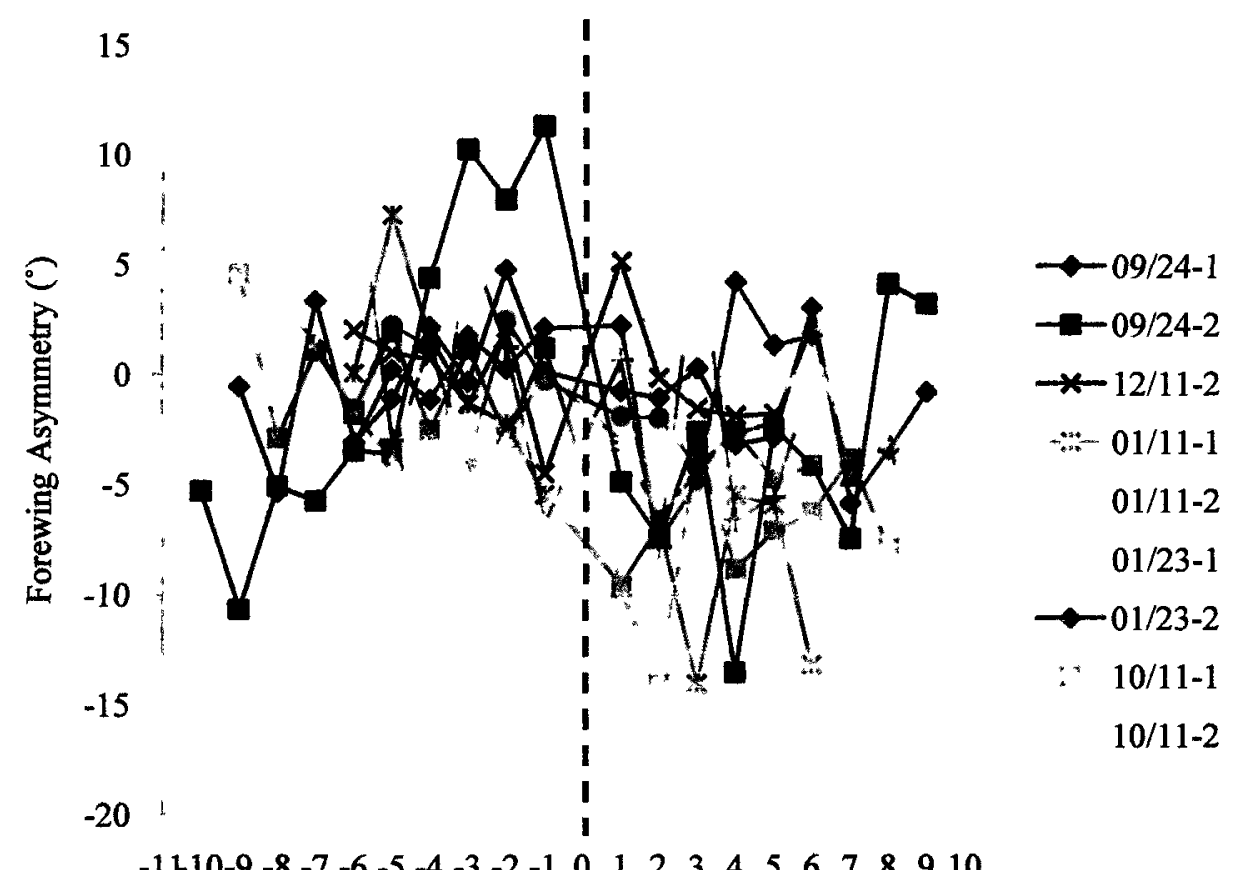

Stroke \# Pre-Stimulus Stroke \# Post-Stimulus

B

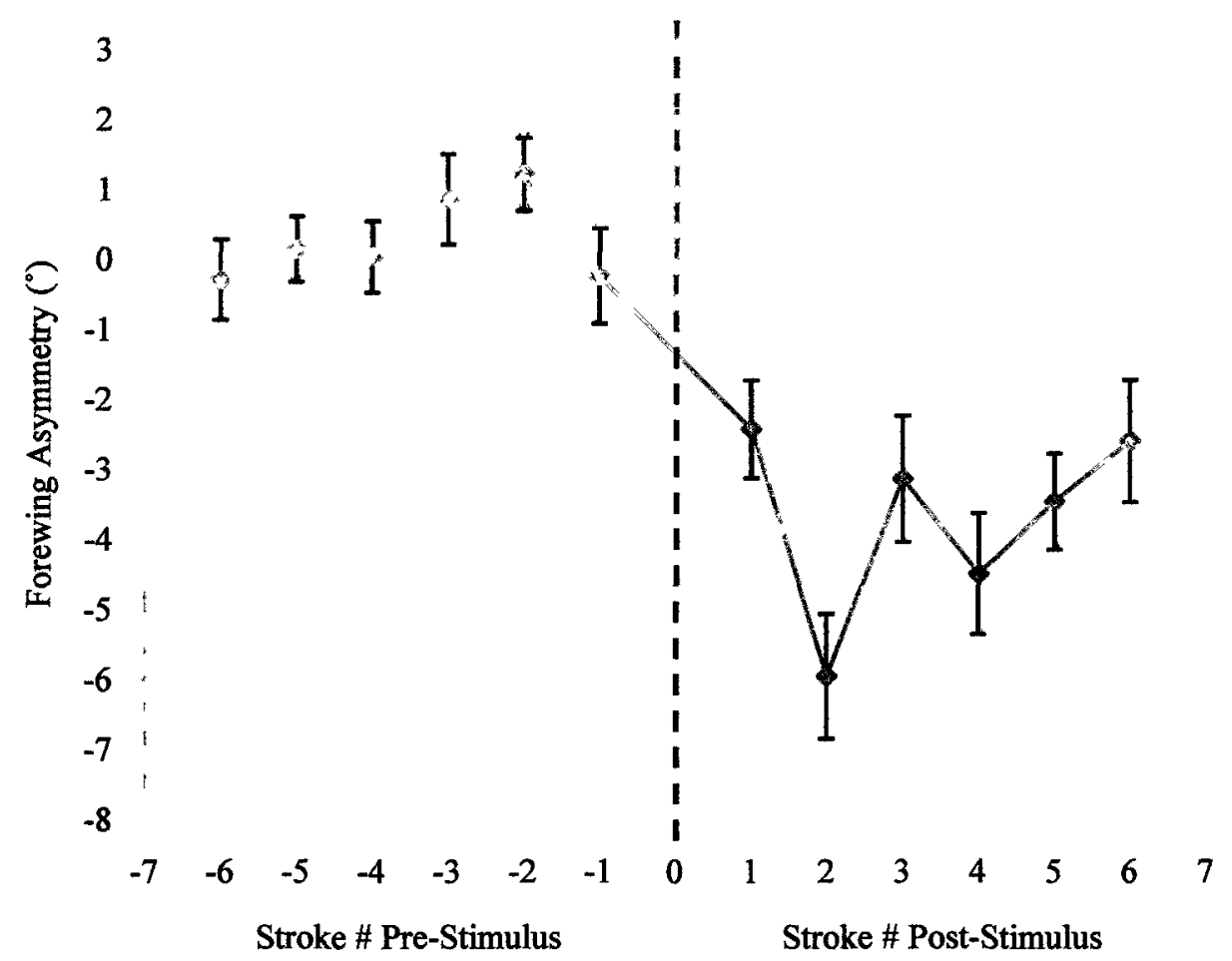

Figure 2.15. Forewing asymmetry of all left turning locusts (A) and the average (B). The dashed red line indicates when the acoustic stimulus was introduced. There was a significant difference in forewing asymmetry from pre- to post-stimulus $(t=3.479$, $n=9, p=0.008$ ). Note negative values indicate a left turn. Standard error is represented by error bars. 
A

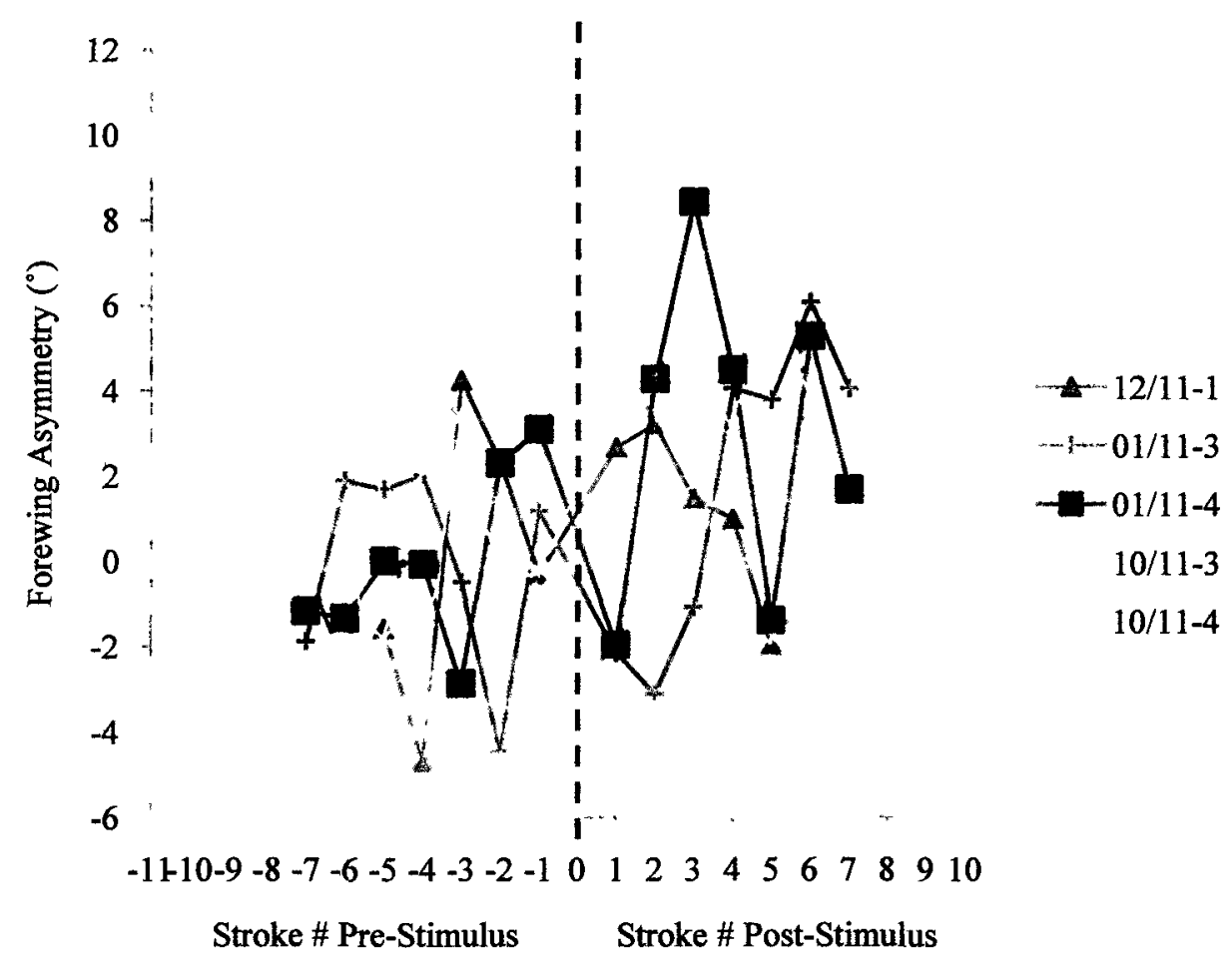

B

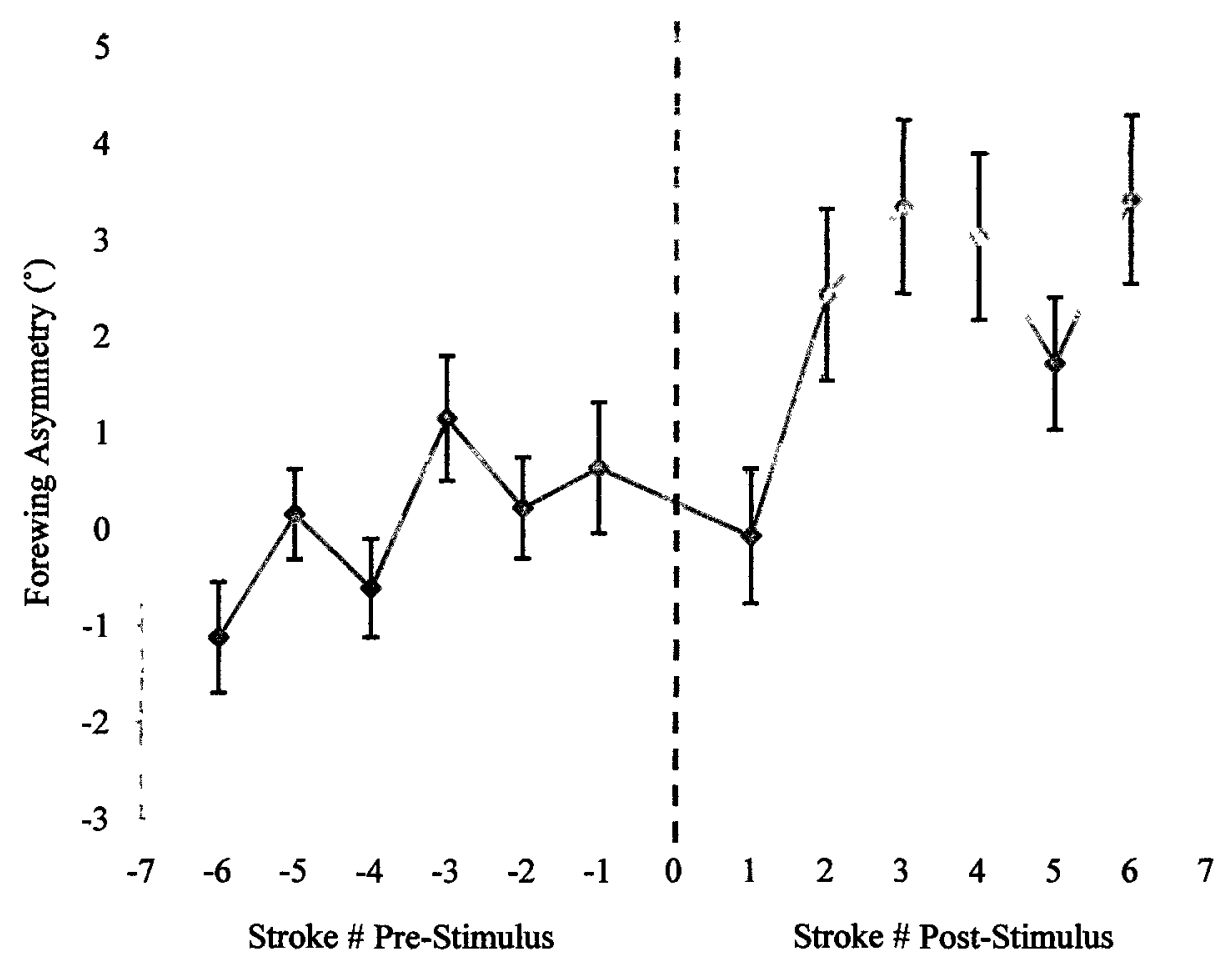

Figure 2.16. Forewing asymmetry of all right turning locusts (A) and the average (B). The dashed red line indicates when the acoustic stimulus was introduced. There was a significant difference in forewing asymmetry from pre- to post-stimulus $(t=6.458$, $n=5, p=0.003$ ). Note positive values indicate a right turn. Standard error is represented by error bars. 
A

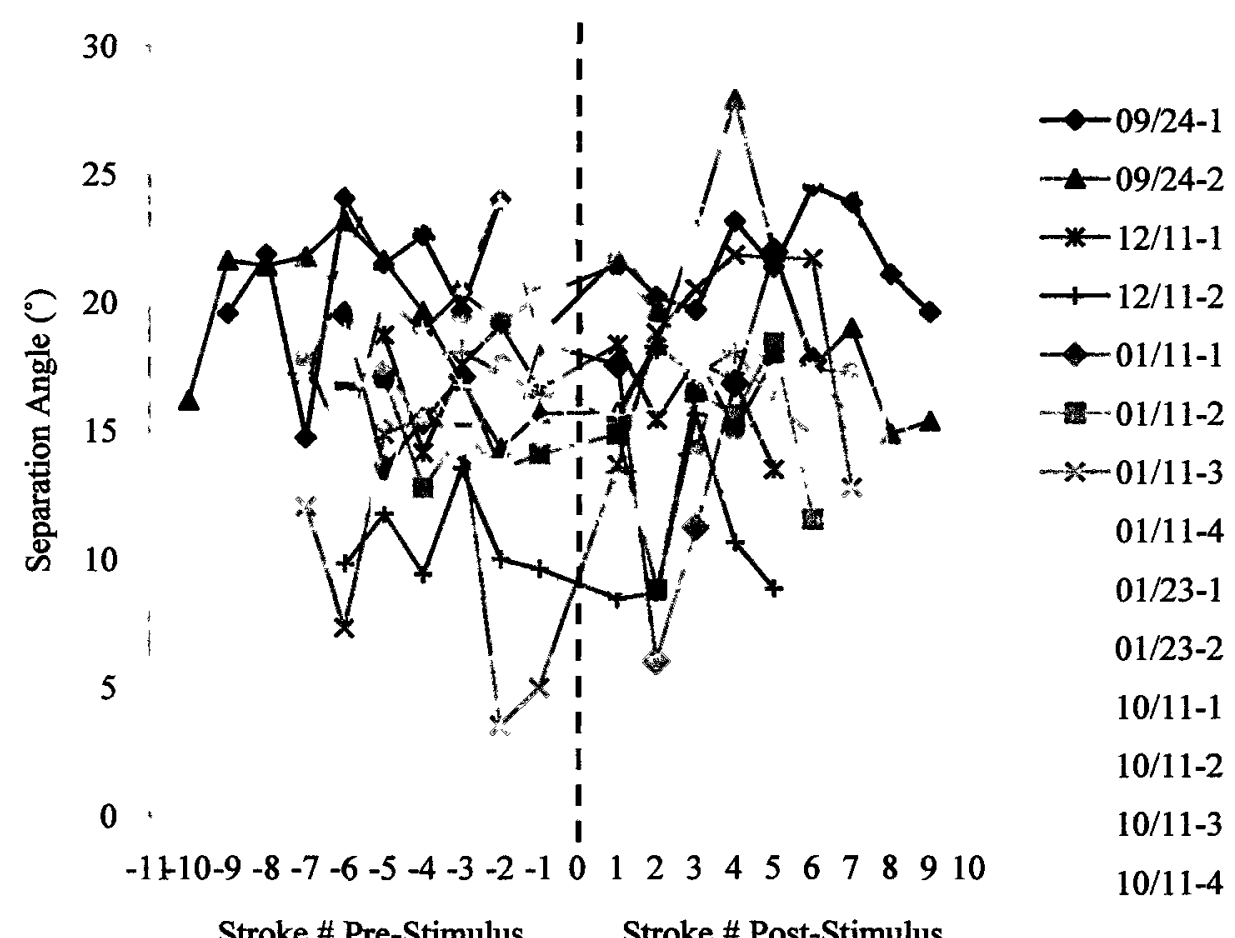

B

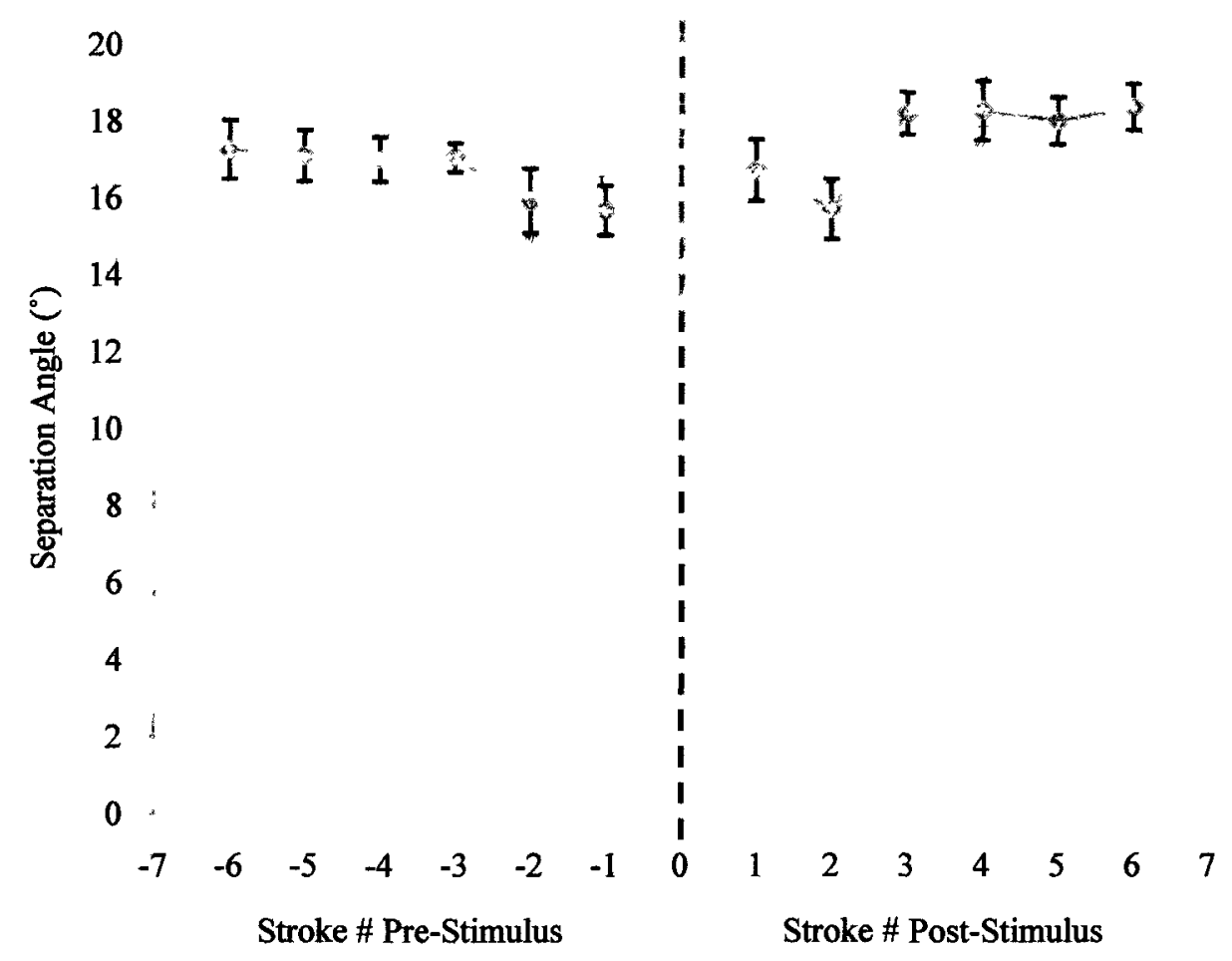

Figure 2.17. Separation angles of the ipsilateral wing pairs of all locusts (A) and the average (B). Dashed red line indicates when the acoustic stimulus was introduced. There was no significant difference in separation angles after the stimulus was applied. Standard error is represented by error bars. 
A

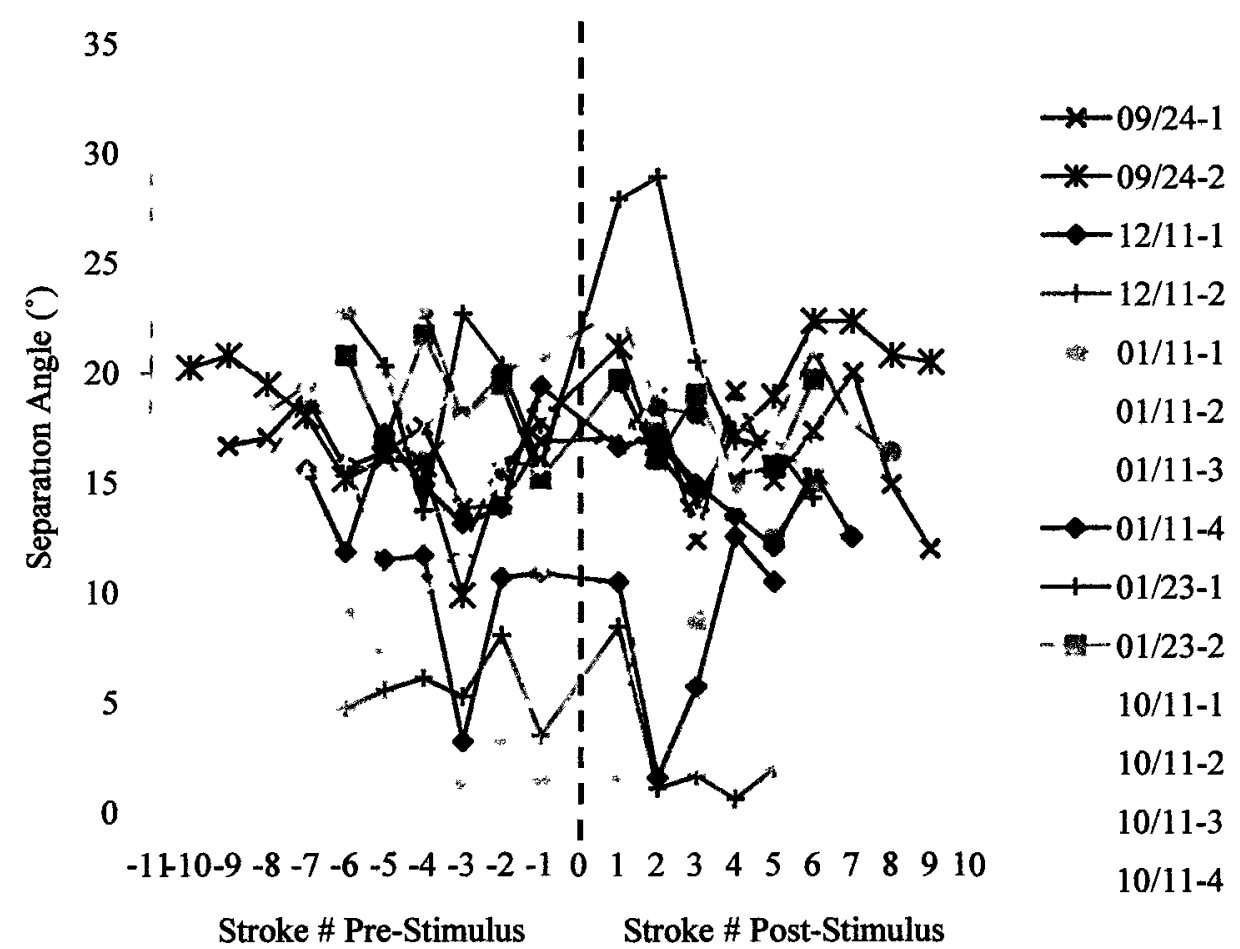

B

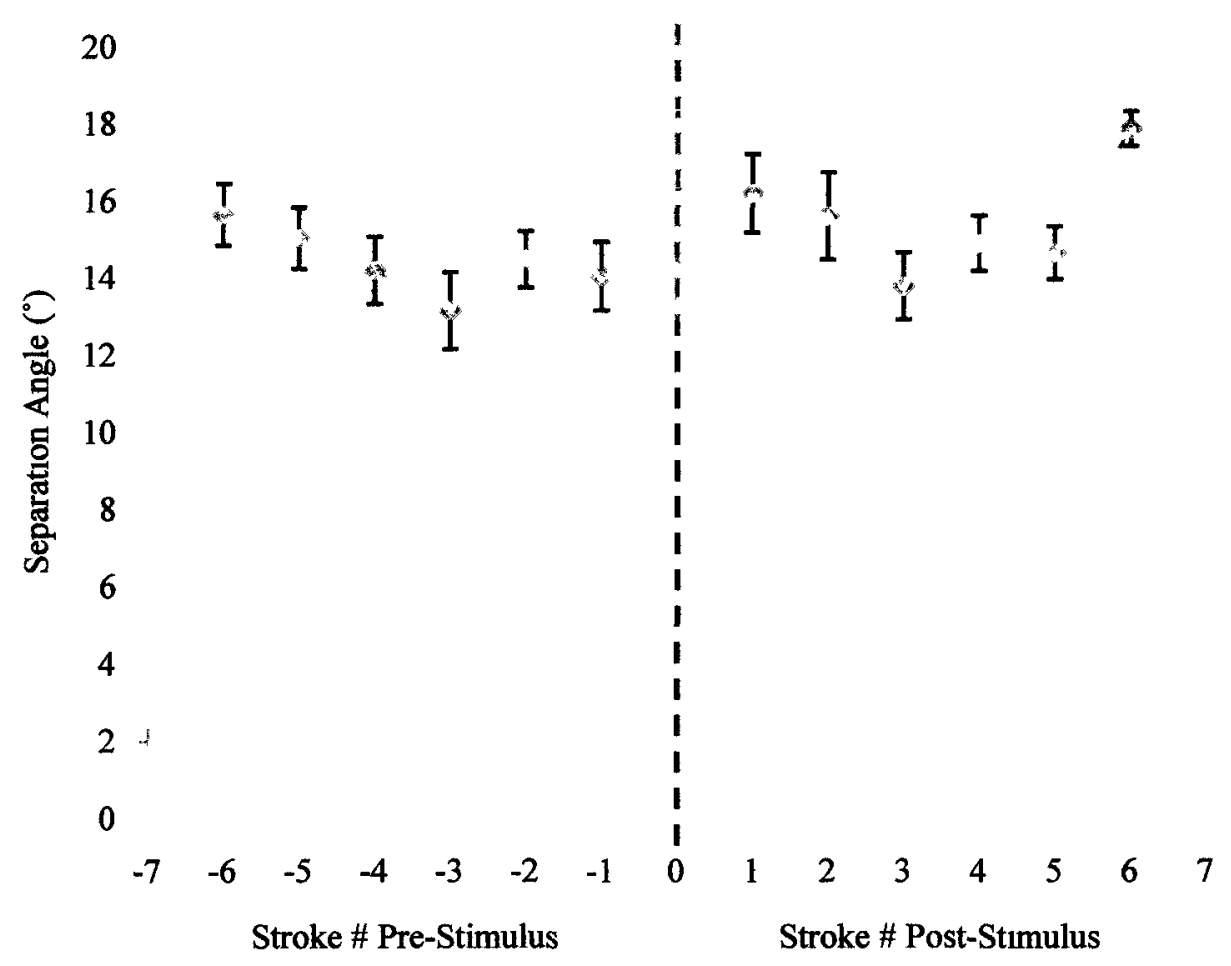

Figure 2.18. Separation angles of the contralateral wing pairs of all locusts (A) and the average (B). Dashed red line indicates when the acoustic stimulus was introduced. There was no significant difference in separation angles after the stimulus was applied. Standard error is represented by error bars. 
stimulus. Neither case showed any significant differences, meaning one side of the locust did not exhibit a significantly larger separation angle than the other. Pre-stimulus, the results showed $(t=0.456, n=14, p=0.6560)$, ipsi with an average of $15.92^{\circ} \pm 3.59^{\circ}$ (max: $\left.22.98^{\circ}, \min : 8.16^{\circ}\right)$, and contra with an average of $15.31^{\circ} \pm 5.03^{\circ}\left(\max : 20.58^{\circ}, \min :\right.$ $\left.4.07^{\circ}\right)$. Post-stimulus, the $t$-test showed a result of $(t=0.120, n=14, p=0.3976)$, ipsi with an average of $16.06^{\circ} \pm 3.56^{\circ}\left(\max : 21.67^{\circ}\right.$, min: $\left.8.16^{\circ}\right)$, and contra with an average of $15.92^{\circ} \pm 4.46^{\circ}\left(\max : 20.88^{\circ}, \min : 2.77^{\circ}\right)$. Separation angle data are summarized in Table 2.4 .

\subsubsection{Angular Difference}

A significant difference between the angular difference on either side of the locust (ipsilateral and contralateral wings) would result in asymmetric wing depression on one side (angular difference, $\mathrm{AD}$ ) and aid in steering maneuvers associated with the ASR of a locust; however; throughout the 14 trials, there were no significant differences between the ipsilateral and contralateral wings after the stimulus was applied. Pre-stimulus, the ttest showed a non-significant result $(\mathrm{t}=-0.212, \mathrm{n}=14, \mathrm{p}=0.8356)$, ipsi with an average of $10.44^{\circ} \pm 10.47^{\circ}\left(\max : 30.50^{\circ}\right.$, min: $\left.-5.60^{\circ}\right)$, and contra with an average of $11.64^{\circ} \pm$ $14.74^{\circ}$ (max: $33.74^{\circ}$, min: $-15.20^{\circ}$ ). Post-stimulus, the t-test showed a non-significant result $(\mathrm{t}=-0.182, \mathrm{n}=14, \mathrm{p}=0.8583)$, ipsi with an average of $11.52^{\circ} \pm 8.99^{\circ}\left(\max : 28.82^{\circ}\right.$, min: $\left.-4.31^{\circ}\right)$, and contra with an average of $12.38^{\circ} \pm 12.53^{\circ}\left(\max : 32.18^{\circ}\right.$, $\left.\min :-12.43^{\circ}\right)$.

When looking at the ipsilateral and contralateral wings independently from one another to see if changes in $\mathrm{AD}$ occurred after a stimulus was introduced, again, the 
Table 2.4. Summary of separation angles and angular differences measured for locust trials in the wind tunnel. Results are measured at $1 / 3^{\text {rd }}$ the downstroke and are averaged over 5-10 wing strokes pre-stimulus and 5-10 wing strokes post-stimulus.

\begin{tabular}{|c|c|c|c|c|c|c|c|c|c|c|c|}
\hline \multirow[b]{2}{*}{ Locust } & \multirow[b]{2}{*}{$\begin{array}{l}\text { Wing } \\
\text { Pair }\end{array}$} & \multicolumn{5}{|c|}{ Separation Angle $(\mathrm{SA})\left(^{\circ}\right)$} & \multicolumn{5}{|c|}{ Angular Difference (AD) $\left(^{\circ}\right)$} \\
\hline & & Mean & $\begin{array}{l}\text { MAX Pre- } \\
\text { Stimulus }\end{array}$ & $\begin{array}{l}\text { MAX Post- } \\
\text { Stimulus }\end{array}$ & $\begin{array}{l}\text { MIN Pre- } \\
\text { Stimulus }\end{array}$ & $\begin{array}{l}\text { MIN Post- } \\
\text { Stimulus }\end{array}$ & Mean & $\begin{array}{l}\text { MAX Pre- } \\
\text { Stimulus }\end{array}$ & $\begin{array}{l}\text { MAX Post- } \\
\text { Stimulus }\end{array}$ & $\begin{array}{l}\text { MN Pre- } \\
\text { Stimulus }\end{array}$ & $\begin{array}{l}\text { MNN Post- } \\
\text { Stimulus }\end{array}$ \\
\hline $09 / 24-1$ & \multirow{14}{*}{ Left } & 21.20 & 24.05 & 24.50 & 14.71 & 19.62 & 25.52 & 29.58 & 30.19 & 21.01 & 19.66 \\
\hline $09 / 24-2$ & & 19.24 & 23.16 & 27.88 & 14.31 & 14.84 & 29.70 & 36.43 & 33.06 & 27.55 & 24.18 \\
\hline $12 / 11-1$ & & 16.80 & 19.08 & 18.34 & 14.15 & 13.47 & 1.18 & 3.66 & 6.56 & -7.88 & 1.43 \\
\hline $12 / 11-2$ & & 10.58 & 13.54 & 15.60 & 9.42 & 8.45 & 13.80 & 15.77 & 26.82 & 5.41 & 4.58 \\
\hline $01 / 11-1$ & & 15.73 & 19.51 & 21.94 & 13.55 & 6.02 & 13.20 & 18.05 & 31.79 & 9.27 & 2.36 \\
\hline $01 / 11-2$ & & 14.54 & 17.06 & 18.40 & 12.78 & 8.79 & 1.97 & 3.33 & 26.09 & -8.41 & -5.85 \\
\hline $01 / 11-3$ & & 14.72 & 15.52 & 21.83 & 3.52 & 12.77 & 27.36 & 32.22 & 37.76 & 21.30 & 23.08 \\
\hline $01 / 11-4$ & & 18.38 & 20.38 & 21.42 & 14.32 & 15.02 & -9.80 & 13.32 & -5.08 & -24.32 & -16.84 \\
\hline $01 / 23-1$ & & 16.48 & 18.07 & 18.24 & 12.11 & 14.74 & -2.17 & 4.56 & 9.42 & -11.96 & -2.77 \\
\hline $01 / 23-2$ & & 16.31 & 19.43 & 19.24 & 14.65 & 13.18 & 8.16 & 14.99 & 14.54 & 2.23 & 5.04 \\
\hline $10 / 11-1$ & & 22.95 & 27.81 & 26.47 & 18.84 & 13.56 & -1.16 & 5.13 & 0.00 & -0.98 & -11.29 \\
\hline $10 / 11-2$ & & 16.80 & 20.82 & 22.20 & 14.01 & 10.26 & 13.86 & 19.87 & 16.26 & 11.25 & 7.97 \\
\hline $10 / 11-3$ & & 17.98 & 19.64 & 23.74 & 16.06 & 15.58 & 13.41 & 14.30 & 16.81 & 10.70 & 11.29 \\
\hline $10 / 11-4$ & & 14.90 & 18.87 & 17.91 & 12.58 & 9.15 & 19.21 & 24.19 & 26.64 & 14.23 & 14.42 \\
\hline $09 / 24-1$ & \multirow{8}{*}{ Right } & 16.33 & 18.94 & 20.03 & 13.86 & 11.99 & 4.37 & 8.62 & 9.39 & 0.52 & 0.51 \\
\hline $09 / 24-2$ & & 18.03 & 20.79 & 22.34 & 9.87 & 14.24 & -13.89 & -7.63 & -6.42 & -27.84 & -19.10 \\
\hline $12 / 11-1$ & & 8.88 & 11.69 & 12.55 & 3.22 & 1.58 & -1.51 & 2.62 & 6.79 & -8.96 & -3.54 \\
\hline $12 / 11-2$ & & 4.28 & 8.05 & 8.46 & 3.50 & 0.61 & 33.03 & 35.27 & 35.80 & 32.03 & 27.64 \\
\hline $01 / 11-1$ & & 7.86 & 9.14 & 17.31 & 1.36 & 1.77 & 11.21 & 15.71 & 17.25 & 6.24 & 5.76 \\
\hline $01 / 11-2$ & & 16.11 & 18.33 & 18.38 & 14.77 & 11.21 & 15.46 & 19.70 & 22.99 & 12.55 & 11.27 \\
\hline $01 / 11-3$ & & 15.33 & 14.96 & 19.66 & 12.80 & 13.14 & 14.11 & 17.95 & 19.26 & 7.37 & 5.81 \\
\hline $01 / 11-4$ & & 14.88 & 19.36 & 17.19 & 11.84 & 12.08 & 8.60 & 11.90 & 14.01 & 1.03 & 7.32 \\
\hline
\end{tabular}




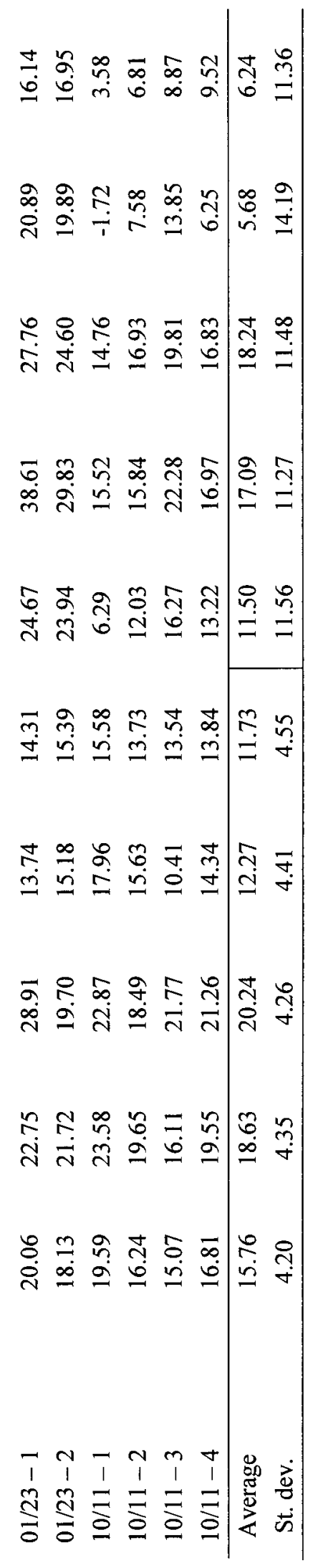


results show no significant differences, ipsi $(t=-1.089, n=14, p=0.2960)$ with an average of $10.98^{\circ} \pm 9.73^{\circ}\left(\max : 30.50^{\circ}\right.$, min: $\left.-5.60^{\circ}\right)$, and contra $(t=-0.829, n=14, p=0.4222)$ with an average of $12.01^{\circ} \pm 13.64^{\circ}\left(\max : 33.74^{\circ}\right.$, $\left.\min :-15.20^{\circ}\right)$ (Figures 2.19 and 2.20$)$

A summary of $\mathrm{AD}$ exhibited pre- and post-stimulus for both left and right wing pairs can be viewed in Table 2.4 .

\subsubsection{Dye-Flow Visualization}

\subsubsection{Kinematic Parameters}

The wing mounting device (chuck) allowed separation angle, angular difference and angle of attack of the forewing and hindwing to be adjusted independently to visualize any interactions between the wings that might be occurring. Goth (2010) showed that locusts flying in a wind tunnel produced forewing angles of attack during the downstroke that ranged between $20^{\circ}$ and $50^{\circ}$. The hindwing is a little more difficult to analyze due to the camber of the wing; however, it is said that the hindwing exhibits angles between $5^{\circ}-15^{\circ}$ (Jensen, 1956).

From the data collected for this study (above), I observed a range of separation angles between $0.61^{\circ}-28.91^{\circ}$ and angular differences between $-27.84^{\circ}-38.61^{\circ}$ (Table 2.4 ) These ranges of values are subsequently, for this thesis, considered to be 'biologically relevant' angles due to the fact that real locusts have been observed within those parameters. Any angles beyond the biologically relevant range are termed 'extreme' angles as a real locust would not typically exhibit those values during flight. 
A

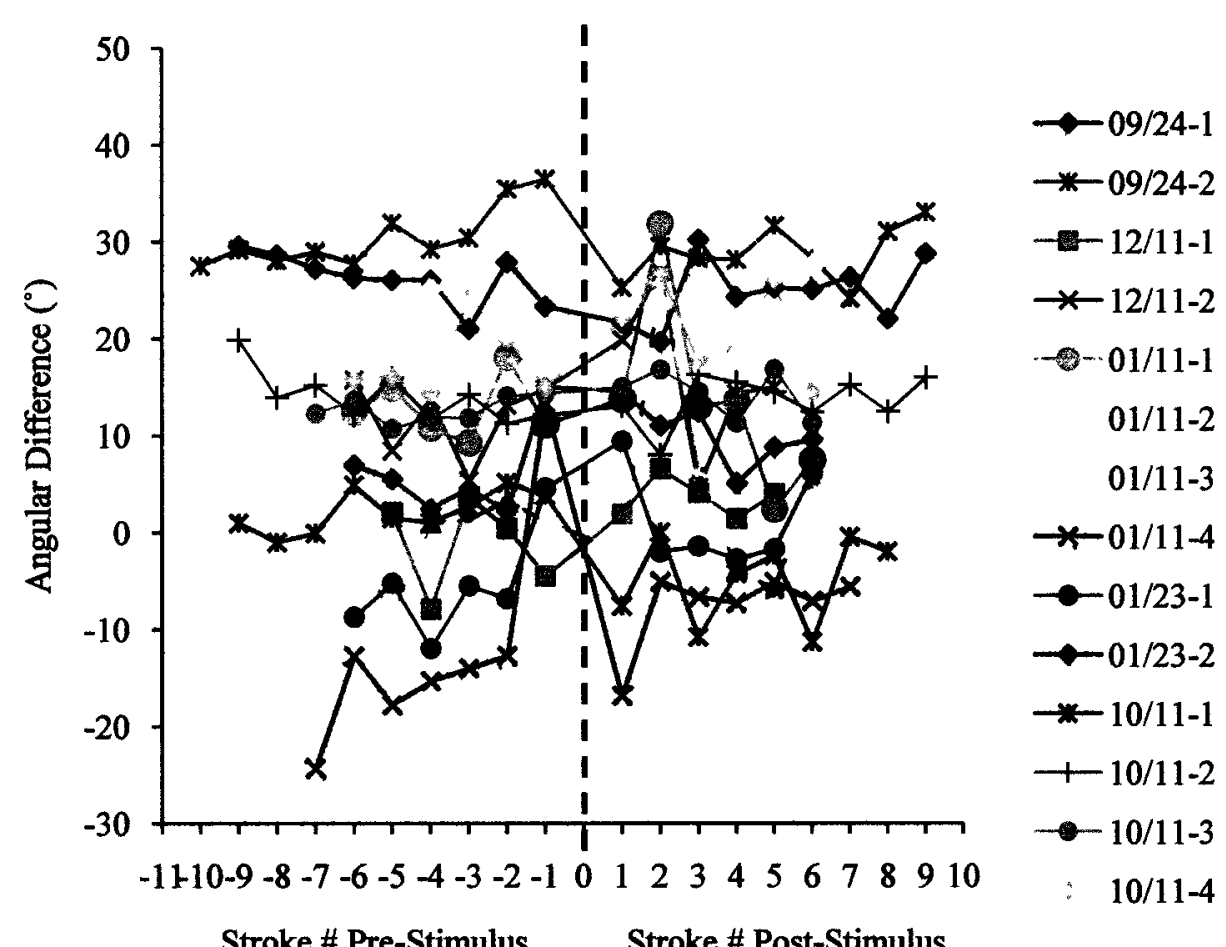

B

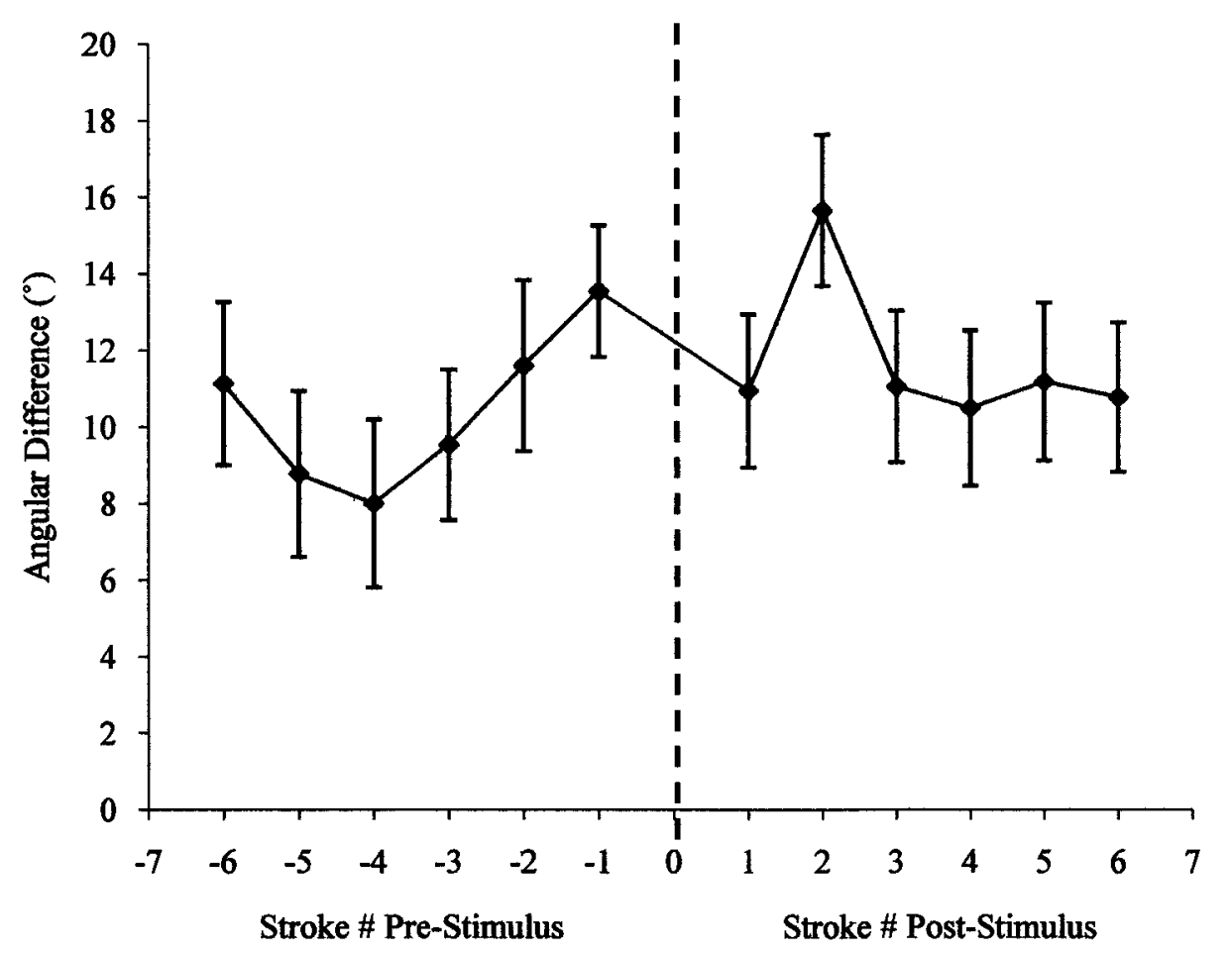

Figure 2.19. Angular differences of the ipsilateral wing pairs of all locusts $(A)$ and the average (B). Dashed red line indicates when the acoustic stimulus was introduced. There was no significant difference in separation angles after the stimulus was applied. Standard error is represented by error bars. 
A

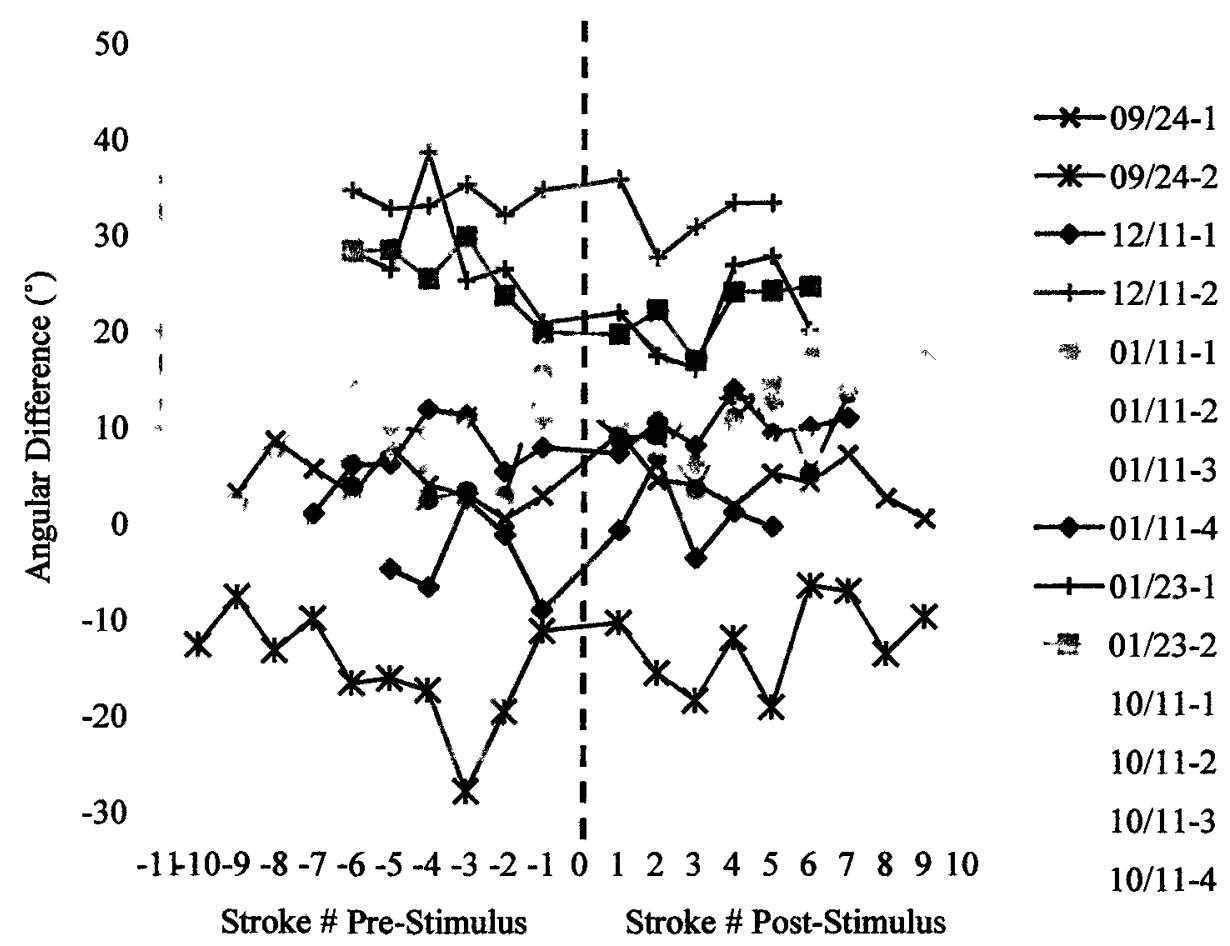

B

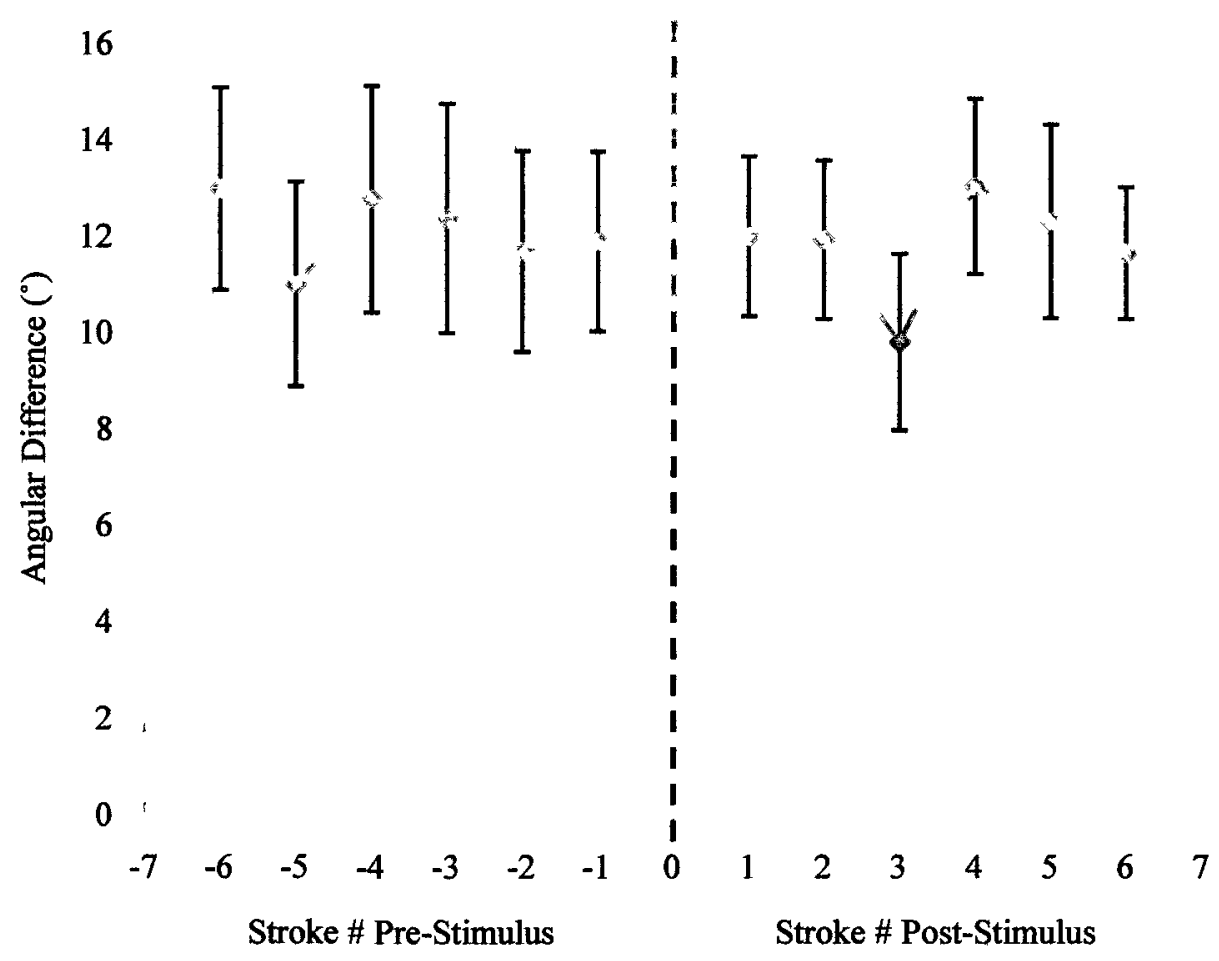

Figure 2.20. Angular differences of the contralateral wing pairs of all locusts (A) and the average (B). Dashed red line indicates when the acoustic stimulus was introduced. There was no significant difference in separation angles after the stimulus was applied. Standard error is represented by error bars. 
Both biologically relevant and extreme angles were used for the dye-flow visualization wing configurations to observe the effects on wing-wing interactions.

\subsubsection{Bulk Flow}

Dye-flow visualization allows for the observation of the gross structure of flow around a wing. In this case, when Malachite green crystals are placed at the base of a locust forewing or hindwing, depending on the angle of attack, the dye either flows off the surface of the wing or gets entrained into dual LEV structures (the minor LEV and primary LEV (using terminology of Lu et. al., 2006), which are then either shed or become entrained into the tip vortex.

When the angle of attack of the forewing was at an angle in which the vorticity of the LEV structure was too weak to entrain the dye $\left(\sim>10^{\circ}\right)$, the dye simply remained in the boundary layer where it travelled to the trailing edge and was shed. The dye from the forewing flowed over the bulk flow of the hindwing. In this case, the dye did not get entrained in to the tip vortex, in any turbulence associated with the trailing edge, nor did it get entrained in to the flows associated with the hindwing (Figure 2.21).

The hindwing exhibited similar results when one of three things occurred; 1) the hindwing was at an angle of attack too small to produce a LEV strong enough to entrain the dye at the base of the wing, 2) the interactions of flow from the forewing inhibited the hindwing from producing a visible separation bubble, or 3) a combination of the two factors. Typically a LEV structure will become visible at angles of attack greater than $10^{\circ}$. Sometimes LEV's would fail to either form or become visible on the hindwing due 

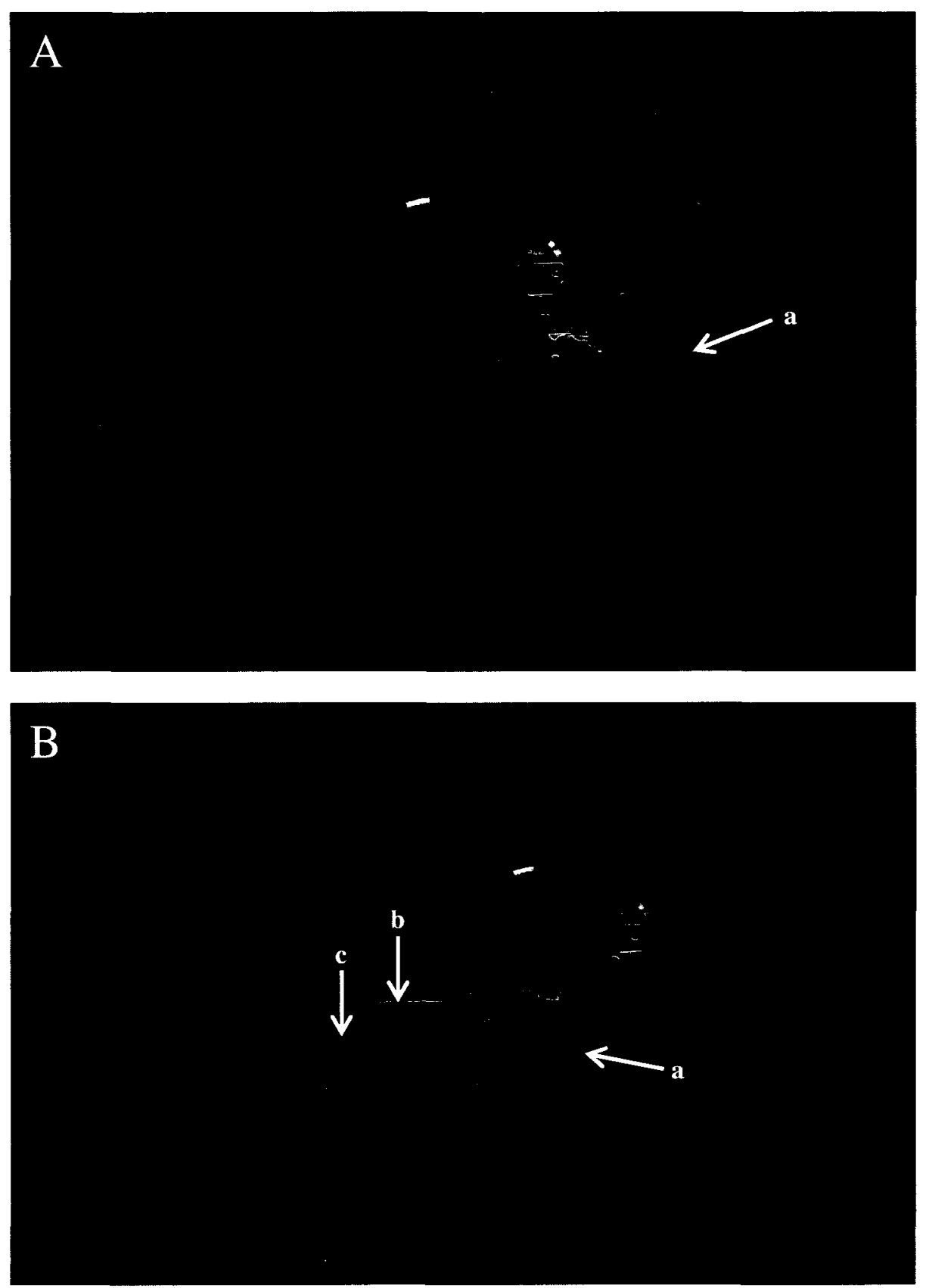

Figure 2.21. Photograph of a model locust wing pair in rotation. The wings are set to a configuration where the forewing is at an angle of attack of $5^{\circ}$, the hindwing at an angle of attack of $30^{\circ}$, an angular difference of $5^{\circ}$, a separation angle of $9^{\circ}$ and a rotation speed of $11.2 \mathrm{~s}$. (A) shows the dye from the forewing (a) flowing over the separation bubble of the hindwing. (B) shows minor (b) and primary (c) LEV structures forming on the hindwing and the flow from the forewing flowing over-top of those structures (a). 
to the interaction of the forewing until a combination of a higher hindwing angle of attack and a sufficiently small forewing angle of attack allowed for the development and visualization of the LEV structure. When LEV structures did not appear on the hindwing, other flow patterns and structures such as the tip vortex and shedding of the vorticies and the overall bulk flow could not be viewed because the dye was never entrained to extend to those areas to visualize them, it simply remained in the boundary layer on the surface of the wing until it was shed at the trailing edge (Figure 2.21).

Throughout the 85 trials conducted, observations show that dye shed from the forewing never becomes entrained with the flow structures on the hindwing, it simply flows over or around the bulk flow of the hindwing; therefore, observations suggest that the flow structures form independently on both the forewing and the hindwing and do not interact with one another; however, the flow structures on the forewing, depending on their magnitude as a result of different kinematic variables, can impede or inhibit the development of flow structures on the hindwing resulting in an overall effect of the forewing on the hindwing.

\subsubsection{Leading Edge Vortex}

Lu et. al. (2006), have shown that each vortex forms independently at the base of the wing and travels along the leading edge at different angles, where the smaller, minor LEV always running parallel to the leading edge of the wing at an angle of $0^{\circ}$, and the larger, primary LEV $\left(1^{\circ} \mathrm{LEV}\right)$ travelling at an angle relative to the angle of attack of the wing (Figure 2.22) 

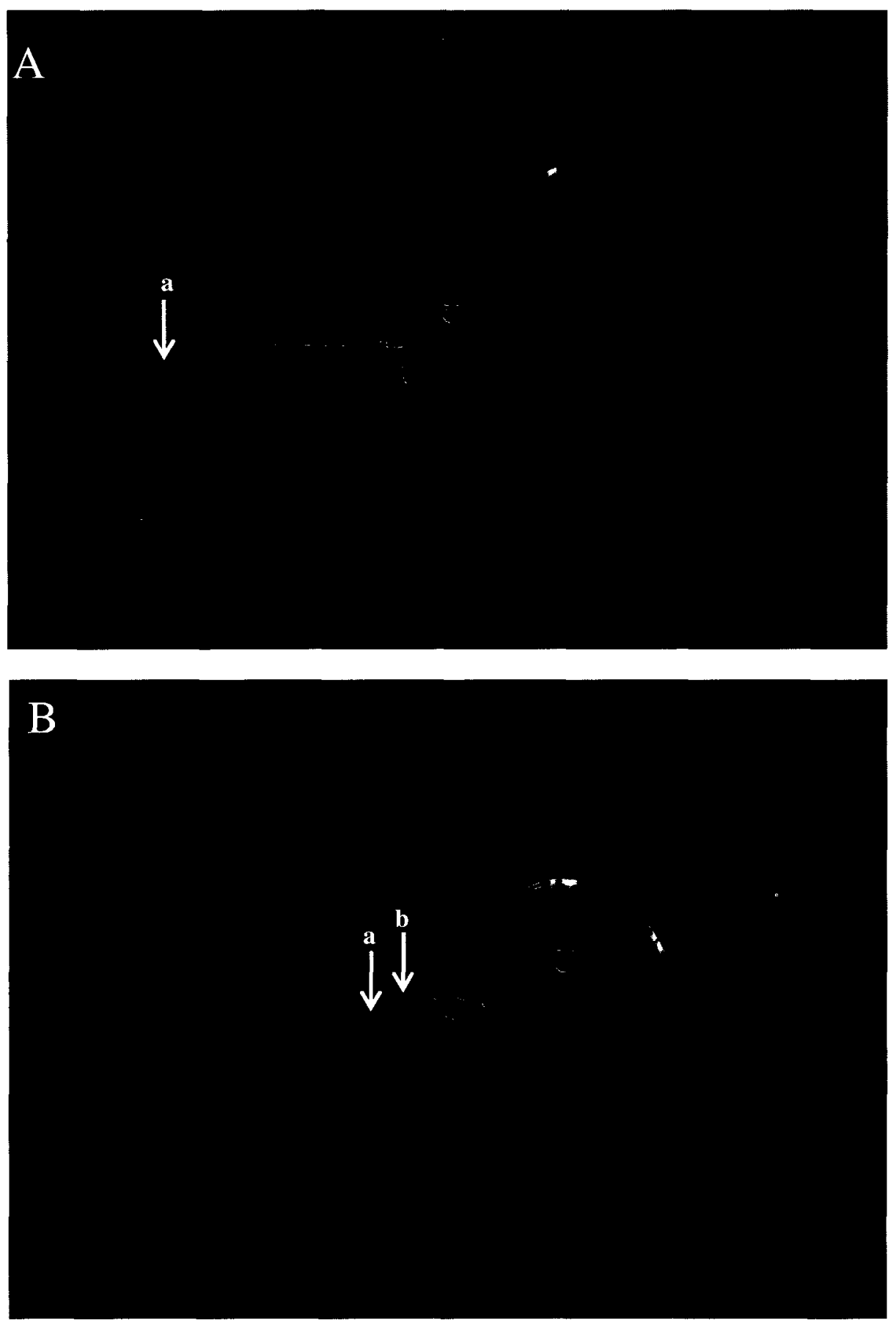

Figure 2.22. Photograph of a locust hindwing in rotation at an angle of attack of $15^{\circ}$ (A) and $30^{\circ}$ (B) at a rotation speed of $11.8 \mathrm{~s}$. (A) a primary LEV has formed along the leading edge of the wing (a). (B) a minor LEV runs parallel to the leading edge of the wing (b) and the primary LEV is running at an angle relative to the leading edge (a). 
LEV structures will begin to appear on both the fore- and hindwings at angles of attack of less than approximately $10^{\circ}$. The separation and visibility of both the minor and primary LEV structures, also termed dual LEV structures, appear at angles between $20^{\circ}$ and $25^{\circ}$ or greater (Table 2.5). This statement holds true for both the fore- and hindwings when spun in isolation in the spinner tank, and therefore there are no wing-wing interactions impeding the development of flow structures. When the wing pairs are spun together at various angles of attack, separation angles and angular differences, the development of LEV structures on the hindwing are affected but the LEV structures on the forewing appear to remain unchanged as there is no object in front of it to affect the flow. This is not to say that there are no wing-wing interactions occurring at all on the forewing as a result of the hindwing, but simply that the LEV structures do not appear to be affected as a result of the hindwing.

Observations of the LEV structures of the hindwing, as a result of the flow structures of the forewing, are as follows: 1) when the angle of attack was less than $5^{\circ}$, the hindwing was able to form a separation bubble (LEV) at angles greater than $15^{\circ}$ (Figure 2.23). 2) As the angle of attack of the forewing increases to angles greater than $5^{\circ}$, it appears to either impede or completely inhibit the formation of the LEV structures on the hindwing depending on the corresponding angle of the hindwing, meaning LEV structures are not visible on the hindwing at particular configurations are therefore assumed to be present but with a weak vorticity magnitude, or not present at all, until the hindwing reaches an angle of attack sufficient enough to produce LEV structures with a vorticity magnitude that are able to overcome the flow structures of the forewing, see table 2.6 for specific examples of wing configurations to produce this result (Figure 
Table 2.5. Results of dye-flow observations for a hindwing in isolation rotated in the spinner tank

\begin{tabular}{cccccc}
\hline HW $\alpha\left(^{\circ}\right)$ & $\begin{array}{c}\text { LEV on HW } \\
(\text { Yes or No) }\end{array}$ & $\begin{array}{c}\text { Dual LEV } \\
\text { Formation on HW } \\
\text { (Yes or No) }\end{array}$ & $\begin{array}{c}\text { Detachment Point } \\
(\mathrm{r} / \mathrm{R})\end{array}$ & Rotation Speed (s) & $\begin{array}{c}\text { Reynolds Number } \\
(\text { Re) of Hindwing }\end{array}$ \\
\hline 5 & No & No & N/A & 11.8 & 4300 \\
10 & No & No & N/A & 11.9 & 4300 \\
15 & Yes & No & 0.85 & 12.2 & 4300 \\
20 & Yes & No & 0.70 & 11.5 & 4300 \\
25 & Yes & Yes & 0.55 & 11.6 & 4300 \\
30 & Yes & Yes & 0.45 & 11.3 & 4300 \\
\hline
\end{tabular}


Table 2.6. Results of dye-flow observations for locust wing pair at varying angles of attack, separation angles and angular differences. Writing in Italic denotes replications of that particular wing configuration

\begin{tabular}{|c|c|c|c|c|c|c|c|c|c|c|}
\hline $\begin{array}{l}\text { SA } \\
\left({ }^{\circ}\right)\end{array}$ & $\begin{array}{l}\mathrm{AD} \\
\left({ }^{\circ}\right)\end{array}$ & $\begin{array}{c}\mathrm{FW} \\
\alpha \\
\left(^{\circ}\right)\end{array}$ & $\begin{array}{c}\mathrm{HW} \\
\alpha \\
\left(^{\circ}\right)\end{array}$ & $\begin{array}{l}\text { LEV on FW } \\
\text { (Yes or No) }\end{array}$ & $\begin{array}{c}\text { LEV on } \\
\text { HW } \\
\text { (Yes or No) }\end{array}$ & $\begin{array}{c}\text { Dual LEV } \\
\text { Formation on } \\
\text { FW } \\
\text { (Yes or } \mathrm{No})\end{array}$ & $\begin{array}{c}\text { Dual LEV } \\
\text { Formation on } \\
\text { HW } \\
\text { (Yes or No) }\end{array}$ & $\begin{array}{c}\text { Detachment } \\
\text { Point (r/R) }\end{array}$ & $\begin{array}{l}\text { Rotation } \\
\text { Speed } \\
\text { (s) }\end{array}$ & $\begin{array}{c}\text { Reynolds } \\
\text { Number } \\
\text { (Re) of } \\
\text { Hindwing }\end{array}$ \\
\hline 9 & 5 & 0 & 5 & No & No & N/A & N/A & N/A & 11.4 & 4300 \\
\hline 9 & 5 & 0 & 10 & No & Yes & N/A & No & 0.85 & 12.1 & 4300 \\
\hline 9 & 5 & 0 & 15 & No & Yes & N/A & No & 0.74 & 11.4 & 4300 \\
\hline 9 & 5 & 0 & 20 & No & Yes & N/A & Yes & 0.62 & 11.3 & 4300 \\
\hline 9 & 5 & 5 & 0 & No & No & $\mathrm{N} / \mathrm{A}$ & N/A & N/A & 12.1 & 4300 \\
\hline 9 & 5 & 5 & 5 & No & No & N/A & N/A & N/A & 12.2 & 4300 \\
\hline 9 & 5 & 5 & 10 & No & No & N/A & N/A & $\mathrm{N} / \mathrm{A}$ & 11.9 & 4300 \\
\hline 9 & 5 & 5 & 15 & No & Yes & $\mathrm{N} / \mathrm{A}$ & No & 0.92 & 11.7 & 4300 \\
\hline 9 & 5 & 5 & 15 & No & Yes & $N / A$ & No & 0.89 & 11.9 & 4300 \\
\hline 9 & 5 & 5 & 20 & No & Yes & N/A & Yes & 0.88 & 11.6 & 4300 \\
\hline 9 & 5 & 5 & 30 & No & Yes & N/A & Yes & 0.55 & 11.2 & 4300 \\
\hline 9 & 5 & 10 & 5 & Yes & No & No & N/A & N/A & 11.4 & 4300 \\
\hline 9 & 5 & 10 & 15 & Yes & No & No & N/A & N/A & 11.5 & 4300 \\
\hline 9 & 5 & 10 & 30 & Yes & Yes & No & Yes & 0.62 & 11.2 & 4300 \\
\hline 9 & 5 & 15 & 5 & Yes & No & No & $\mathrm{N} / \mathrm{A}$ & $\mathrm{N} / \mathrm{A}$ & 11.7 & 4300 \\
\hline 9 & 5 & 15 & 10 & Yes & No & No & N/A & N/A & 11.5 & 4300 \\
\hline 9 & 5 & 15 & 15 & Yes & No & No & $\mathrm{N} / \mathrm{A}$ & $\mathrm{N} / \mathrm{A}$ & 11.1 & 4300 \\
\hline 9 & 5 & 15 & 20 & Yes & Yes & No & No & 0.73 & 11.9 & 4300 \\
\hline 9 & 5 & 15 & 25 & Yes & Yes & No & No & 0.58 & 11.7 & 4300 \\
\hline 9 & 5 & 15 & 25 & Yes & Yes & No & No & 0.55 & 11.8 & 4300 \\
\hline 9 & 5 & 15 & 25 & Yes & Yes & No & No & 0.52 & 11.6 & 4300 \\
\hline
\end{tabular}




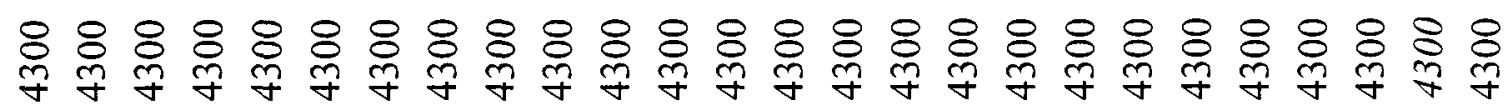

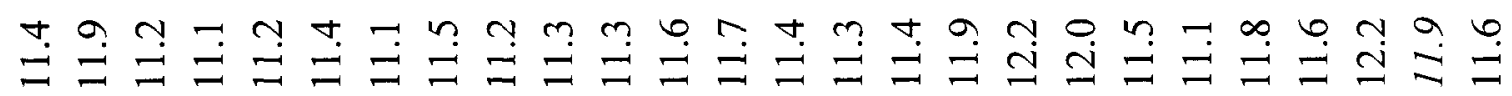

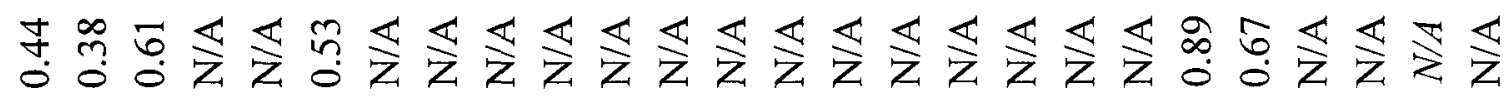

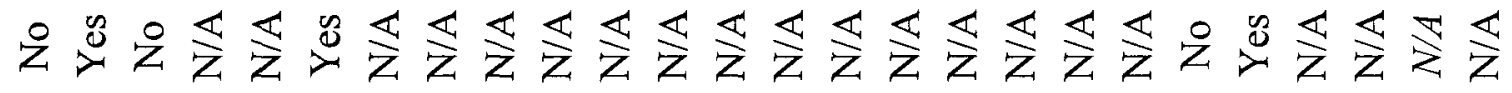

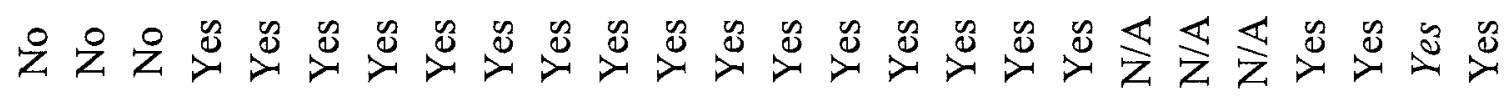

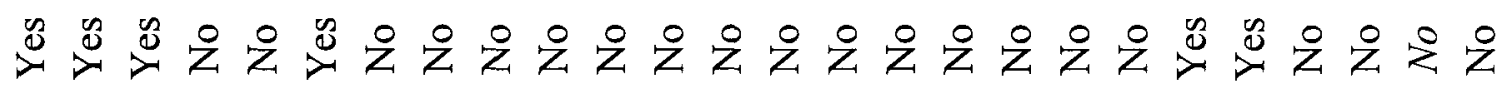

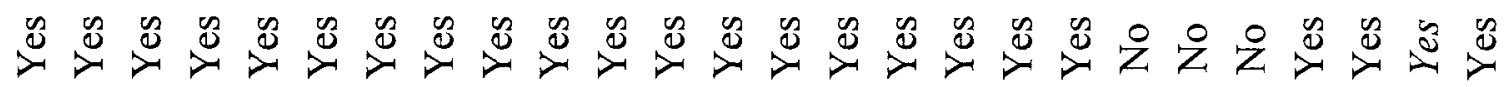

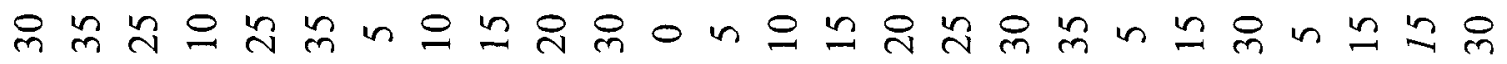

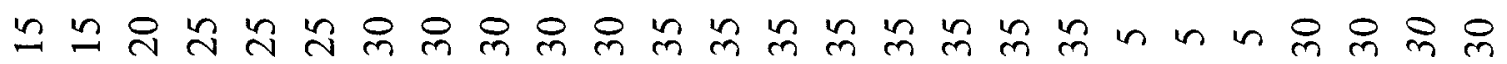

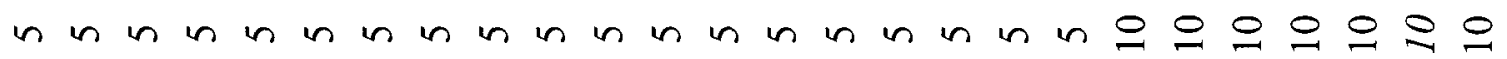

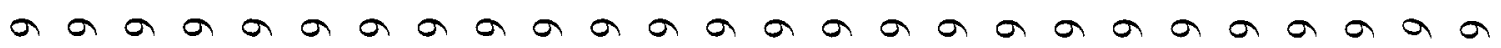




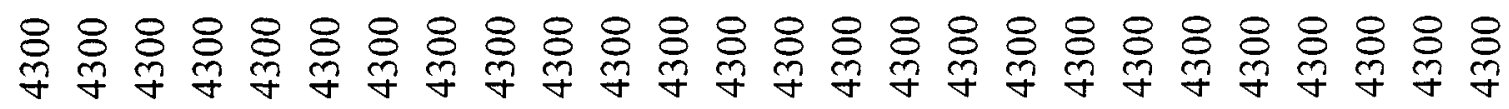

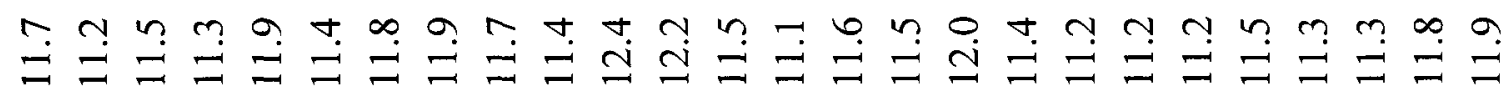

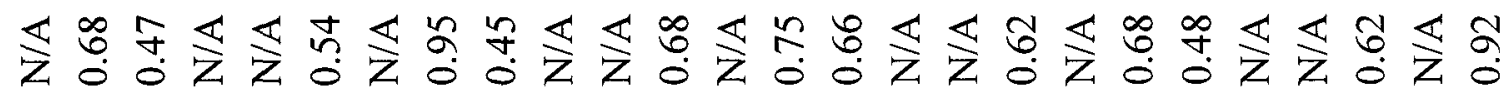

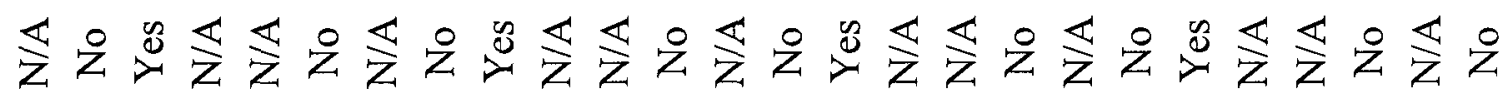

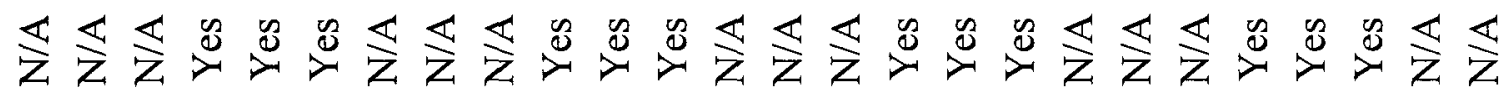

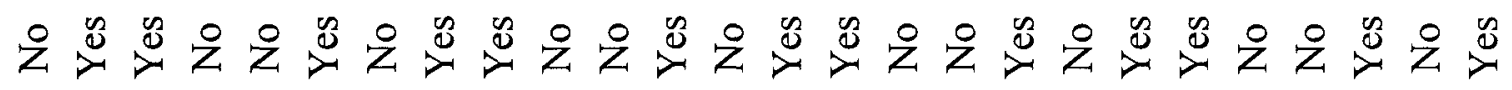

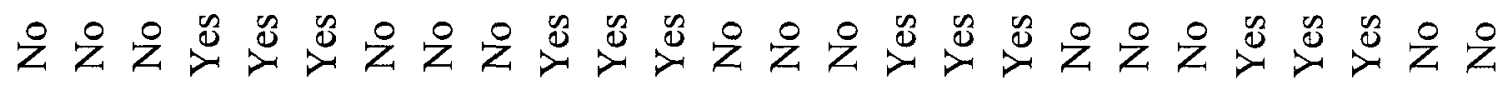

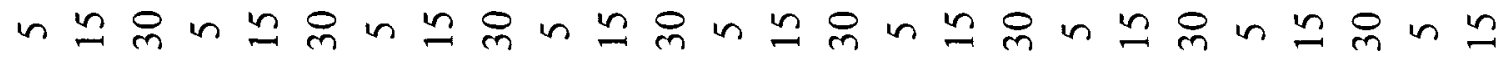

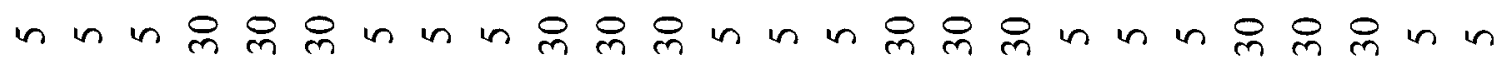

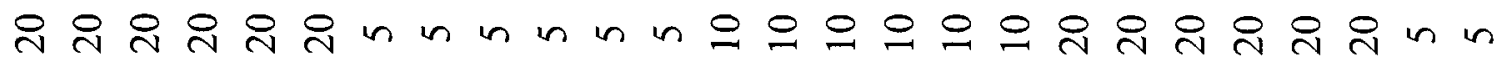

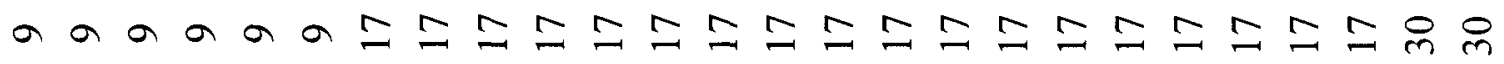




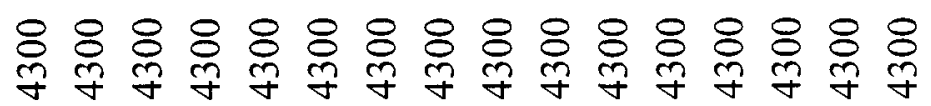
ઉั๋

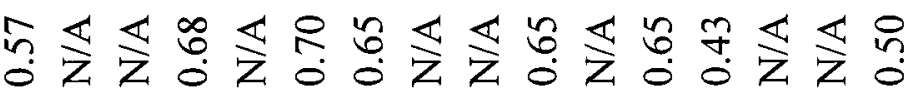

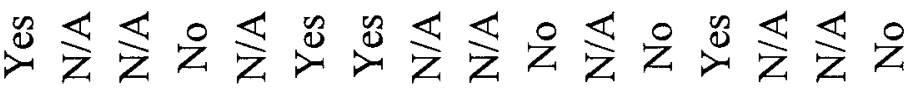

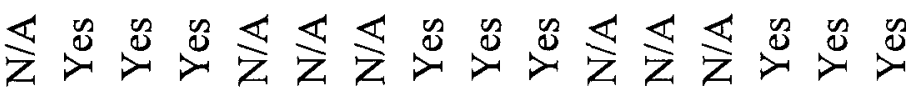

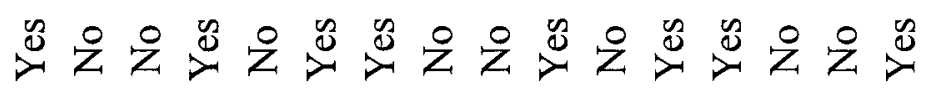

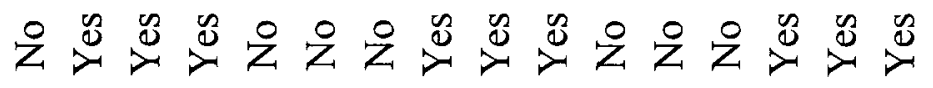

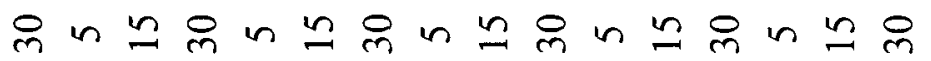

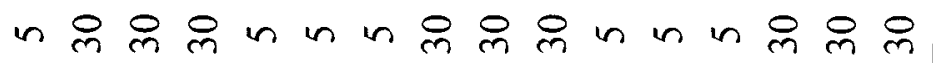
nn nn $n$ 으으으으으으윳ㅇㅅㅇ유유유

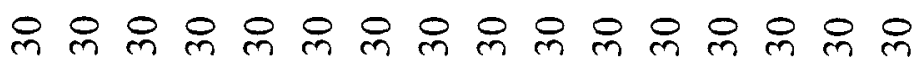



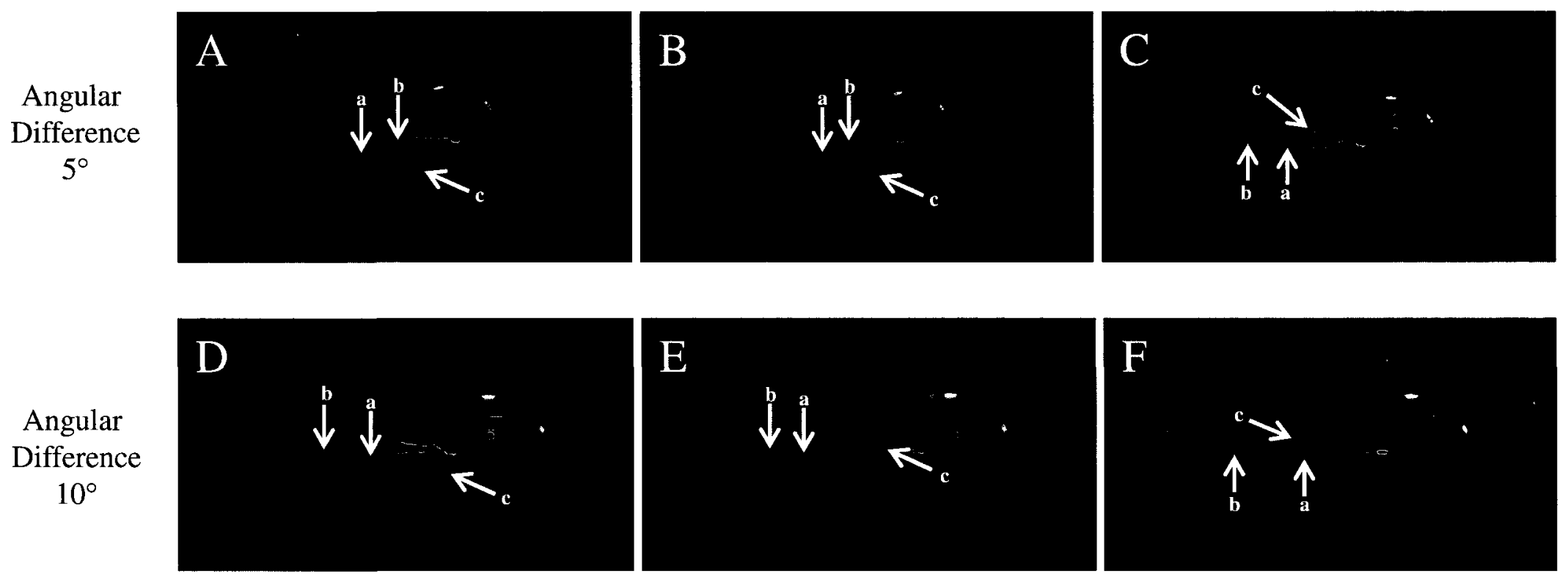

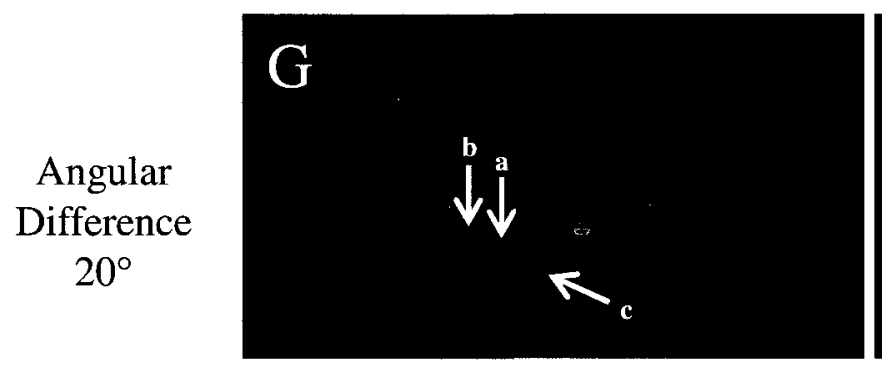

Separation Angle

$9^{\circ}$

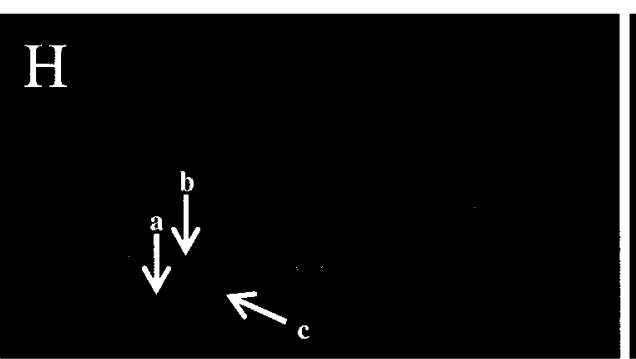

Separation Angle $17^{\circ}$

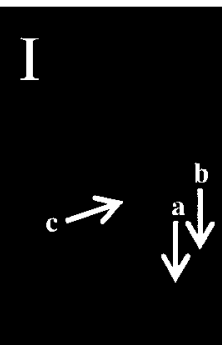

Separation Angle $30^{\circ}$

Figure 2.23. Dye-flow observations of wing-wing interactions of a rotating locust wing pair under varying angular differences and separation angles. Wings are at a configuration where the forewing has an angle of attack of $5^{\circ}$, the hindwing has an angle of attack of $30^{\circ}$. Figures (A)-(I) show both a primary LEV (a) and minor LEV (b) on the hindwing. There were no LEV's that formed on the forewing; therefore the dye flowed over the separation bubble of the hindwing (c). 
2.24). 3) As long as the angle of attack of the forewing was sufficient enough to cause turbulent flow to interfere with the LEV structures of the hindwing, again depending on the corresponding angles, see table 2.6 for specific examples, the flow of the forewing always resulted in the same effect on the hindwing LEV structures until the separation angles and angular differences were great enough to allow a separation bubble to form on the hindwing (Table 2.6, Figure 2.25).

It should be noted, that although in many cases the dye did not become entrained in a LEV structure on the hindwing as a result of the forewing, there were some instances where spanwise flow was observed and dye from the forewing travelled over an invisible separation bubble on the hindwing (Figure 2.21).

Detachment of a LEV is dependent on the angle of attack of the wing. The point at which a LEV detaches from the wing and becomes entangled in to the tip vortex is referred to as the detachment point $(\mathrm{Dp})$. The detachment points of the minor and primary LEV structures appear to be at the same point along the leading edge of the wing from one another, for example the primary LEV detaches at $65 \%$ of the total wing length of the hindwing when it is at an angle of attack of $30^{\circ}$ and the forewing is at an angle of $5^{\circ}$. The minor LEV also has a Dp point of $65 \%$ of the total hindwing length for this configuration. The detachment points remain relatively constant with the corresponding angles of attack of the wings regardless of the SA and AD. As the angle of attack increases and the flow surrounding the wing surface becomes more turbulent, the detachment point decreases. The detachment point of the LEV structures of the locust hindwing can be viewed in tables 2.5 and 2.6 . 

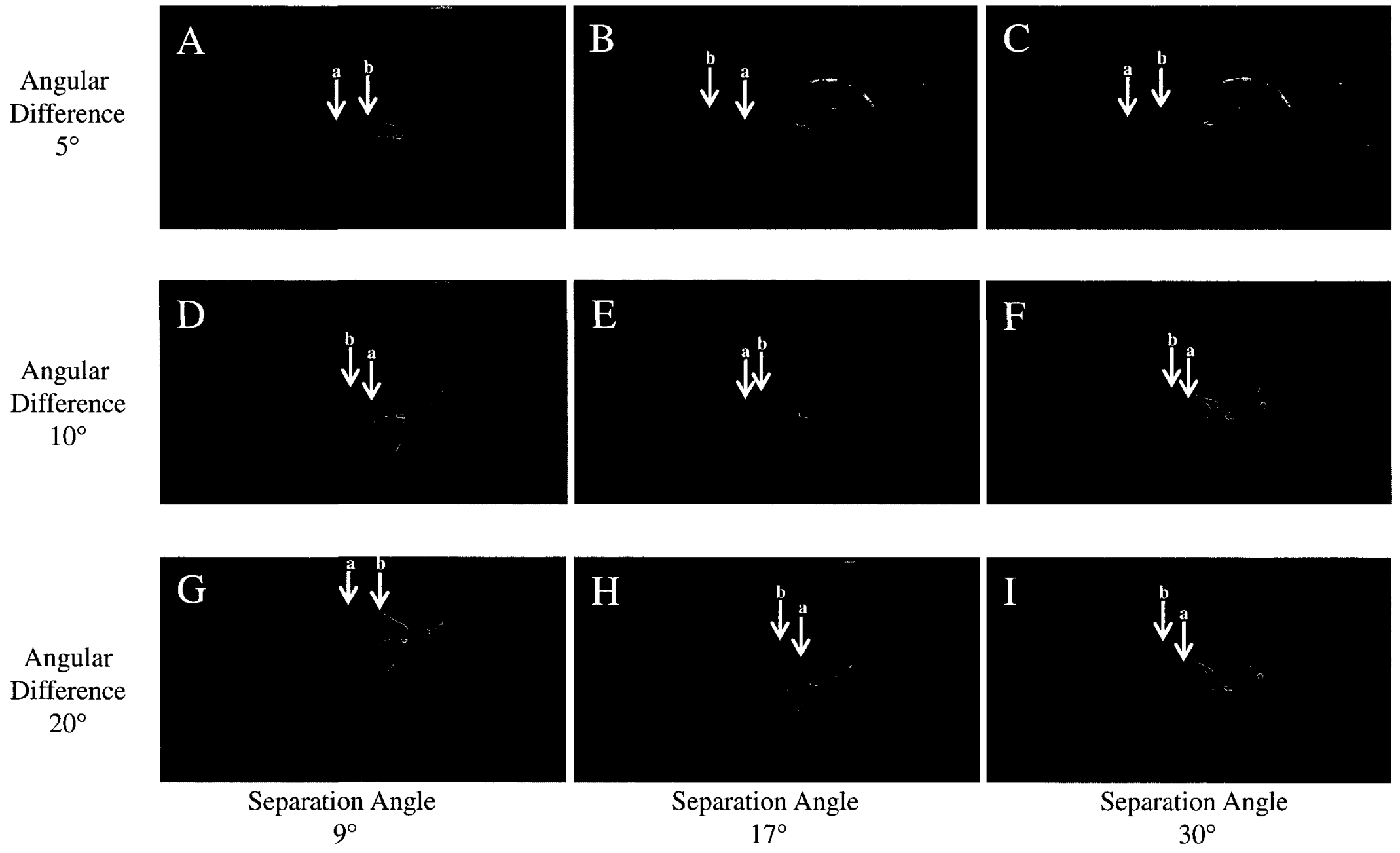

Figure 2.24. Dye-flow observations of wing-wing interactions of a rotating locust wing pair under varying angular differences and separation angles. Wings are at a configuration where the forewing has an angle of attack of $30^{\circ}$, the hindwing has an angle of attack of $15^{\circ}$. Figures (A)-(I) show both a primary LEV (a) and minor LEV (b) on the forewing. There were no LEV's that formed on the hindwing; therefore the dye flowed down the cord of the wing in a stream-like manner. 

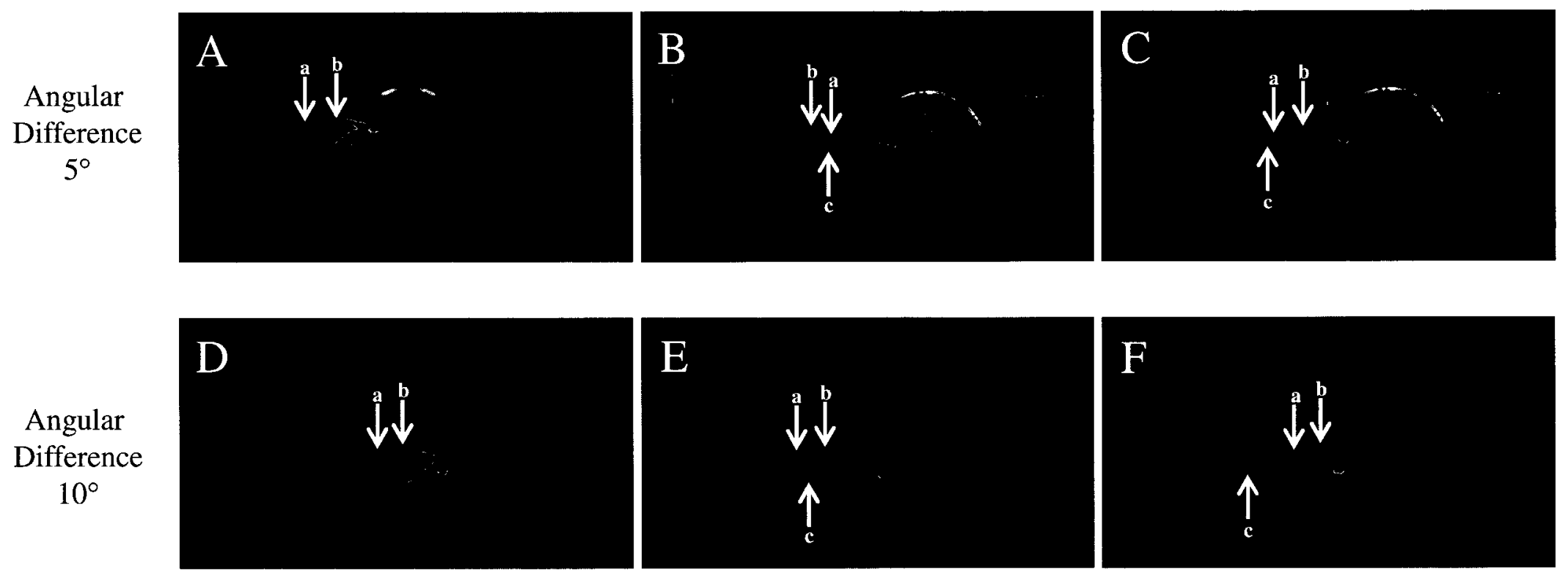

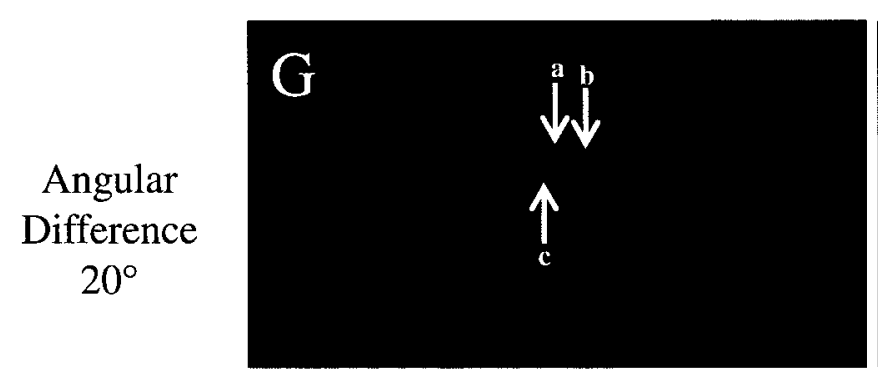

Separation Angle

$9^{\circ}$

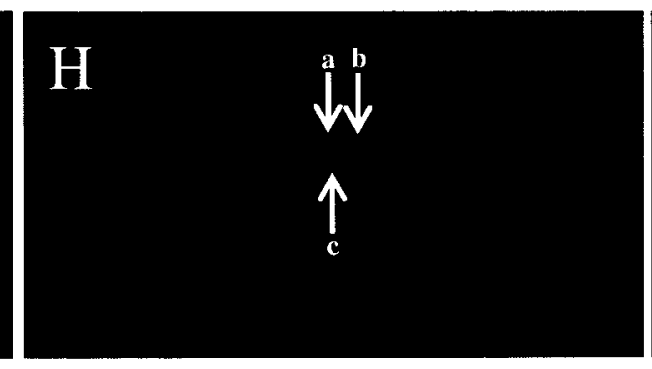

Separation Angle $17^{\circ}$

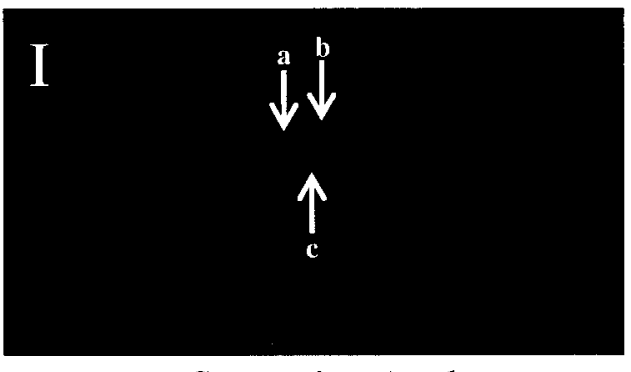

Separation Angle $30^{\circ}$

Figure 2.25. Dye-flow observations of wing-wing interactions of a rotating locust wing pair under varying angular differences and separation angles. Wings are at a configuration where the forewing has an angle of attack of $30^{\circ}$, the hindwing has an angle of attack of $30^{\circ}$. (A)-(I) show both a primary LEV (a) and minor LEV (b) on the forewing, and (B, C, E-I) show a minor LEV developing on the hindwing (c). 


\subsubsection{Volumetric 3-Component Velocimetry}

\subsubsection{Qualitative Analysis of Flow Structures}

Several wing configurations were employed using the V3V system to acquire further results to compliment the observations of the dye-flow experiments. Each configuration was time averaged over multiple rotations to eliminate random or background flow patterns of individual rotations.

The clockwise vortcitiy magnitude is visualized using isostructures which are represented by the blue tube-like structures seen in Figures 2.26, 2.27 and 2.28. The most substantial isostructures represent the LEV structures formed along the length of the wing from the wing base. For clarity purposes, isostructures representing counter-clockwise vorticity typically seen in the downwash for example, were removed from the V3V figures.

The LEV structures in the forewing were present for angles of attack greater than $0^{\circ}$, but increased in magnitude and velocity as the angle of attack increased. Dual LEV's were observed at angles greater than $15^{\circ}$.

The LEV structures on the hindwing were always present with the angles of attack greater than $0^{\circ}$, even with the presence of the forewing at various configurations; however, the vorticity magnitude is substantially less for the LEV structures on the hindwing when the interactions of the forewing are occurring, as opposed to when the hindwing was spun in isolation (Figure 2.29). 

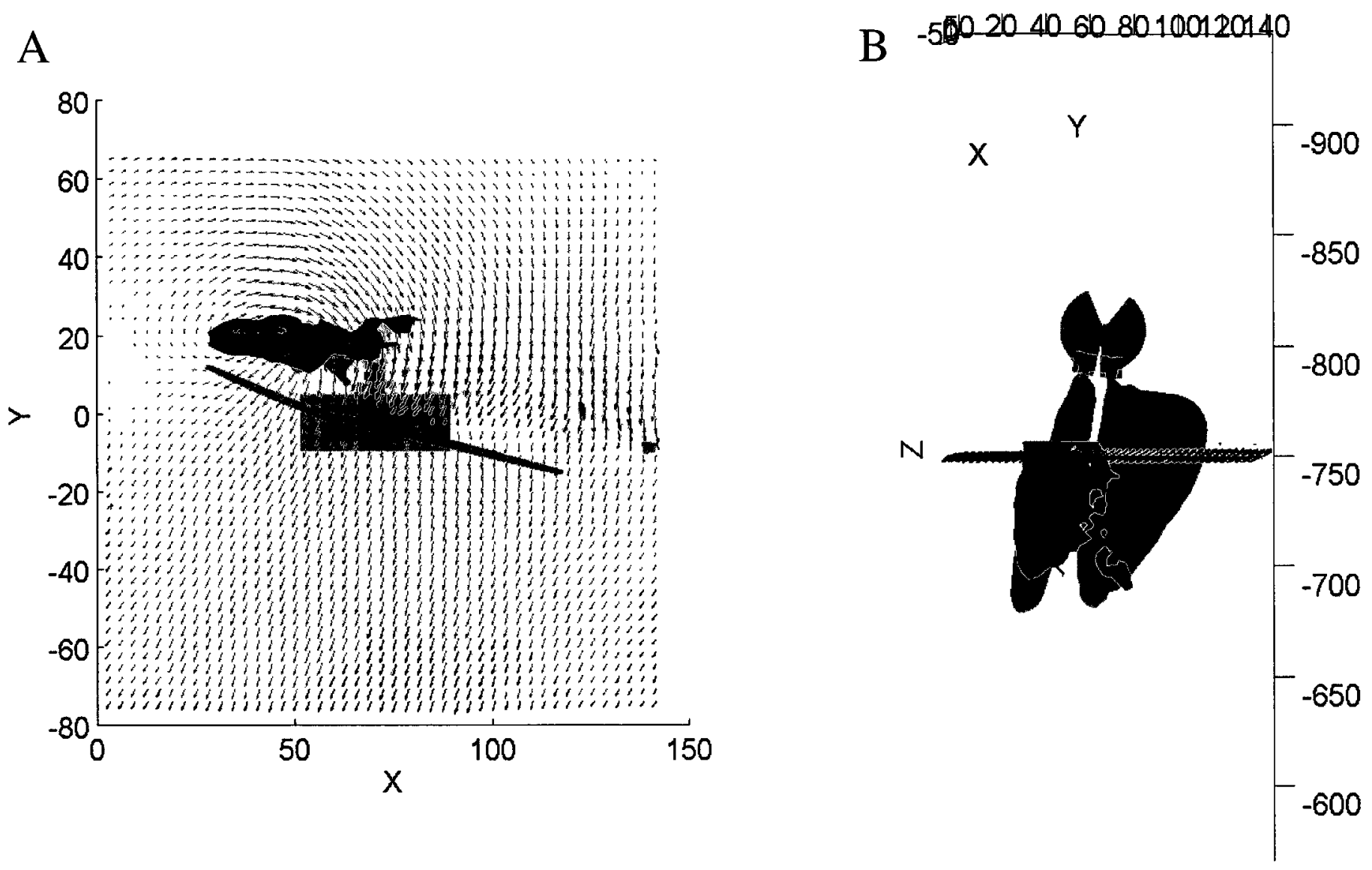

Figure 2.26. Calculated vorticity isocontours (isostructures) for a forewing at an angle of attack of $25^{\circ}$, the hindwing at an angle of attack of $15^{\circ}$, an angular difference of $5^{\circ}$, and a separation angle of $9^{\circ}$. Arrows are relative velocity vectors. (A) clockwise isostructures seen from the wing viewed tip to base (counter clockwise isostructures omitted for clarity). (B) isostructures viewed from above. The velocity vector plane is located at $r / R=0.4$. Vorticity is seen above the forewing but not the hindwing. Vorticity travels along the wing span toward the tip where it is shed at the tip vortex. 


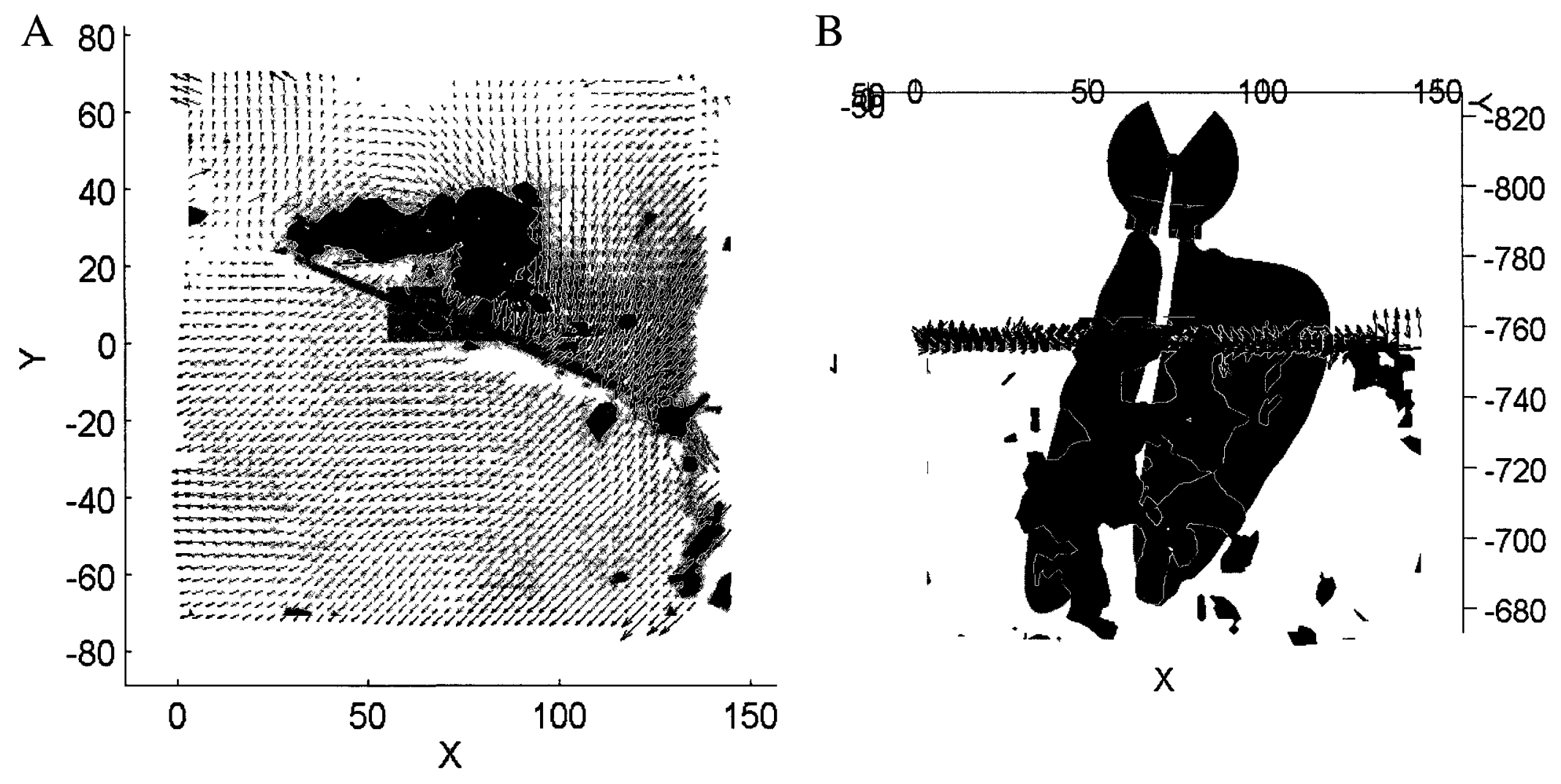

Figure 2.27. Vorticity isocontours /isostructures for a forewing at an angle of attack of $25^{\circ}$, the hindwing at an angle of attack of $25^{\circ}$, an angular difference of $5^{\circ}$, and a separation angle of $9^{\circ}$. The high angle of attack of the two wings increases the complexity of the flow field; however, a leading edge vortex is not observed above the hindwing despite the large angle of attack. (A) wing couple viewed from tip to base. (B) wing couple viewed from above. The velocity vector field is located at $r / R=0.4$. 

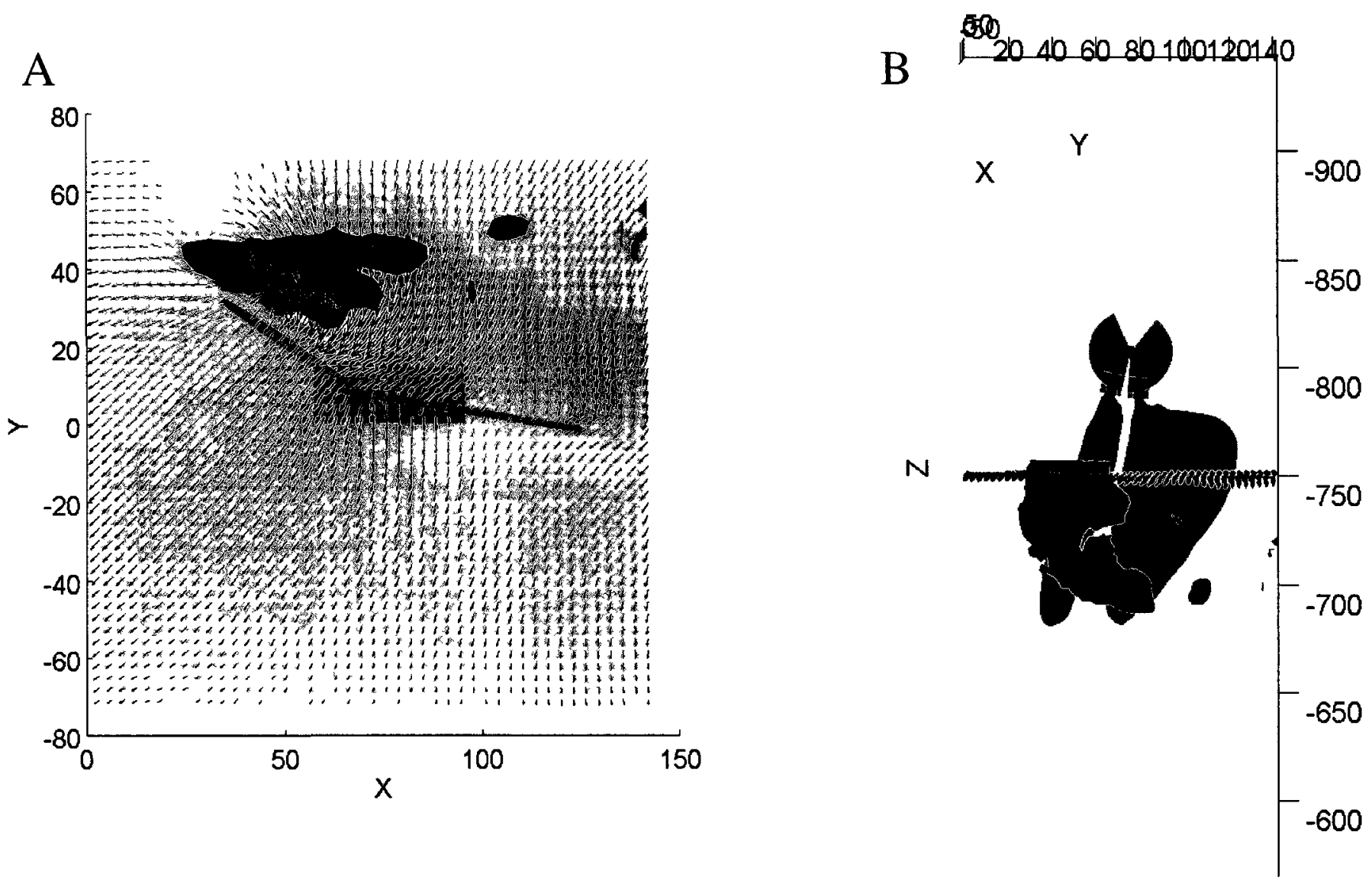

Figure 2.28. Vorticity isocontours / isostructures. The leading edge vortex formed above the forewing (A) is clearly seen feeding into a tip vortex for the rotation wing couple (B). No leading edge vortex is seen above the hindwing. The forewing is at an angle of attack of $30^{\circ}$, the hindwing at an angle of attack of $10^{\circ}$, the angular difference is $10^{\circ}$, and the separation angle is $9^{\circ}$. (A) shows the wings and isostructures viewed from the camera's perspective (end on), (B) is the view from above. The velocity vector field is located at $\mathrm{r} / \mathrm{R}=0.4$. 


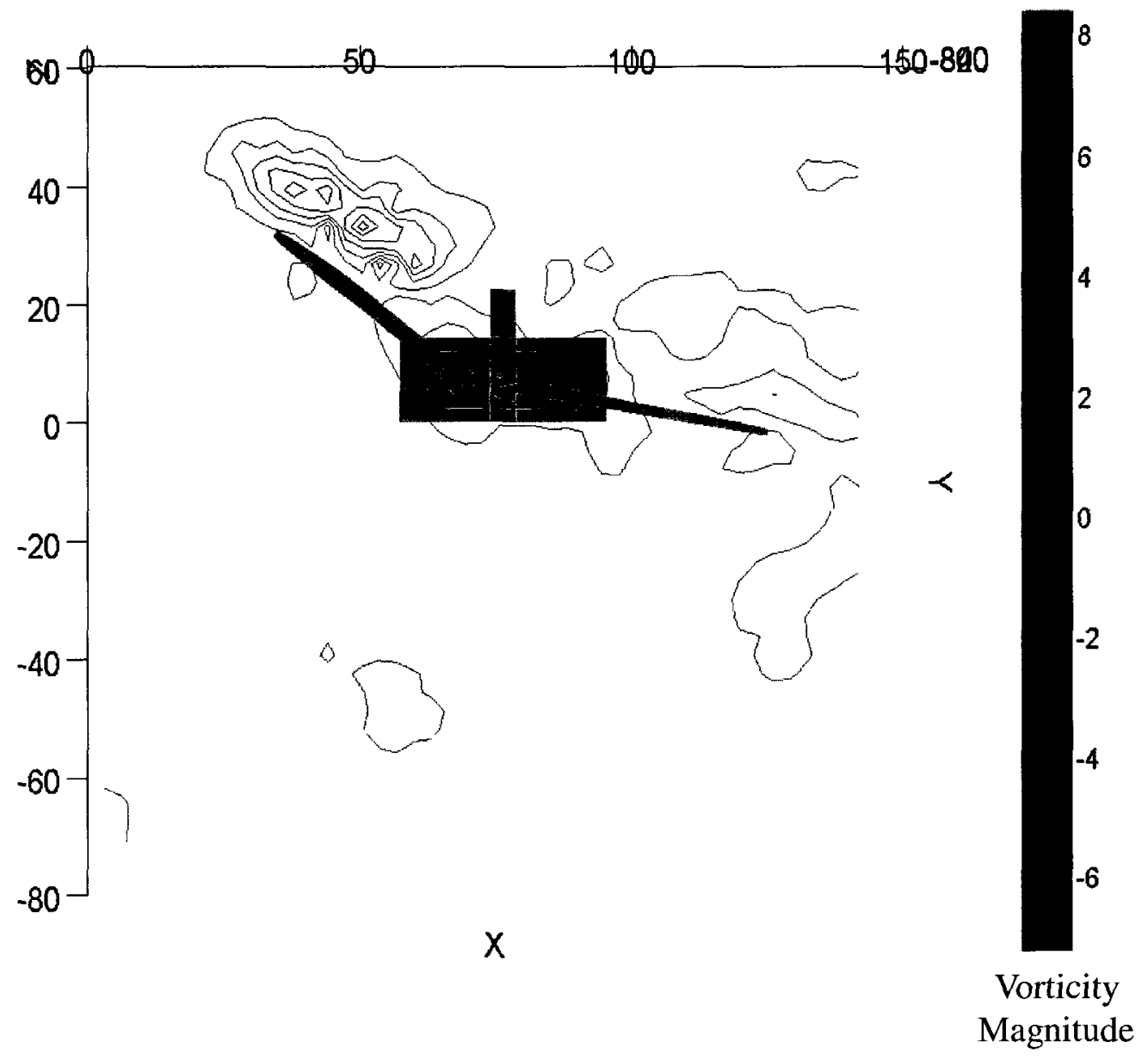

Figure 2.29. Vorticity contours observed for a wing pair (viewed along the wing span from tip to base). The forewing is at an angle of attack of $30^{\circ}$, the hindwing at an angle of attack of $10^{\circ}$, an angular difference of $10^{\circ}$, a separation angle of $9^{\circ}$ and a rotation speed of $11.4 \mathrm{~s}$. 


\subsubsection{Velocity Magnitude of the LEV Structures}

Preliminary data acquired using the V3V system allowed for quantitative measurements of flow structures on the rotating locust fore- and hindwing pairs.

A velocity contour slice was placed at $r / R=0.40$ the wing length from the base of the wing in each configuration. This was to ensure the ability to capture the velocity magnitude of the LEV structures prior to the detachment point where the LEV's become entrained in the tip vortex. This value was obtained by using the lowest $\mathrm{Dp}$ value observed in the dye-flow technique (Table 2.6).

Wing configurations with low forewing angles of attack (i.e. $5^{\circ}$ ) showed, as expected, low velocity magnitude values (Table 2.7). LEV structures on the hindwing with angles of attack higher than $15^{\circ}$ and forewing angles of attack lower than $5^{\circ}$ were able to produce strong velocity magnitudes (Table 2.7 ). As the angle of attack increases in the forewing, the velocity magnitudes decrease to values approaching zero in the hindwing, although they begin to increase with increasing separation angles and angular differences which is consistent with the observations seen in the dye-flow visualization technique (Table 2.7, Figures $2.30,2.31,2.32,2.33$ ). The velocity magnitude of the vorticity structures on the forewing are relatively consistent with the respective angles of attack regardless of the angle of attack of the hindwing, SA and AD, again, supporting the observations seen in the dye-flow visualization technique. 
Table 2.7. Summary of calculated velocity magnitudes of a locusts' wing pair using the volumetric 3-component velocimetry visualization technique. Various angles of attack of both the fore- and hindwings, as well as separation angles and angular differences were employed.

\begin{tabular}{cccccccc}
\hline $\mathrm{SA}\left(^{\circ}\right)$ & $\mathrm{AD}\left({ }^{\circ}\right)$ & $\mathrm{FW} \alpha\left(^{\circ}\right)$ & $\mathrm{HW} \alpha\left(^{\circ}\right)$ & $\begin{array}{c}\text { Velocity } \\
\text { Magnitude on } \\
\mathrm{FW}(\mathrm{m} / \mathrm{s})\end{array}$ & $\begin{array}{c}\text { Velocity } \\
\text { Magnitude on } \\
\mathrm{HW}(\mathrm{m} / \mathrm{s})\end{array}$ & $\begin{array}{c}\text { Rotation Speed } \\
(\mathrm{s})\end{array}$ & $\begin{array}{c}\text { Reynolds } \\
\text { Number (Re) } \\
\text { of Hindwing }\end{array}$ \\
\hline 5 & 9 & 0 & 15 & 0.0025 & 0.0105 & 11.8 & 4300 \\
5 & 9 & 5 & 20 & 0.012 & 0.0225 & 11.9 & 4300 \\
5 & 9 & 5 & 25 & 0.0075 & 0.034 & 11.8 & 4300 \\
5 & 9 & 15 & 5 & 0.019 & 0.005 & 12.1 & 4300 \\
5 & 9 & 25 & 15 & 0.021 & 0.0001 & 11.9 & 4300 \\
5 & 9 & 25 & 25 & 0.043 & 0.01 & 11.7 & 4300 \\
5 & 9 & 30 & 10 & 0.038 & 0.002 & 11.6 & 4300 \\
5 & 17 & 5 & 20 & 0.0055 & 0.022 & 11.6 & 4300 \\
5 & 17 & 25 & 25 & 0.026 & 0.006 & 11.9 & 4300 \\
5 & 17 & 30 & 10 & 0.042 & 0.018 & 11.5 & 4300 \\
5 & 30 & 5 & 20 & 0.0057 & 0.021 & 12.2 & 4300 \\
5 & 30 & 25 & 25 & 0.025 & 0.026 & 12.2 & 4300 \\
5 & 30 & 30 & 10 & 0.037 & 0.01 & 11.7 & 4300 \\
10 & 9 & 5 & 20 & 0.0145 & 0.019 & 11.7 & 4300 \\
10 & 9 & 25 & 25 & 0.028 & 0.019 & 11.5 & 4300 \\
10 & 9 & 30 & 10 & 0.036 & 0.004 & 11.4 & 4300 \\
20 & 9 & 25 & 25 & 0.04 & 0.028 & 11.8 & 4300 \\
20 & 9 & 30 & 10 & 0.028 & 0.013 & 11.9 & 4300 \\
\hline
\end{tabular}




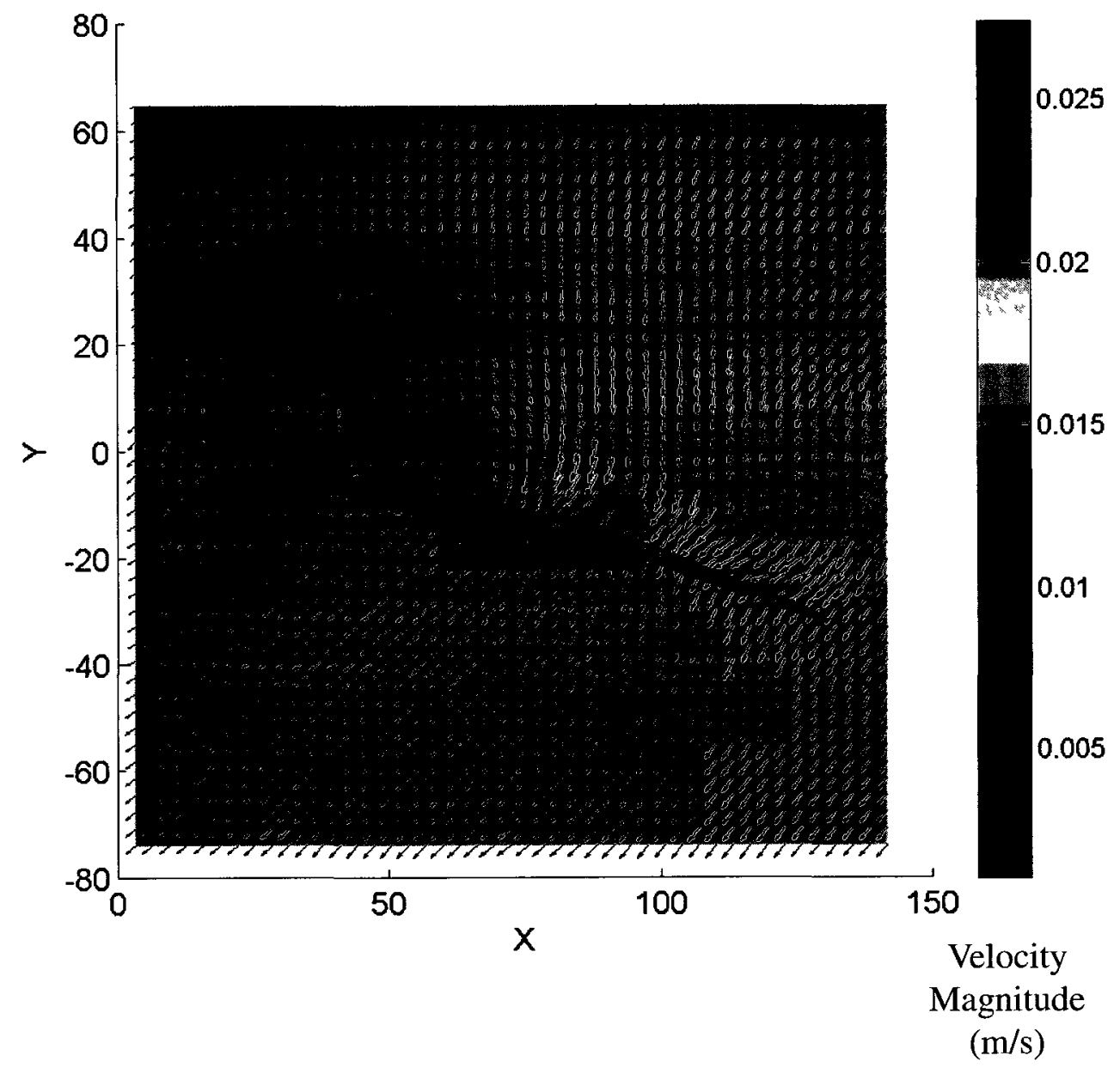

Figure 2.30. The flow field around a forewing-hindwing couple. Arrows represent local velocity in a 'slice' positioned at $r / R=0.4$. Colour contours depict the magnitude of the velocity (see scale to right). In this configuration, there is no LEV on the forewing or hindwing. The forewing is at an angle of attack of $5^{\circ}$, the hindwing is at an angle of attack of $20^{\circ}$, the angular difference is $5^{\circ}$, and the separation angle is $17^{\circ}$. 


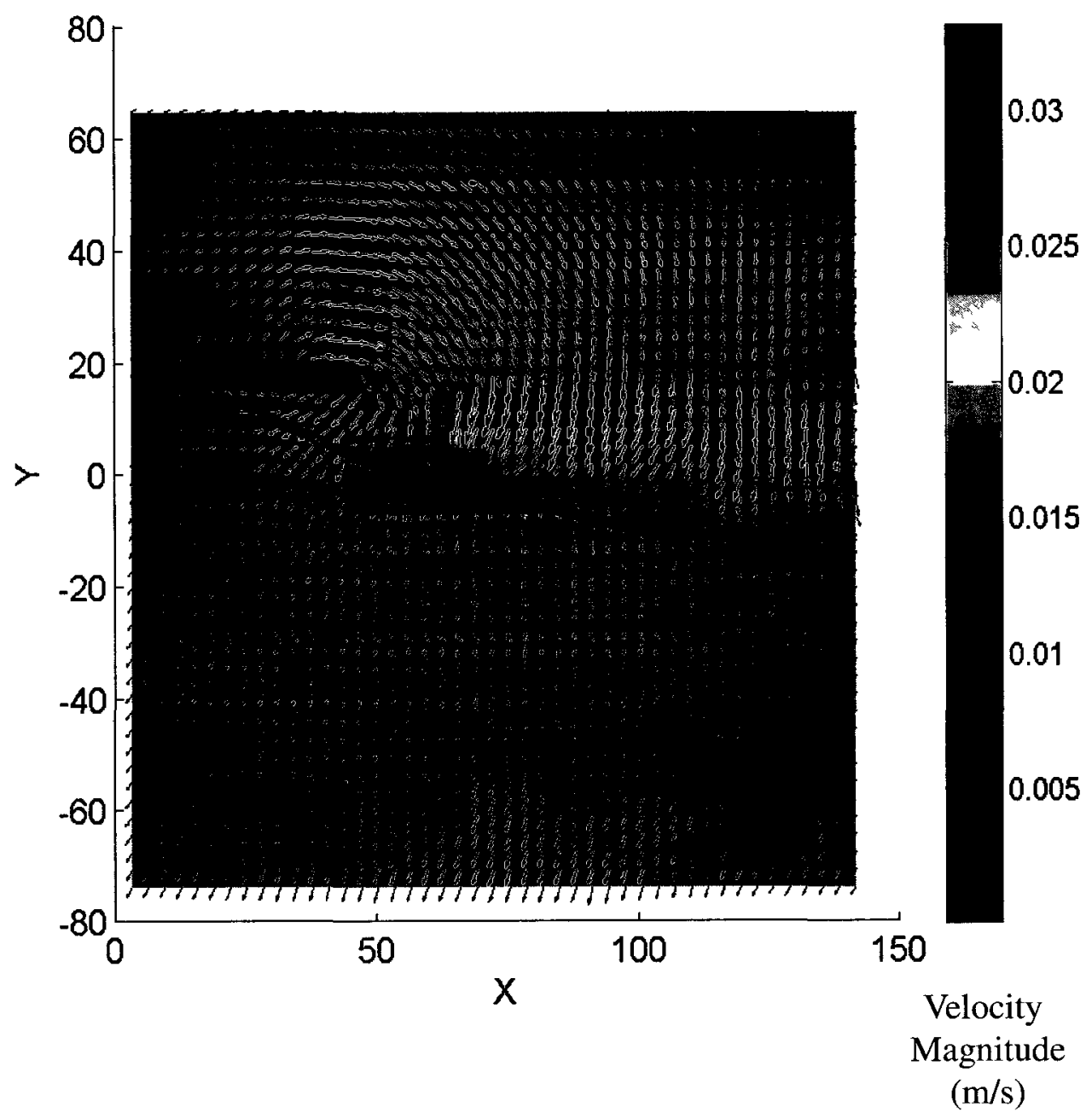

Figure 2.31. A leading edge vortex is found above the forewing (angle of attack $=25^{\circ}$ ) but not the hindwing in this wing couple. The forewing is at an angle of attack of $25^{\circ}$, the hindwing is at an angle of attack of $15^{\circ}$, the angular difference is $5^{\circ}$, and the separation angle is $9^{\circ}$. The flow field is centred at $r / R=0.4$. 


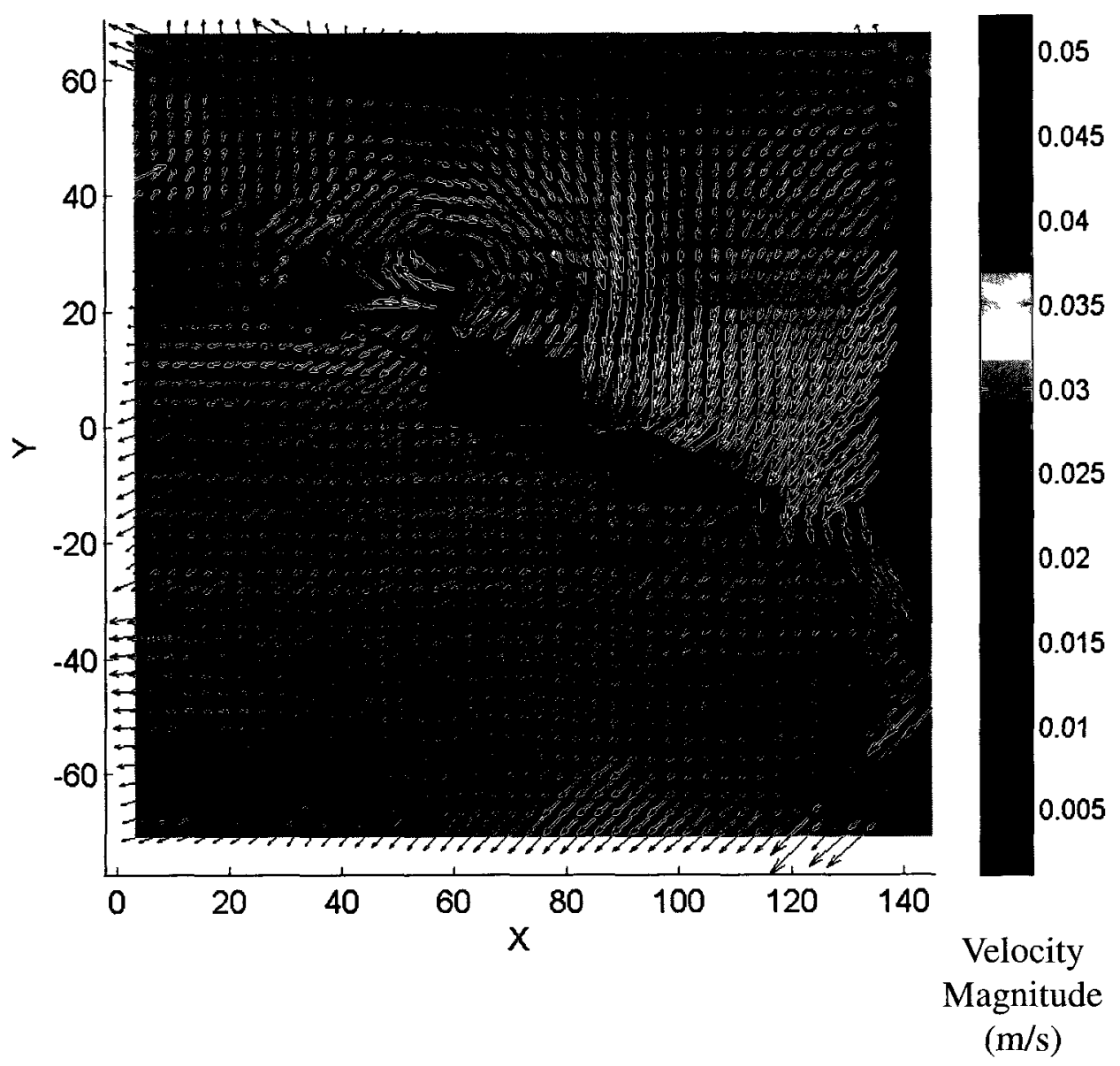

Figure 2.32. Flow field around a wing couple with each wing at high angle of attack (forewing angle of attack $=25^{\circ}$, hindwing angle of attack $=25^{\circ}$ ). No leading edge vortex is observed above the hindwing despite its high angle of attack. The plane is measured at $\mathrm{r} / \mathrm{R}=0.4$. The angular difference between the wings is $5^{\circ}$, and the separation angle is $9^{\circ}$. 


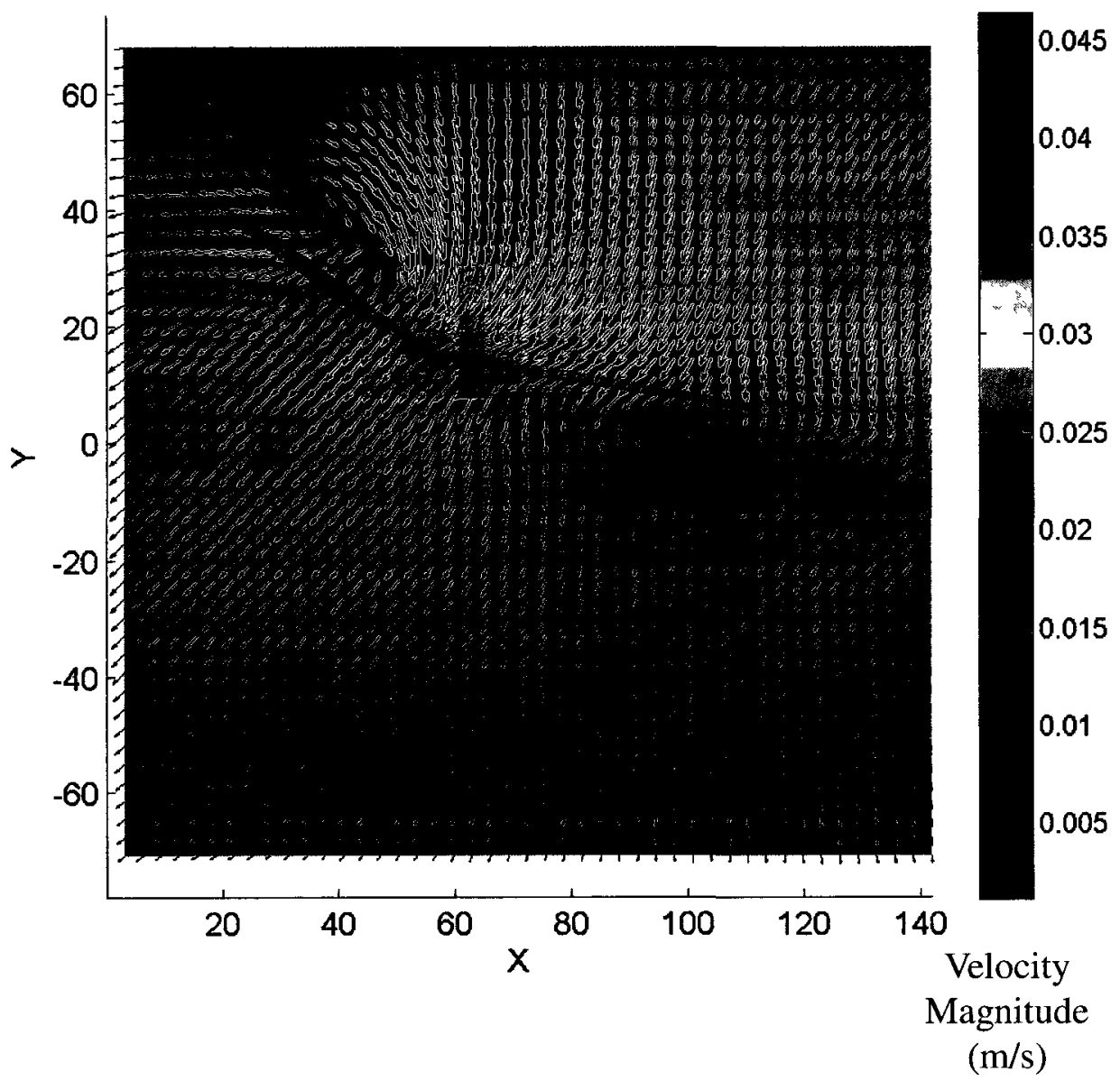

Figure 2.33. Flow field for a wing couple with the forewing at an angle of attack of $30^{\circ}$, the hindwing at an angle of attack of $10^{\circ}$, the angular difference is $10^{\circ}$, and the separation angle is $9^{\circ}$. The flow field is measured at $r / R=0.4$. 


\subsection{Discussion}

\subsubsection{Kinematics}

\subsubsection{Acoustic Startle Response}

Many nocturnal, flying insects exhibit an acoustic startle response (ASR) because they are susceptible to predation by insectivorous bats (Dawson et. al., 2004a, b). Examples include crickets, katydids, praying mantises and tympanate moths (Dawson et. al., 1997). Observations throughout the trials in this study showed that locusts exhibited typical acoustic startle response (ASR) behaviours consistent with the literature (Robert, 1989; Dawson et. al., 1997, Dawson et. al. 2004, Fauquier, 2008); these behaviours included abdomen dorsiflexion and deflection, hindleg extension, flight cessation and head roll. The majority of locusts throughout this study showed at least one or more of these characteristics after the high frequency stimulus was applied. I conclude that the acoustic stimuli used were therefore initiating genuine attempts to change direction by the locusts and thus that the subsequent kinematic changes in asymmetric wing depression, angular difference, separation angle and angle of attack were representative of locusts engaged in attempted steering maneuvers.

\subsubsection{Tethering Effects}


Tethering is an unavoidable necessity of any kinematics experiment in which high-resolution high-speed cinematography is employed. Unfortunately, tethering does influence the behavior of the insect under observation. For flight studies, the absence of an optical flow field, the interruption or inversion of afferent mechanosensory signals, and the lack of appropriate proprioceptive feedback have all been cited as effects of using rigid tethers. In this study, it was shown that wing beat frequencies recorded from a locust tethered with a rigid sternal tether did not significantly differ from those recorded from a locust tethered with a semi-rigid ventral tether (note. the sample size for the semirigid dorsal tether was small, $\mathrm{N}=4$ ). The possible effects due to the presence of a tether have been largely debated among the flight community (Fry et. al, 2004, Dawson et. al., 2007). The ventral tether allowed the locust to pitch, yaw, and turn in a desired direction where was the rigid abdominal tether limited the locust to abdominal and head movements as it was tethered to a fixed position. The ventral tether allowed for easily distinguishable observations of the acoustic startle response as seen in Dawson et. al., 2004 , free-flying locusts, where as assumptions based on characteristics observed were made on the intended behaviour for the rigid tether such as turn direction based on the direction of head roll and abdomen dorsiflexion.

Locusts overall exhibited no significant differences in their flight kinematics as a result of the tethering method; however, visual observations indicate some differences in overall performance of the locust. It was more difficult to secure the locust using the ventral tethering method in terms of proper placement and the size of the steel wire which was dependent on the size of the locust. This resulted in the insect being handled for a longer period of time, outside of the incubated container which could have potentially 
impacted the overall flight performance of the insect. Due to the flexibility of the plastic tubing (hence termed a loose tether), the locusts typically took longer than when secured to the rigid abdominal tether to find the steady air stream and enter in to a flight posture, if they were able to attain the flight posture at all. Although trials were not at a length in which the locusts would readily fatigue, there were more trials that were discarded using the ventral tether than the abdominal due to various reasons such as the insect flying with a bias to one side as a result of improper tether position, the insect never achieving the tucked flight posture, or simply not flying at all for unknown reasons.

A previous study completed in 2008 showed significant differences in the ASR behaviours as a result of three different tethering methods, a loose tether, a saddle (pronotum) tether, and the abdominal tether used in this study, but not in wing beat frequency (WBF) which is consistent with the results observed in this study. These results do not conclude that no tethering artefacts are present, but instead that the potential effects on the flying locust are not significantly different for the kinematic variables that are of interest for this study and that tethering does not interfere with the animal's initiation of an ASR.

I made detailed kinematic observations of tethered flying locusts for two reasons. First, it was important to establish the biologically relevant ranges of these kinematic variables during straight flight and steering to validate the models used for dye flow visualization and V3V studies. Second, the use of an acoustic stimulus (and the ASR) allowed me to determine if these variables change during attempted steering maneuvers. Wing-wing interactions are possibly a potent mechanism for generating steering torques, 
so it is a necessity to have accurate representations of kinematics so observe the effects on wing-wing interactions that can presumably be applied to real, free flying locusts.

\subsubsection{Wing Stroke Amplitude}

In terms of lift, the prevailing view in the literature is that the hindwings are responsible for the majority of the force production the forewings do very little in that respect (Jensen, 1956). The forewings exhibit most of the change in kinematics (Robertson et. al., 1996, Dawson et. al, 1997, Robertson \& Reye, 1992, Robertson \& Johnson, 1993, Dawson et. al., 2004) and thus are reported to be key players in effecting steering maneuvers. At $1 / 3$ the downstroke, the hindwings are approximately at $0^{\circ}$ relative to the horizontal and the forewings are exhibiting changes in asymmetric wing depression and WSA (Dawson et. al, 1997).

An increase in WSA on one side of the locust would indicate a turn in the opposite direction, meaning the side with the increase would be considered the contralateral wing due to the fact that one side of the locust is producing more force than the other causing the insect to rotate. Alternatively a decrease in WSA on one side of the locust would indicate a turn in the same direction, therefore being the ipsilateral wing since force production would decrease on that side. If the WSA increased on both sides, this would indicate a climbing pattern or an increase in velocity as more forces would be produced overall, and a decrease in WSA would indicate a decrease in velocity, or more likely, a dive as a form of evasive maneuver where force production would be reduced. 
The WSA increased significantly for both ipsilateral and contralateral forewings after an acoustic stimulus was introduced. This result indicates that WSA played a role in the ASR of the locusts, consistent with the findings in the literature mentioned above. The increase suggests that the locust was either increasing its velocity to escape the predator-like sound, or climb in elevation, again, to evade the stimulus.

\subsubsection{Forewing Asymmetry}

Roll torques, responsible for steering, are generated when the lift and/or thrust production is changed asymmetrically on one side of the locust (Dawson et. al, 1997). A change in WSA on one side of the locust corresponds to a forewing asymmetry (FWA), but FWA is a comparison of both forewing angles relative to the vertical axis (Figure 1.5), where as WSA is evaluated from peak of the upstroke to peak of the downstroke (Figure 1.3) and each wing is measured independently irrespective of axes, and can include the hindwings; therefore a change in WSA is not always indicative of FWA.

Shoemaker \& Robertson, (1998) describe two types of steering, correctional and intentional steering. Intentional steering is the focus of FWA where the locust is actively changing its flight path in the direction of the desired location (i.e. as part of an ASR or perhaps obstacle avoidance, etc.). Correctional steering is described as being a passive response of the locust to maintain a straight and steady flight path and FWA are either small or absent (Dawson et. al., 1997).

In terms of turn direction, off all 14 trials, $36 \%$ of locusts steered right towards the acoustic stimulus and $64 \%$ of locusts steered left away from the stimulus. Robert, 
(1989), concluded that locusts deflect their abdomens and hindlegs away from the source of sound, but a previous study that I have conducted (2008) as well as the results stated above show no preference in turn direction with a speaker in a position posterior and to the right of the locust.

The results show significant differences in FWA post-stimulus irrespective of the turn direction. Due to the fact that significant differences exist, these results indicate that large FWA's developed post-stimulus, which in turn indicates intentional steering.

\subsubsection{Separation Angle}

Some insects such as moths will advance the leading edge of one wing and retract the leading edge of the other during an attempted turn (Kammer, 1971, Roeder, 1966). Thus locusts may also modulate separation angle for steering. This study showed that there were no significant differences in separation angle (SA) between the ipsilateral and contralateral wing pairs both pre- and post-stimulus. This means that the SA remains relatively constant throughout the flight of a locust even during evasive maneuvers. This suggests that SA should not have an effect on wing-wing interactions of flying locusts since real locusts do not change the angle as part of an ASR.

\subsubsection{Angular Difference}

There has been much discussion about angular difference (AD) and phase relationships playing a significant role in wing-wing interactions (Lehmann et. al., 2005, 
Wang \& Sun, 2005, Wang \& Russell, 2007, Deng \& Hu, 2008). In locusts, the hindwing leads the forewing by a phase relationship of $10-30^{\circ}$ throughout the downstroke (Figure 2.34)(Wilson \&Weis-Fogh, 1962, Robertson \& Reye, 1992), which translates to varying angular differences between $20-50^{\circ}$ throughout the downstroke. Dragonflies on the other hand exhibit varying phase relationships depending on the flight characteristics. For example, during hovering, there will be a phase relationship of $180^{\circ}$ between the forewings and the hindwings so that equal amounts of lift and drag will be produced throughout the upstroke and the downstroke (Wang \& Sun, 2005). The kinematic analysis shows the changes in $\mathrm{AD}$ of a flying locust as a result of an acoustic stimulus for the first time in a three-dimensional system. There were no significant differences between the ipsilateral and contralateral wing pairs pre- and post-stimulus. These results suggest that FWA is the important kinematic variable for steering, not AD. FWA and AD correspond to one another; however, as mentioned above, FWA incorporates both forewings to calculate the asymmetric wing depression where are the $\mathrm{AD}$ incorporates the forewing and the hindwing and therefore, FWA doesn't necessarily mean AD, it depends on the position of the hindwings. Dawson et. al., (1997) hypothesized that stronger motor pattern recordings would indicate that the locust is under active control of the particular kinematic variable in questions, therefore a prediction would be that FWA establishes the forewing-hindwing interactions, not $\mathrm{AD}$, and is under active control of the locust. 


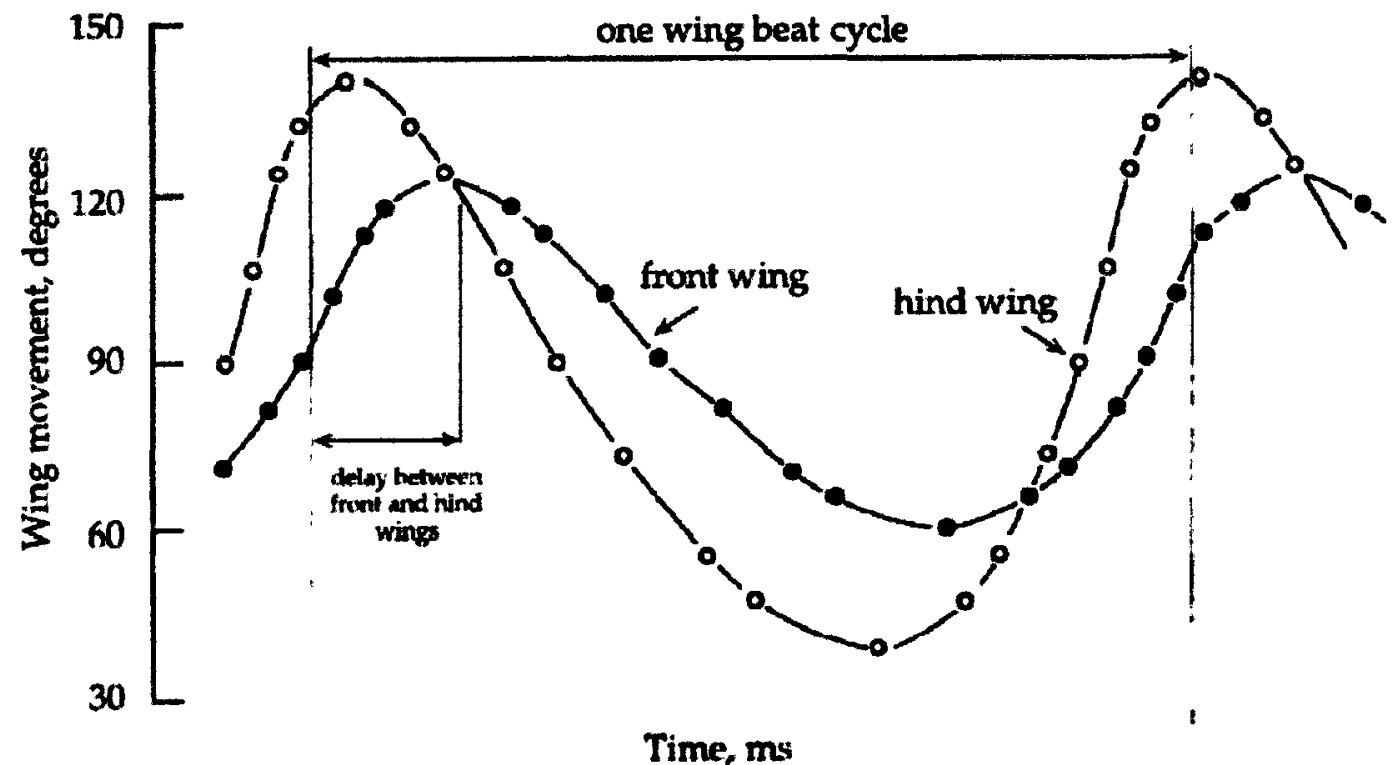

Figure 2.34. The wing tip path of a locust forewing and hindwing during flapping. The hindwing stroke leads the forewing stroke with a time difference typically between 5$10 \mathrm{~ms}$. Modified from Weis-Fogh (1956) as presented in Burrows (1996). 


\subsubsection{Wing-Wing Interactions in Rotating Wing Models}

\subsubsection{Overall Bulk Flow}

I did not observe instances in which the flow leaving the forewing re-attached to the hindwing as hypothesized in (Cebulski, 2010). Some insects such as butterflies, physically couple their wings together to increase the surface and effectively fly with two functional wings as opposed to four individual wings. This increases lift production by creating a greater pressure gradient as the flow above the surface of the wing must travel faster than the flow below the surface of the wing (Ellington, 1984). A reasonable hypothesis would be that all four-winged insects would attempt to mimic this situation in a variety of ways to potentially increase lift forces, however; if the flow is not able to reattach to the hindwing, locusts must rely on different kinematics to optimize lift production. This observation is limited to the wing configurations tested and the placement of the dye on the wing, therefore it is a possibility that this phenomenon may in fact play a role in flying locusts and was not observed in this study given the particular angles of attack, although various configurations of biologically relevant angles of attack were tested and the flow from the forewing was never viewed to re-attach, so the more likely possibility is that perhaps the placement of the dye did not allow for tracers to flow in the areas needed to visualize the re-attachment. As the hindwings are primarily responsible for lift production, and the forewings being widely responsible for steering, results in this study may appear to be contradictory as the forewing impedes and sometimes inhibits LEV structures from forming on the hindwing for what has described 
to be biologically relevant angles of attack, so the question to ask would be, how is the overall lift being produced in a flying locust? There are several possibilities to provide insight in to these questions. First, although the configurations in this study represent biologically relevant angles of attack in locusts, the majority of these configurations represent locusts during steering flight after an ASR. Goth, 2010, shows angles of attack of the forewing to be lower during straight, forward flight (i.e. $>\sim 20^{\circ}$ ) and presumably, higher hindwing angles of attack which would minimize the interaction of the forewing on the hindwing allowing for greater lift production. Some of the wing configurations support this hypothesis where the angle of attack of the forewing is equal or lower than the hindwing (i.e. forewing at $\propto 15^{\circ}$ and hindwing at $\propto 20^{\circ}$ ) (Table 2.6). Further analysis conducted with the V3V system using similar configurations also supports this hypothesis. Another possibility is that the wing models in rotation provide a velocity gradient along the wing, where the wing base has a low chord-wise flow and the wing tip has a much greater chord-wise flow as the velocity along the length of the wing increases (Figure 1.7). This case would describe a locust that is steering or climbing along a steep vertical incline similar in a take-off. A locust in straight forward flight would have approximately the same magnitude of chord-wise flow at the base of the wing to the tip of the wing (Figure 1.7). The differences in the chord-wise flows of the rotating wings would result in smaller magnitudes of spanwise flow and in turn affect the magnitude of the separation bubble. Lastly, it is likely that the contradictory results obtained in this study are a combination of both possibilities mentioned above due to the fact that there's no denying a stronger vorticity magnitude on the hindwing when spun in isolation than when paired with the forewing. 
The results do not imply that these interactions are not likely to occur in locusts, but it is unlikely that locusts exhibit these results in straight, forward flapping flight. These results more likely depict a locust during take-off, climbing flight, and steering maneuvers as often seen in the ASR.

Dawson et. al. (1997) observe that the forewings are primarily responsible for the steering maneuvers in locusts and are actively controlled. While steering, the lift on the hindwing would need to be minimized on one side of the locust to initiate a roll torque; therefore, the results of this study support the theory that a locust can actively decrease the angular difference between the forewing and hindwing to impede the vorticity magnitude of the separation bubble on the hindwing.

Kutsch and Fuchs (2000), describe locusts' flight patterns during take-off and steep vertical climbs where the locust needs ascertain some forward velocity (during take-off they jump to gain that velocity) followed by the forewing exhibiting very large separation angles and angular differences to achieve adequate lift forces to sustain flight. With the separation angles and angular differences at angles beyond what was observed in this study, it is possible that the locust is essentially creating enough distance between the wings that they are all functioning as separate entities to produce lift without the hindrance of forewing interactions on the hindwing.

Reasons for discrepancies among forewing angles of attack in locusts could be a result of a confounding issue with capturing angle of attack kinematics in real flying locusts which is tethering. When a locust is tethered in a stationary position in the air stream, it is incapable of repositioning its body angle, and therefore results may not accurately depict a body angle that a real locust would exhibit during free flight. This 
may in turn affect the geometric angle of attack which is defined as the angle of the wing cord relative to the air stream.

Results from the V3V trials support the findings of dye-flow that re-attachment does not occur on the hindwings, but rather the vorticity created by the LEV structures on the forewings are sufficiently great in velocity magnitude to encompass almost the entire surface area of the forewing and hindwing resulting in a large clockwise-rotating flow over both wings (Figure 2.33). As shown in Figure 2.29, there is some vorticity magnitude occurring on the leading edge of the hindwing at angles greater than $0^{\circ}$, but it is so low in magnitude that LEV structures do not appear when looking at the velocity magnitudes as the velocity of the vorticity magnitude of the forewing completely overpowers that of the hindwing for an overall resultant velocity magnitude following the direction of the flow on the forewing.

As previously mentioned, dye-flow visualization is restricted by the placement of the dye and it can often be difficult to view the entire gross flow structures involved in the rotating wings, where as the $\mathrm{V} 3 \mathrm{~V}$ system captures the entire area and can also quantify the flow fields. The V3V system revealed additional vortex structures, both clockwise and counter-rotating structures, as well as a counter-rotating downwash flow that were not visible in the dye-flow technique. V3V is more sensitive at detecting vorticity as it calculates based of relative velocity of local regions of flow. Dye-flow visualization of vorticity is dependent on the magnitude (low pressure) of the vortex, and placement of the dye (ability of the vortex to entrain the dye crystals).

An ideal situation would be to create a dye-rake to be implemented in to the dyeflow visualization as seen in Cebulski, 2010, to allow for more flow structures to be 
visualized in wing-wing interactions. Preliminary designs of rakes were attempted in the beginnings of this study, but without success. Constructing a dye-rake is challenging for trials involving wing-wing interactions because the majority of the area of interest is in on the surface of the hindwing; therefore a rake must be placed between the forewing and the hindwing to visualize the flow structures in this area without impeding the flow dynamics in surrounding areas due to the structure of the rake. If a rake were to be positioned in front of the forewing, the forewing would entrain all of the dye rendering it impossible to view any flow structures on the hindwing.

\subsubsection{Leading Edge Vortex}

The LEV has been shown to be the greatest contributor to lift production (Ellington et. al., 1997, van den Berg \& Ellington, 1997, Michelson \& Naqvi, 2003). Due to this fact, the LEV was a key area of interest for this study, specifically how the LEV on the hindwing is affected by the interactions of the forewing.

The literature describes LEV formations on various wing types, i.e. fruitfly, dragonfly, etc.; however, Lu et. al, (2006) showed that the LEV structures are insensitive to $A R$ and is dependent on angle of attack of the wing and Reynolds number; therefore, it can be assumed that the results obtained in this study can be applied to any flat plate wing models with similarly scaled Reynolds numbers and corresponding angles of attack.

LEV's begin to visibly form at angles of attack of $10^{\circ}$ or greater which is consistent with the literature (Lu et. al, 2006, Cebulski, 2010, etc.). Lu et.al., (2006) stated that dual LEV structures became visible at angles of attack greater or equal to $30^{\circ}$, 
however, I observed dual LEV's at angles between $20-25^{\circ}$ and greater when the wings were spun in isolation and on the forewing when spun together with the hindwing in dyeflow visualization. They were evident in angles much lower than that when using the V3V system $\left(\sim 15^{\circ}\right)$. This discrepancy may be due to the fact that Lu et. al. observed flapping wing models where the LEV structure is shed and reformed at the stroke reversals, where as in this case, rotating wing models allowed for a continuous simulation of the downstroke resulting in prolonged LEV attachment making this an ideal situation for observing the structures. Previous literature described the LEV's as a single entity, a large conical structure spiral outwards to the wing tip from the base of the wing (Ellington et. al., 1996, Lui et. al., 1998, Birch \& Dickinson, 2001, etc.). This discrepancy may be due several factors; 1) in the past, technology available has limited researchers to clearly visualize flow structures involved in moving objects, or objects placed in a stationary position in a flow stream whether it be air or fluid (i.e smoke or dye visualization). For example, low-resolution high-speed cinematography (similar to the technique used for the kinematics section of this study) can produce indistinct images, as well as low spatial resolution occurred in dPIV techniques, which rendered such detailed flow structures unclear, and perhaps the minor and primary LEV structures indistinguishable from one another. It is only recently where advances in technology, such as high-definition cinematography, have allowed researchers to clearly visualize the structures forming on the wing. 2) The majority of the studies have been conducted on flapping wing models where the LEV's are continuously shed. If the portion of the wing being analyzed was captured at particular times during the downstroke when the LEV's were being shed, they may have been overlooked. 3) As stated in Lu et. al., (2006), when 
using PIV laser-sheets in previous studies such as Birch et. al., 2003, depending on the angle of attack, the minor and primary vorticies, rather than being in the same plane as the wing (i.e. $x, y$ or $z$ plane), one or both of them may have been in between planes different than the wing and could not be illustrated by sectional sheets and therefore mistakenly be interpreted as not being present at all. 4) There may potentially exist substructures or micro-vortices in the space between the minor and primary LEV structures (hypothesized by Lu et. al., 2006 and Cebulski, 2010), resulting in the tracer flowing over all structures and resembling a single conical entity as opposed to being entrained in each of the various structures, depending the vorticity magnitude and again, the placement of the dye. The V3V system should be capable of detecting such micro-structures; however, the threshold for viewing them must be set to an extremely high value therefore the area of interest must be clearly defined, otherwise the magnitude of the minor and primary LEV structures will overpower the structures rendering them invisible.

The overall characteristics of the LEV structures appear to coincide with the literature where the LEV increases in diameter from base to tip until it detaches and becomes entrained in the tip vortex, and as the angle of attack increases, the angle between in the minor and primary LEV also increases and the detachment point decreases and detaches closer to the base of the wing (Lu et. al, 2006, Cebulski, 2010).

The results show that the LEV structures on the forewing appear to be unaffected by interactions of the hindwing. Studies focusing on the LEV structures in locusts are sadly lacking, but these results are supported by the findings of Cebulski, 2010. The detachment point and appearance of dual LEV's on the rotating forewing paired with the hindwing are consistent with the results of Cebulski, 2010, when the forewing is rotated 
in isolation. Since the hindwings are primarily responsible for the lift production and always lead the forewing with a constant phase relationship in locusts, I hypothesize that interactions between the wing pairs are solely affecting the flow structures on the hindwing of a locust and should therefore be termed forewing interactions on the hindwing as opposed to wing-wing interactions. This hypothesis would not hold true for insects that are capable of changing their phase relationships between the forewings and hindwings. Dragonflies for example, can alter the phase relationship between the wing pairs, and when they're hovering, they have a phase relationship of $180^{\circ}$ between the fore- and hindwings, so theoretically, with every change in upstroke and downstroke, and the interactions would change on the wings and affect both the forewings and the hindwings.

Interactions from the forewing are occurring on the hindwing, but to what extent these interactions have on free flying locusts remains unclear. This study shows that it is certain the flow structures on the forewing affect the development of the LEV structures on the hindwing, but do other interactions and flow structures still exist on the hindwing? Preliminary unpublished data conducted by Dawson et. al., 2007, showed the possibility that the development of LEV structures on the hindwing may only in fact be delayed along the length of the wing rather than inhibited as a result of the interactions from the forewing. This hypothesis cannot be ruled out by the results of this study for the following reasons due to the fact that dye was only placed at the base of the wing, so if a LEV failed to develop or was too weak to entrain the dye, the possibility of a LEV developing further along the length of the wing would not have been visible. The results of the V3V wing configurations show no evidence to support the hypothesis that LEV 
structures develop further along the wing; however, this may be a result of the time averaging. As stated in the methods section, several rotations of the same configuration were compiled together to eliminate spurious flow structures, but perhaps the delayed development of the LEV structures are so erratic that they were mistaken for 'noise' and eliminated from the gross flow structure. 


\section{Chapter 3 - Conclusions and Future Directions}

Although this study shows for the first time preliminary findings in locust wingwing interactions, still more research needs to be done to observe the possibility of other flow structures involved, and how these interactions affect real, free flying locusts in both straight, forward flight and steering flight.

For the first time, accurate depictions of angular difference, separation angles and wing stroke amplitudes are seen in 3-dimensions in flying locusts. These kinematics would presumably be irrespective of stroke plane and body angle during flight as that would simply represent a perspective issue which is irrelevant in 3-dimensional analyses (Figure 2.1). Goth, 2010, was the first to depict angles of attack in loosely tethered locusts in 3-dimensions. This provided supporting evidence of biologically relevant angles of attack observed in flying locusts; however, it remains unclear whether tethering artefacts played a significant role in the results of those angles. Accurate measurements of the angle of attack of the hindwing is still lacking as it is difficult to measure given the camber of the wing, but is necessary to evaluate the effect of other parameters in wingwing interactions. Accurate depictions of hindwing angle of attack during forward flight and steering need to be examined.

Further studies on varying wing configurations representing straight, forward flight as opposed to steering and steep ascent in real free flying locusts need to be observed. Many of the studies on flying locusts involve looking at the acoustic startle response (Dawson et. al., 1997, Dawson et. al., 2004, Fauquier, 2008, Goth, 2010, etc.). Although observations are made of straight flight prior to the stimulus being applied, emphasis is placed on the changes in kinematics post-stimulus. 
To improve the accuracy of results in dye-flow visualization and PIV methods, not only will it require more accurate angles of attack as mentioned above, but ideally constructing a more precise representation of locust wings would be beneficial as opposed to using flat plates as wing models. Although the flat plates were constructed based on biologically relevant Reynolds number and incorporated the $A R$, leading and trailing edge of a locust and overall wing shape, wing camber and wing surface were not incorporated and would no doubt have an effect on the flow structures, the question is, would this effect be significant? Another area of improvement would be the construction and use of dye-rakes in dye-flow visualization, which would benefit in terms of viewing more structures aside from the LEV structures in wing-wing interactions.

Dye-flow visualization and studies using V3V have allowed for viewing the overall flow structures on the forewings and hindwings, as well as some preliminary measurements on flow velocity. $\mathrm{V} 3 \mathrm{~V}$ even allows for the opportunity to measure vorticity magnitude, but these observations and measurements are difficult to translate directly in to lift production and drag forces, which is necessary to calculate accurate lift production. Exploring the area of how kinematic variable affect the flow structures, which in turn directly impact lift and drag forces will benefit in our understanding of wing-wing interactions across all species of insects.

A confounding issue when studying flow structures in moving objects is the ability to visualize them. There have been several discrepancies and new findings in recent years (i.e. the presence of dual LEV's). New advances in technology have clarified these discrepancies and allowed for new discoveries. Cebulski, 2010, mentioned how the use of a dye-rake and high-definition camera was required to view and document the 
presence of dual LEV structures, which may or may not impact results obtained in earlier studies where such technology was not available. Developing a standard for visualization techniques would be ideal to equally and accurately compare aerodynamics among varying wings, insect species, and even other animals, although admittedly, is improbable as technology is continuously evolving.

Needless to say, technology is getting us closer to depicting and observing every aerodynamic aspect involved in insect flight. Not only will these findings contribute to the knowledge of aerodynamics overall, but they will allow us to diversify insects based on their flight patterns and evolutionary background, and perhaps determine once and for all the evolution of flight. Not only can the aerodynamics of all insects help us to decipher the past, we can also apply it to the future by developing things like robotic models and micro-air vehicles. Understanding how wing-wing interactions affect flight kinematics across different four-winged species of insects will undoubtedly contribute to the overall understanding of aerodynamics as a whole and will allow researchers to optimize lift and minimize drag production in the development of aircrafts, robotics and micro air vehicles. Developing aircrafts with these optimized kinematic conditions will also serve in reducing fuel consumption if applied to the air transport industry.

Understanding the aerodynamics of four-winged insects can contribute to the development of more sophisticated micro-air vehicles. Four-winged insects typically have greater maneuvering capabilities due to the fact that they operate at higher wing beat frequencies. This, for example, would allow a robotic micro-air vehicle to hover, where as a two-winged micro-air vehicle would need to remain in constant forward flight to avoid stall and circle around a fixed location making it difficult to observe a specific area 
of interest. Micro-air vehicles have many possible applications such as search and rescue in major catastrophic events where it may be difficult for personnel to reach.

Pest control is a major issue in the African migratory locusts. They fly in swarms and devastate crops across the continent. Acquiring a better understanding of their flight kinematics and neuromuscular control of flight behaviours may lead to the development of safe chemical pesticides that could manipulate the flight behaviours of the locust avoiding crop destruction by disabling the ability of locusts to form swarms or to fly for long distances.

This study has provided a preliminary understanding of forewing interactions on the hindwing in flying locusts. Further studies using flapping wing models in forward translation is important in understanding the magnitude of these interactions of flying locusts in straight, forward flight. 


\section{Literature}

Alexander, R.M. (2003). Principles of Animal Locomotion. Princeton University Press.

Barass, R. (1974). The Locust A Guide for Laboratory Practical Work. $2^{\text {nd }}$ edn. Redwood Burn Limited, Trowbridge \& Esher

Birch, J.M. \& M.H. Dickinson. (2001). Spanwise flow and the attachment of the leading edge vortex on insect wings. Nature. 412, 729-733

Birch, J.M., W.B. Dickson \& M.H. Dicksinson. (2003). Force production and flow structure of the leading edge vortex on flapping wings at high and low Reynolds numbers. J. Exp. Biol. 207, 1063-1072

Burrows, M. (1996). The Neurobiology of an Insect Brain. Oxford University Press, Oxford, New York \& Tokyo.

Cebulski, S. (2010). Leading edge vortex structure on revolving model wings. M.Sc. Thesis, Carleton University.

Chapman, R.F. (1982). The Insects. Structure and Function, $3^{\text {rd }}$ edn. Hodder and Stoughton, London.

Dawson, J.W., K. Dawson-Scully, D. Robert \& R. M. Robertson. (1997). Forewing asymmetries during auditory avoidance in flying locusts. J. Exp. Biol. 200, 2323 2335

Dawson, J.W., W. Kutsch \& R.M. Robertson. (2004a). Auditory-evoked evasive maneuvers in free-flying locusts and moths. J. Comp. Physiol. A. 190, 69-84

Dawson, J. W., F-H. Leung \& R.M. Robertson. (2004b). Acoustic startle/escape reactions in tethered flying locusts: motor patterns and wing kinematics underlying intentional steering. J. Comp. Physiol. A. 190, 581-600.

Deng, X. \& Z. Hu. (2008). Wing-wing interactions in dragonfly flight. Ins. of Neuromorphic Eng.(INE-WEB.ORG) pg.1-3

Dickinson, M.H., F-O. Lehmann \& S.P. Sane. (1999). Wing rotation and the aerodynamic basis of insect flight. Science. 284, 1954-1960

Dudley, R. (2000). The Biomechanics of Insect Flight. Form, Function, Evolution. Princeton University Press.

Goth, A. (2010). Dorsal wing-flip timing in tethered flying locusts. M.Sc. Thesis, Carleton University. 
Ellington, C.P. (1984). The aerodynamics of flapping animal flight. Amer. Zoologist. 24, 95-105

Ellington, C.P. (1984a). The aerodynamics of hovering insect flight. III. Kinematics. Phil. Tran. R. Soc. Lond. B. 305, 41-78

Ellington, C.P. (1984b). The aerodynamics of hovering insect flight. IV. Aerodynamic mechanisms. Phil. Tran. R. Soc. Lond. B. 305, 79-113

Ellington, C.P., C. van de Berg, A.P. Willmot \& A.L.R. Thomas. (1996). Leading-edge vortices in insect flight. Nature. 384, 626-630

Ellington, C.P. (1999). The novel aerodynamics of insect flight: Application to micro-air vehicles. J. Exp. Biol. 202, 3439-3448

Fauquier, J. (2008). The Effects of a rigid tether vs. a loose tether during an acoustic startle response in locusts (Locusta migratoria). B.Sc. Thesis, Carleton University.

Fry, S.N., P. Müller, H-J. Baumann, A.D. Straw, M. Bichsel \& D. Robert. (2004). Context-dependent stimulus presentation to freely moving animals in 3D. J. Neyr. Meth. 135, 149-157

Hendrick, T. (2008). Software techniques for two- and three-dimensional kinematic measurements of biological and biomimetic systems. Bioinsp. Biomim. 3, 1-6.

Jensen, M. (1956). Biology and physics of locust flight. III. The aerodynamics of locust flight. Phil. Tran. R. Soc. Lond. B. 239, 511-552

Kutsch, W. (1999). Timing of elevator muscle activity during climbing in free locust flight. J. Exp. Biol. 202, 3575-3586

Kammer, A. E. (1971). The motor output during turning flight in a hawkmoth, Manduca sexta. J. Insect Physiol. 17, 1073-1086

Kutsch, W., S. Berger \& H. Kautz. (2003). Turning manoeuvres in free-flying locusts: Two-channel radio-telemetric transmission of muscle activity. J. Exp. Zoology 299A, 139-150

Kutsch, W. \& U. Fuchs. (2000). Locust flight initiation: a comparison of normal and artificial release. Physiol. Ent. 25, 370-382

Lehmann, F-O. \& S. Pick. (2007). The aerodynamic benefit of wing-wing interaction depends on stroke trajectory in flapping insect wings. J. Exp. Biol. 210, 1362-1377 
Lehmann, F-O., S.P. Sane \& M. Dickinson. (2005). The aerodynamic effects on wingwing interaction in flapping insect wings. J. Exp. Biol. 208, 3075-3092

Lester, R. L., C. Grach, M.P. Pener \& S.J. Simpson. (2005). Stimuli inducing gregarious colouration and behaviour in nymphs of Schistocerca gregaria. J. Ins. Physiol. 51, 737-747

Liu, H., C. Ellington, K. Kawachi. (1998). A computational fluid dynamic study of hawkmoth hovering. J. Exp. Biol. 201, 461-477

Lu, Y., G.X. Shen \& G.J. Lai. (2006) Dual leading-edge vorticies on flapping wings. J. Exp. Biol. 209, 5005-5016

Michelson, R.C. \& M.A. Naqvi. (2003). Beyond biologically-inspired insect flight. Low Re aerodynamics on aircraft including applications in emerging UAV technology. RTO-AVT von Karman Institue for Fluid Dynamics.

Murphy. (2005). Fore and hindwing aerodynamic interactions in locust flight. M. Phil. Thesis, Cambridge University

Ramamurti, R. \& W.C. Sanberg. (2002). A three-dimensional computational study of the aerodynamic mechanisms of insect flight. $J$. Exp. Biol. 205, 1507-1518.

Robert, D. (1989). The auditory behaviour of flying locusts. J. Exp. Biol. 147, 279-301

Robertson, R.M. \& A.G. Johnson. (1993). Retinal image size triggers obstacle avoidance in flying locusts. Naturwissenschaften. 80, 176-178

Robertson, R.M., \& D.N. Reye. (1992). Wing movements associated with collisionavoidance maneuvers during flight in the locust Locusta migratoria. J. Exp. Biol. $163,231-258$

Robertson, R.M., C.T. Kunhert \& J.W. Dawson. (1996). Thermal avoidance during flight in the locust Locusta migratoria. J. Exp. Biol. 199, 1383-1393

Roeder, K. D. (1967). Turning tendency of moths exposed to ultrasound while in stationary flight. $J$. Insect Physiol. 13, 873-888

Sane, S.P. (2003). The aerodynamics of insect flight. J. Exp. Biol. 206 (23), 4191-4208

Sane, S.P. \& M.H. Dickinson. (2002). The aerodynamic effects of wing rotation and a revised quasi-steady model of flapping flight. J. Exp. Biol. 205, 1087-1096

Schnackenburg, K., M. Martinez, J. Laliberté \& J. Dawson. (2007) Towards better Artificial Insect Wings for Flow Visualization and Micro-Air Vehicles. ICAST Conference. 
Shoemaker, K.L., \& R.M. Robertson. (1998). Flight motor patterns of locusts responding to thermal stimuli. J. Comp. Physiol. A. 183, 477-488

Smith, C.W., R. Herbert, R.J. Wooton \& K.E. Evans. (2000). The hind wing of the desert locust (Schistocerca gregaria Forskål). II. Mechanical properties and functioning of the membrane. J. Exp. Biol. 203, 2933-2943

Somps, C. \& M Luttges. (1985). Dragonfly flight: Novel uses of unsteady separated flows. Science. 228, 1326-1329

Thomas, A.L.R., G.K. Taylor, R.B. Srygley, R.L. Nudds \& R.J. Bomphrey. (2004). Dragonfly flight: free-flight and tethered flow visualizations reveal a diverse array of unsteady lift-generating mechanisms, controlled primarily via angle of attack. $J$. Exp. Biol. 207, 4299-4323

Usherwood, J.R. \& F-O. Lehmann. (2008). Phasing of dragonfly wings can improve aerodynamic efficiency by removing swirl. $J$. R. Soc. Interface. 5, 1303-1307

Van den Berg, C. \& C.P. Ellington. (1997). The vortex wake of a 'hovering' model hawkmoth. Phil. Tran. R. Soc. Lond. B 352, 317-328

Vogel, S. (2003). Comparative Biomechanics: Life's a Physical World. Princeton University Press.

Wang, Z.J. \& M. Sun. (2005). A computational study of the aerodynamics and forewinghindwing interaction of a model dragonfly in forward flight. J. Exp. Biol. 208, 3785-3804

Wang, Z.J. \& D. Russel. (2007). Effect of forewing and hindwing interactions on aerodynamic forces and power in hovering dragonfly flight. Phys. Rev. Lett. 99, 148101(1)-148101(4)

Weis-Fogh, T. (1956). Biology and physics of locust flight. II flight performance of the desert locust (Schistocerca gregaria). Phil. Trans. R. Soc. Lond. 239, 459-510

Weis-Fogh, T. (1973). Quick estimates of flight fitness in hovering animals, including novel mechanisms for lift production. J. Exp. Biol. 59, 169-230

Wilson, D.M. \& T. Weis-Fogh. (1962). Patterned activity of co-ordinated motor units, studied in flying locusts. J. Exp. Biol. 39, 643-667.

Wooton, R.J., K.E. Evans, R. Herbert \& C.W. Smith. (2000). The hind wing of the desert locust (Schistocerca gregaria Forskål). I. Functinal morphology and mode of operation. J. Exp. Biol. 203, 2921-2931 